\title{
Rheology of frictional grains
}

\author{
Dissertation \\ zur Erlangung des mathematisch-naturwissenschaftlichen Doktorgrades \\ "Doctor rerum naturalium" \\ der Georg-August-Universität Göttingen
}

im Promotionsprogramm ProPhys

der Georg-August University School of Science (GAUSS)

\author{
vorgelegt von \\ Matthias Grob \\ aus Mühlhausen
}

Göttingen, 2016 


\section{Betreuungsausschuss:}

- Prof. Dr. Annette Zippelius, Institut für Theoretische Physik, Georg-August-Universität Göttingen

- Dr. Claus Heussinger, Institut für Theoretische Physik, Georg-August-Universität Göttingen

\section{Mitglieder der Prüfungskommission:}

- Referentin: Prof. Dr. Annette Zippelius, Institut für Theoretische Physik, Georg-August-Universität Göttingen

- Korreferent: Prof. Dr. Reiner Kree, Institut für Theoretische Physik, Georg-August-Universität Göttingen

\section{Weitere Mitglieder der Prüfungskommission:}

- PD Dr. Timo Aspelmeier, Institut für Mathematische Stochastik, Georg-August-Universität Göttingen

- Prof. Dr. Stephan Herminghaus, Dynamik Komplexer Fluide, Max-Planck-Institut für Dynamik und Selbstorganisation

- Dr. Claus Heussinger, Institut für Theoretische Physik, Georg-August-Universität Göttingen

- Prof. Cynthia A. Volkert, PhD, Institut für Materialphysik, Georg-August-Universität Göttingen 


\section{Zusammenfassung}

Diese Arbeit behandelt die Beschreibung des Fließens und des Blockierens von granularer Materie. Granulare Materie kann einen Verfestigungsübergang durchlaufen. Dieser wird Jamming genannt und ist maßgeblich durch vorliegende Spannungen sowie die Packungsdichte der Körner, welche das Granulat bilden, bestimmt. Die Rheologie dichter granularer Medien ist zusätzlich zu Spannung und Packungsdichte stark durch Reibung zwischen den Körnern beeinflusst. Wir zeigen mittels numerischer Simulationen und analytischer Betrachtungen, wie Reibung Jamming qualitativ verändert. Reibungsfreies Jamming ist ein kontinuierlicher Phasenübergang mit einem kritischen Punkt bei verschwindender Spannung. Reibungsbehaftetes Jamming ist ein diskontinuierlicher Phasenübergang mit einem kritischen Punkt bei endlicher Spannung. Der kritische Punkt bei endlicher Spannung führt zu bemerkenswertem Verhalten: Oberhalb der kritischen Packungsdichte gibt es ein Intervall an Packungsdichten, innerhalb dessen große oder kleine Spannungen zum Fließen führen, mittlere Spannungen hingegen führen zum Blockieren des Mediums. Das Fließverhalten nahe Jamming ist stark durch die Systemgröße beeinflusst: Es gibt eine kritische Systemgröße, oberhalb derer zeitabhängiger Fluss entsteht. Dieser zeitabhängige Fluss wird durch die Ausbildung von großskaligen Strukturen im Spannungsfeld erklärt. Sowohl die großskaligen Strukuren als auch der damit einhergehende zeitabhängige Fluss sind neuartige Phänomene im Fluss von trockenen Granulaten und durch Reibung hervorgerufen. 



\section{Abstract}

This thesis deals with the description of flow and arrest of granular matter. Granular matter can undergo a rigidity transition - called jamming - that is mainly controlled by the applied stresses and the packing fraction of the grains that constitute the medium. In addition to stress and packing fraction, interparticle friction greatly affects the rheology of granular matter. Using numerical simulations and analytical modeling, we show how novel behavior in dense flow and jamming regimes arises in the presence of friction. In particular, frictionless jamming is continuous with a critical point at zero stress. In contrast, frictional jamming is shown to exhibit a discontinuous phase transition with a critical point at finite stress. The fact that the critical point resides at finite stress gives rise to remarkable flow behavior, called reentrant flow. Explicitly, there is an interval of packing fractions above the critical packing fraction in which large or low stress leads to flow but intermediate stress jams the medium. The behavior close to jamming depends substantially on the system size, i.e., there is a critical system size above which unsteady flow emerges. Unsteady flow is rationalized by large-scale structures in the stress fields. Both, the large-scale structures and the accompanied unsteady flow, are novel phenomena regarding the flow of dry granular matter and can be attributed to interparticle friction. 



\section{Contents}

\begin{tabular}{ll}
\hline I. Introduction & 1
\end{tabular}

1. An introduction to granular media 3

2. Scope of the thesis 5

$\begin{array}{ll}\text { 3. Models for grains and friction } & \mathbf{7}\end{array}$

3.1. Deformation of frictionless spheres . . . . . . . . . . . 7

3.2. Frictional interactions . . . . . . . . . . . . . . . . . 10

$\begin{array}{ll}4 . \text { The jamming transition } & 13\end{array}$

4.1. Frictionless jamming . . . . . . . . . . . . . . . . . . . 15

4.2. $\quad$ Frictional jamming . . . . . . . . . . . . . . . . . . . . . . . . . 18

$\begin{array}{ll}\text { 5. Shear rheology } & 21\end{array}$

5.1. Rheometry and flow curves - an overview . . . . . . . . . . . 21

5.2. Inertial flow . . . . . . . . . . . . . . . . . 24

5.3. Plastic flow . . . . . . . . . . . . . . . . . . 25

5.4. Shear thickening . . . . . . . . . . . . . . . . . . 27

5.5. Unsteady flow . . . . . . . . . . . . . . . . . . . 30

$\begin{array}{ll}\text { 6. Active microrheology } & 33\end{array}$

$\begin{array}{ll}\text { II. Results } & 35\end{array}$

\begin{tabular}{ll}
\hline 7. Shear rheology of frictional grains & 39
\end{tabular}

7.1. Jamming of frictional particles . . . . . . . . . . . . . . . . 39 
Contents

7.2. Rheological chaos of frictional grains . . . . . . . . . . . . . 46

7.3. Unsteady rheology and heterogeneous flow of dry frictional grains 52

8. Active microrheology of driven granular particles 65

\begin{tabular}{|ll}
\hline III. Summary and discussion & 73
\end{tabular}

\begin{tabular}{|lr}
\hline IV. Outlook & 79
\end{tabular}

$\begin{array}{ll}\text { V. Appendix } & 85\end{array}$

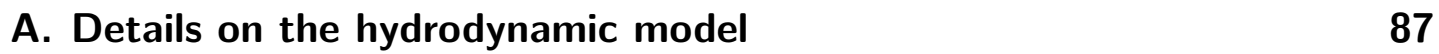

A.1. The flowing state . . . . . . . . . . . . . . . . . . 88

A.2. The shear jammed state . . . . . . . . . . . . . . 89

\begin{tabular}{lr}
\hline Bibliography & 91
\end{tabular}

\begin{tabular}{ll}
\hline Author contribution & 105
\end{tabular}

\begin{tabular}{|l|l}
\hline Acknowledgments & 107
\end{tabular}

\begin{tabular}{lr}
\hline List of Publications & 109
\end{tabular} 
Part I.

\section{Introduction}





\section{An introduction to granular media}

If we measure it by tons, the material most manipulated by man is water; the second-most-manipulated is granular matter.

(de Gennes [1999])

Granular media - collections of grains - are ubiquitous in nature, daily life, and engineering disciplines. Grains constitute the seabed, dunes, beaches, and an important proportion of the earth's soil. We use pepper, sugar, and salt, coffee beans or powder, and many other kinds of granular media in the kitchen and in our daily life. Sand is a resource of which glass, semiconductors, concrete, and ultimately buildings are made. Granular matter as a resource or consumer products is handled in industrial processes, which, in total, are estimated to be responsible for a tenth of the planet's energy budget Mullin, 2002]. The amount of energy for the handling of grains, i.e., for processes where granular media are deformed or transported, highlights the importance of a proper understanding of flow properties of granular matter as a complex fluid.

Grains are composed of a vast number of molecules and the energy associated with motion or contact is by orders of magnitude larger than the thermal energy, $k_{\mathrm{B}} T$. Granular media are therefore considered athermal Bi et al., 2015, Brilliantov and Pöschel, 2010]. Interactions between grains are inelastic, i.e., in a collision, energy is dissipated into internal degrees of freedom, see, e.g., Landau and Lifshitz, 1970]. Furthermore, when grains are in contact, any motion or force parallel to the contact surface is opposed by a frictional force Johnson, 1985]. Energy scale separation, the inelastic character of collisions, and friction make granular media distinct; classical equilibrium thermodynamics and fluid dynamics are not applicable Kadanoff, 1999. 


\section{An introduction to granular media}

Granular media are reminiscent of fluids or solids but exhibit a host of unusual phenomena Jaeger et al., 1996]. In contrast to ordinary (simple) fluids, granular fluids do not possess a constant viscosity, i.e., the ratio between shear stress and strain rate depends on the applied shear. An excessive increase in viscosity can ultimately lead to a transition into a solid disordered state with a finite shear modulus. The transition from fluid to solid is called jamming. The solid state, too, shows extraordinary behavior. Granular particles are not bound to each other by chemical bonds but by confinement in a volume. An infinitesimal extension of the confining volume can lead to the complete loss of a finite shear modulus and the initiation of a flowing state. Rheology studies flow problems of fluids which are non-Newtonian, i.e., fluids that possess a viscosity that is not constant as a function of strain rate or shear stress.

The rheological properties depend on a number of aspects and parameters: the packing fraction of the particles, the particles themselves and their interaction, the geometry and boundaries of the problem, and the preparation history. In this thesis, we study these aspects, with a particular focus on the exceptional role played by friction, in a granular medium under shear. 


\section{Scope of the thesis}

In the course of this thesis we deal with dense granular media under shear. In part 1 , we give an overview of the subject. In Chapter 3, we deal with molecular models of granular particles. Chapter 4 focuses on assemblies of grains and explains the jamming transition: a rigidity transition from a flowing state to a disordered solid state. Subsequently, Chapter 5, deals with the shear flow of densely packed particles including phenomena like shear thickening and flow heterogeneities. Chapter 6 describes an approach to probe a complex fluid by the dynamics of a probe particle.

In part II, we present the results of the thesis. This part is divided in two Chapters. Chapter 7 constitutes the main results of this thesis and we investigate the rheology of frictional grains. This Chapter is divided in three sections corresponding to an article each. First, in section 7.1, we discuss the jamming transition of frictional grains in small simulation cells. Results on large systems, in which an unsteady and chaotic response emerges, are presented in section 7.2. There, we also present a simple analytical theory, which is based on coupling of hydrodynamics to a microstructure variable associated with friction and which explains the main features of our simulations. Lastly, we present results that characterize heterogeneous and unsteady flow states and connect the preceding studies in section 7.3 . Chapter 8 covers a microrheology study in a dense granular medium. The nonlinear velocity-force relations of a probe particle are investigated.

Part III summarizes, discusses, and connects the results with a broader context. Part IV] is an outlook to future directions of research on flow of frictional grains. 



\section{Models for grains and friction}

The interaction between grains is fundamental for the phenomena, which we associate with a complex fluid, including the possibility to jam into a solid state. Grains are solid bodies and the description of the dynamics of grains in contact is a formidable task. A contact force, $\mathbf{f}_{i j}$, between granular particles, $i$ and $j$, of mass, $m$, gives rise to motion according to Newton's equation of motion:

$$
m \ddot{\mathbf{x}}_{i}=\sum_{j \neq i} \mathbf{f}_{i j}
$$

with the coordinate, $\mathbf{x}_{i}$, of particle, $i$. The contact forces are governed by the dynamics of the contact and the material properties of the particles. A thorough treatment of contact mechanics including a discussion of friction is given by Johnson 1985. Results relevant for numerical simulations are presented in Schäfer et al. 1996.

First, we give an overview of interactions of frictionless spheres in section 3.1 . Frictionless spheres allow for an extensive theoretical description and a comprehensive understanding of interaction mechanisms. Second, we highlight the importance of incorporating frictional interactions in particle models and present an approach to model friction in section 3.2 .

\subsection{Deformation of frictionless spheres}

In this thesis, we examine the dynamics of assemblies of spherical grains with numerical methods which allow for detailed resolution of positions, contacts, velocities, interparticle forces, and local stresses. In practice, spherical particles possess the advantage that a quantitative study of stress transmission is easily accessible. In laboratory experiments, disks or spheres made from photoelastic materials are used. Stress induced fringe patterns allow for direct inference of 


\section{Models for grains and friction}

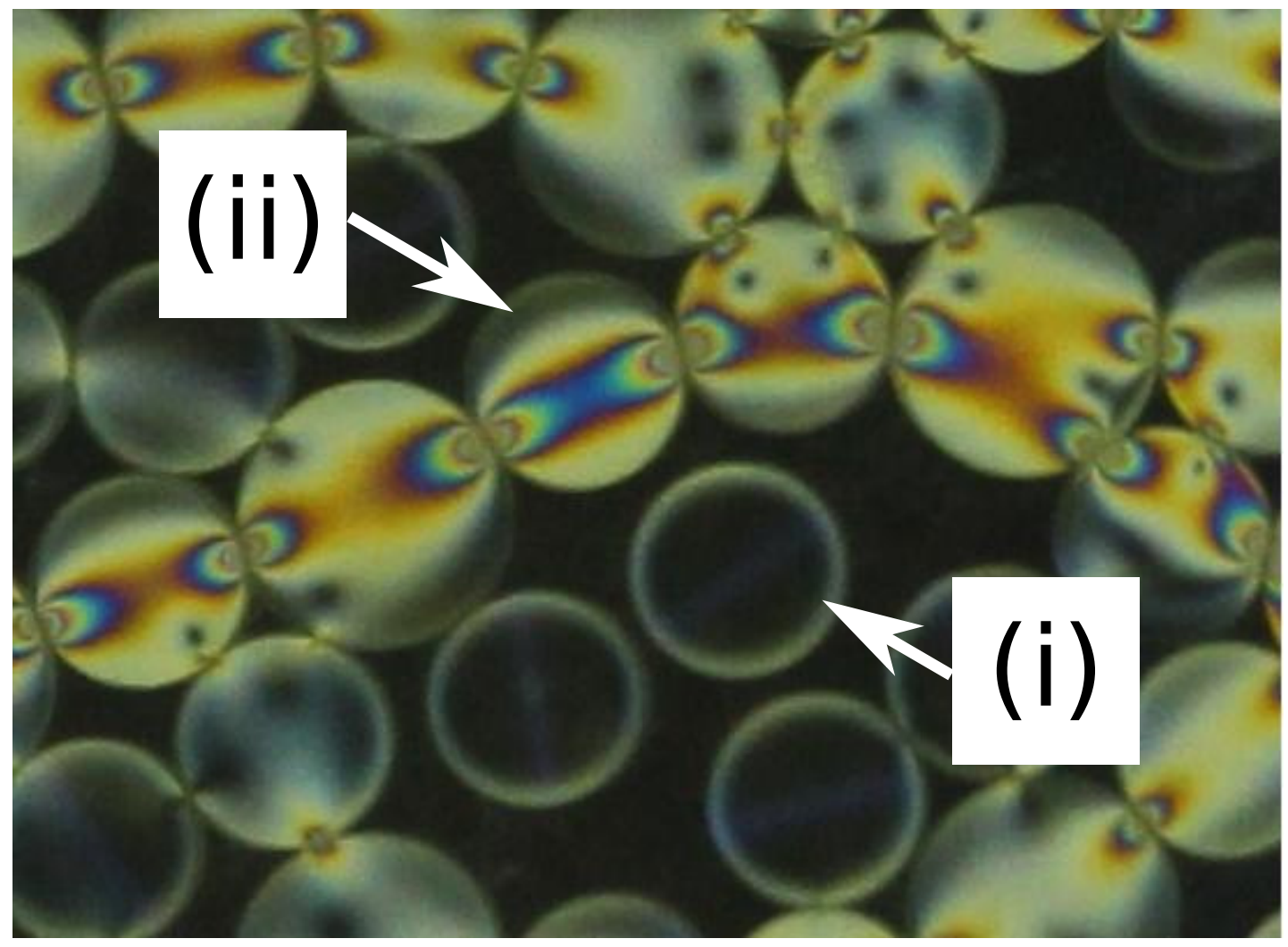

Figure 3.1.: Photograph of photoelastic disks: (i) disk without fringe pattern and (ii) disk with stress originating at the contacts and leading to the fringe pattern. Disks made by Jonathan Barès, Department of Physics \& Center for Nonlinear and Complex Systems, Duke University, Durham, North Carolina 27708, USA. Photo courtesy of J. Barès.

contact points and loads Johnson, 1985. In figure 3.1, we show an assembly of photoelastic disks. Disk (i) is not under stress while contacts on disk (ii) lead to a characteristic fringe pattern.

Ideal frictionless spheres are probably the simplest form of granular grains. Frictionless spheres in contact transmit forces only along the line connecting the spheres' centers, called the normal of a contact. Here, we consider contact interactions only, i.e., no electrostatic interactions, etc. When particles touch just slightly they deform quasistatically like an elastic solid that stores energy in the deformation. The elastic nature of the contact aims at minimizing the stored energy which results in a repulsive force. The elastic energy depends on material parameters and the geometry of the spheres: the Young's modulus, the Poisson 


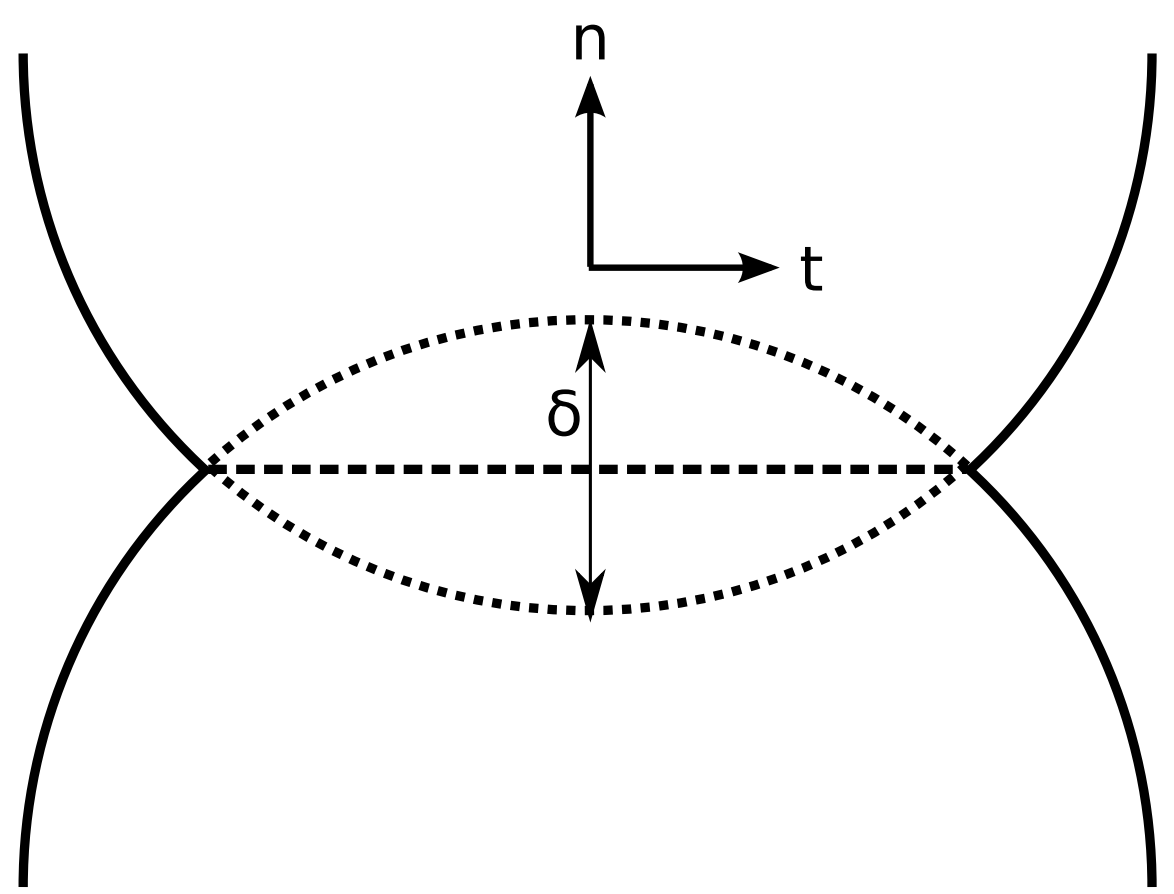

Figure 3.2.: Amount of deformation of particles near the contact surface (dashed line) modeled by the overlap $\delta$. The unit vectors, $\mathbf{n}$ and $\mathbf{t}$, define normal and tangential directions of contact, respectively.

ratio, and the radii of the particles. Frictionless elastic spheres in contact are studied in detail in Hertz 1882; Landau and Lifshitz 1970].

In fact not all the energy is stored as potential energy in the contact. Instead, energy is irreversibly dissipated into the particles when plastic yield occurs or vibrational modes are excited, which we might hear when particles interact (see Michlmayr et al. 2012 for a review). Energy dissipation implies that the impact velocity, $v^{\prime}$, is larger than the post-collisional velocity, $v$. The negative ratio between the velocities after and prior the contact, is called coefficient of restitution:

$$
\epsilon=-\frac{v}{v^{\prime}}
$$

In general, the coefficient of restitution depends on material parameters and on the relative velocity when particles collide [Güttler et al. 2012].

The simplest model that incorporates elastic repulsion and viscous damping due to energy dissipation is the linear spring-dashpot model [Schwager and Pöschel, 2007]. The deformation, or displacement, of particles in contact is described by the overlap, $\delta$, see figure 3.2. The contact dynamics of two spheres in contact 


\section{Models for grains and friction}

with effective mass, $m_{\text {eff }}$, follows a damped harmonic oscillator, where the normal force, $\mathbf{f}^{(\mathrm{n})}$, acts on the displacement along the normal direction, $\mathbf{n}$ :

$$
\mathbf{f}^{(\mathrm{n})}=\left(-k^{(\mathrm{n})} \delta-\eta^{(\mathrm{n})} / m_{\mathrm{eff}} \dot{\delta}\right) \mathbf{n} .
$$

The repulsive and dissipative component is governed by a spring constant, $k^{(\mathrm{n})}$, and a viscous damping constant, $\eta^{(\mathrm{n})}$, respectively. Analogous to Hooke's law, the repulsive component of the force depends linearly on the amount of deformation. The dissipative component is linear with the normal component of the relative velocity of the particles, $\dot{\delta}$. In this particular model, one can show that the coefficient of restitution and the binary collision tim $t_{\mathrm{n}}$, are independent of the impact velocity. In principle, $k^{(\mathrm{n})}$ and $\eta^{(\mathrm{n})}$ can be tuned to meet experimental values for $\epsilon$ and $t_{\mathrm{n}}$ which determine the nature of the collision completely. This simple model lacks the velocity dependent coefficient of restitution and a realistic repulsion law: spherical particles in three dimensions repel with $\mathbf{f}^{(\mathrm{n})} \propto \delta^{3 / 2}$, see Hertz, 1882, and two-dimensional disks repel with more complicated laws, see Gerl and Zippelius, 1999]. However, the linear spring-dashpot model proofed useful and for the sake of simplicity it is widely applied in simulations and theory.

\subsection{Frictional interactions}

In experiment and nature, grains are bodies with rough surfaces. The roughness implies the existence of frictional contact forces: forces which act orthogonal to normal, i.e., tangentially. Experiments on a variety of particulate media, not only granular grains, highlight the role played by friction. Particles are as diverse as PMMA spheres Guy et al. 2015, Pan et al., 2015, corn starch Fall et al., 2015; Jiang et al., 2015], silica spheres Royer et al., 2016], or photoelastic disks Bi et al. 2011. A review on rheology with aspects on friction is given by Denn and Morris, 2014.

Two particles in contact share a common area where the normal forces operate (see figure 3.2). When particles are frictional, rotation or tangential relative motion create tangential stress. Tangential stress results in a tangential force,

\footnotetext{
${ }^{1}$ The binary collision time is defined as the duration of a contact of two particles. In general, the duration of an interaction of more than two particles deviates from the binary collision time.
} 


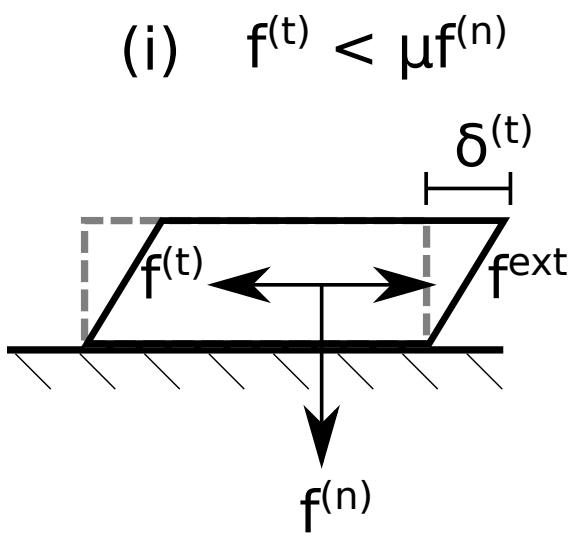

(ii) $f^{(t)}=\mu f^{(n)}$

Figure 3.3.: Sliding block model to illustrate Coulomb's law of friction. The mass of the block gives rise to a normal force, $f^{(n)}$. Left: The block does not slide and the external force, $f^{\text {ext }}$, acting on the block is balanced by the frictional force with the surface, $f^{(\mathrm{t})}$. Due to $f^{\text {ext }}$, the block deforms by $\delta^{(\mathrm{t})}=k^{-1} f$ ext . Right: The block slides and the frictional force cannot balance the external force. In this depiction, $\mu=1$.

$\mathbf{f}^{(\mathrm{t})}$, acting along the tangential of the contact, $\mathbf{t}$, and acting as a resistive force against the motion. A particle, $i$, with moment of inertia, $I$, and angular velocity, $\boldsymbol{\omega}_{i}$, experiences torques due to frictional contacts with particles, $j$ :

$$
I \dot{\boldsymbol{\omega}}_{i}=\sum_{i \neq j} \mathbf{f}_{i j}^{(\mathrm{t})} \times \mathbf{R}_{i j}
$$

with $\mathbf{R}_{i j}$ pointing from particle $i$ 's center to the contact with particle $j$.

The tangential force is bound by the normal force via Coulomb's law of friction, i.e., $\mathbf{f}^{(\mathrm{t})}$ cannot exceed a material specific multiple of the normal force:

$$
\left|\mathbf{f}^{(\mathrm{t})}\right| \leq \mu\left|\mathbf{f}^{(\mathrm{n})}\right|
$$

The Coulomb friction coefficient, $\mu$, is a material parameter. In a frictional contact with $\left|\mathbf{f}^{(\mathrm{t})}\right|=\mu\left|\mathbf{f}^{(\mathrm{n})}\right|$, interfacial slip occurs and the contact is said to slide. Otherwise the contact is called non-sliding. For a pictorial description of Coulomb's law of friction, see figure 3.3 . In general, one discriminates the between a static friction coefficient, $\mu_{\mathrm{s}}$, when the contact is non-sliding and a dynamic friction coefficient, $\mu_{\mathrm{d}}$, when the contact slides. Typically, both parameters are of the same order of magnitude Israelachvili, 2010. Here, we set them equal: $\mu=\mu_{\mathrm{s}}=\mu_{\mathrm{d}}$. 


\section{Models for grains and friction}

For numerical simulations of frictional grains, the model most referenced, that obeys Coulomb's law of friction, was proposed by Cundall and Strack 1979. In the original model, non-sliding contacts store elastic energy according to the tangential displacement, $\delta^{(\mathrm{t})}$. Analogously to the normal interaction, we extend the original model by a dissipative contribution related to the relative tangential velocity at the contact, $\dot{\delta}^{(\mathrm{t})}$. A spring constant, $k^{(\mathrm{t})}$, and a viscous damping constant, $\eta^{(\mathrm{t})}$, determine the mechanical properties. Explicitly, if $\left|\mathbf{f}^{(\mathrm{t})}\right|<\mu\left|\mathbf{f}^{(\mathrm{n})}\right|$ :

$$
\mathbf{f}^{(\mathrm{t})}=\left(-k^{(\mathrm{t})} \delta^{(\mathrm{t})}-\eta^{(\mathrm{t})} \dot{\delta}^{(\mathrm{t})}\right) \mathbf{t} .
$$

If equality holds in Coulomb's criterion and the contact slides, we set $\delta^{(\mathrm{t})}=$ $\mu / k^{(\mathrm{t})}\left|\mathbf{f}^{(\mathrm{n})}\right|$ and $\left|\mathbf{f}^{(\mathrm{t})}\right|=k^{(\mathrm{t})} \delta^{(\mathrm{t})}$ to satisfy Coulomb's criterion ${ }^{2}$. The model was inspired by studies of frictional contacts Mindlin and Deresiewicz, 1953 and used in several rheology studies, see, e.g., Silbert et al., 2001; Otsuki and Hayakawa, 2011; Chialvo et al., 2012; DeGiuli et al., 2015, Henkes et al., 2016]. The presented approach has physical flaws, see Etsion 2010, but its advantages are numerical efficiency and conceptual simplicity. In this thesis, we consider large assemblies of particles and we do not focus on details of collision mechanics and assume that these details are not relevant for the rheology.

\footnotetext{
${ }^{2}$ It would require a study of a particular material to justify this sliding mechanism. In the literature, a number of models exist and there is no obvious consensus which is justified by a (simple) physical argument.
} 


\section{The jamming transition}

As illustrated in figure 4.1, sparsely distributed particles in a finite volume can move freely over a finite distance, i.e., without the interaction with other particles. Densely packed grains may be blocked by contacts with their neighbors and not even an infinitesimal motion is possible. This geometric statement has important

(i)

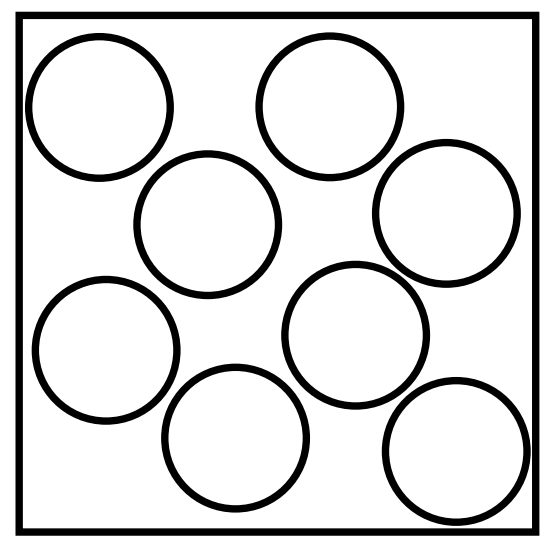

(ii)

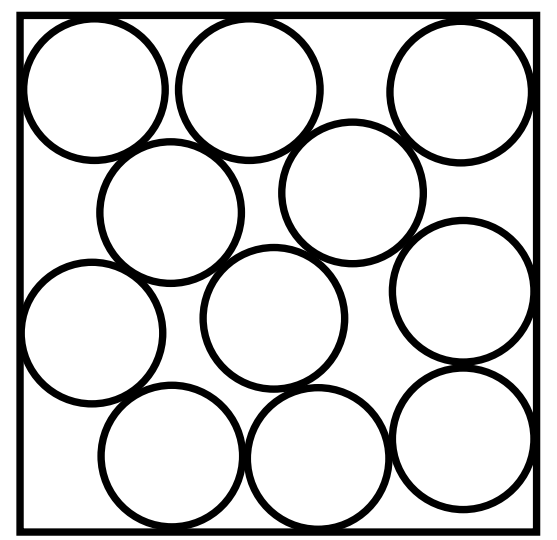

Figure 4.1.: Particles in (i) are not geometrically constrained and can move without the expense of energy. In (ii), particles are densely packed and any motion results in repulsive forces exerted by the particles at contact.

implications for the mechanical behavior of the whole assembly: Volume (i) can be sheared with any shear stress but volume (ii) can sustain a finite maximum shear stress - called yield stress - without deforming. A parameter to describe the geometrical properties of an assembly of $N$ grains with volumes $V_{i}$ confined in a volume $V$ is the packing fraction:

$$
\phi=\frac{\sum_{i=1}^{N} V_{i}}{V}
$$




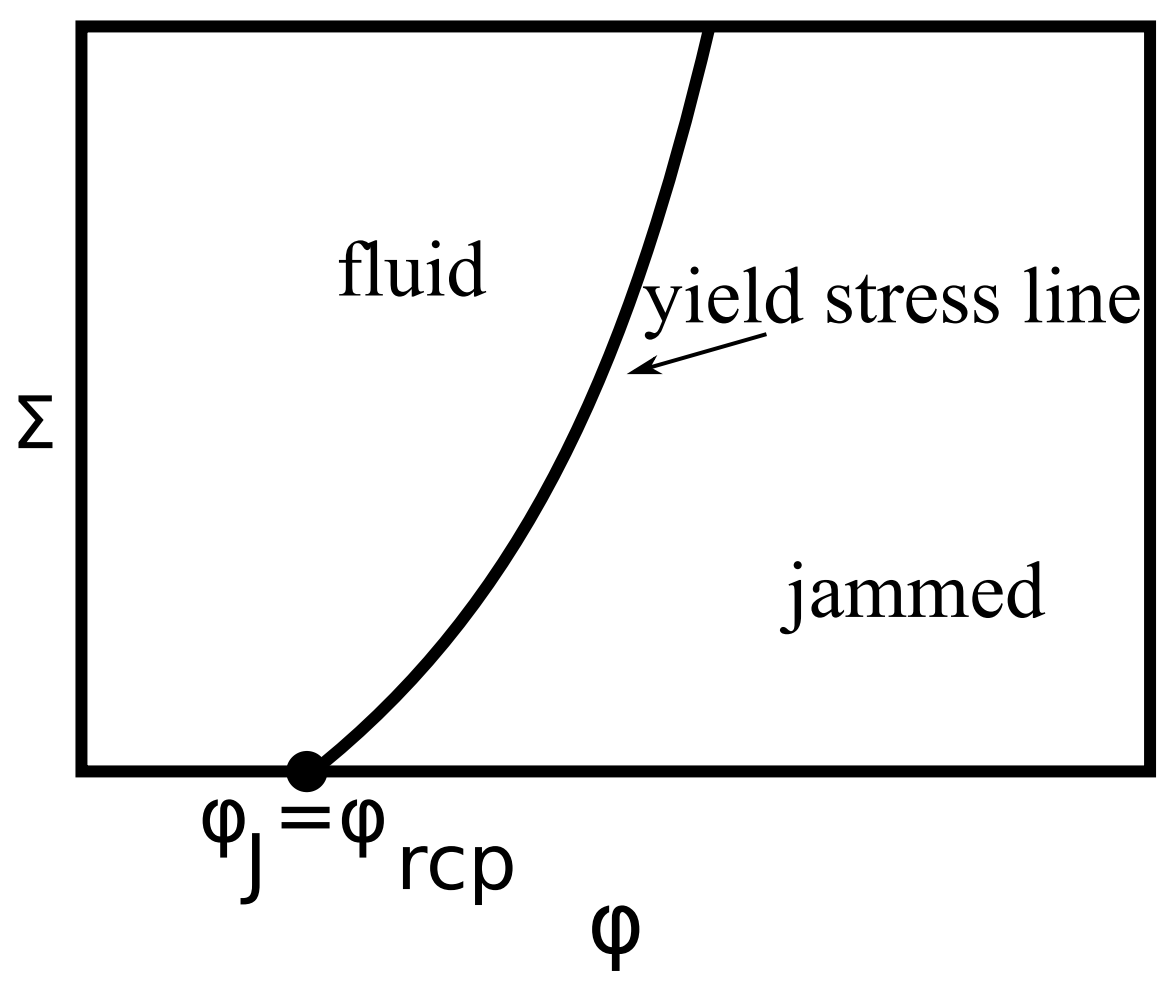

Figure 4.2.: Schematic jamming phase diagram for frictionless spheres. The phase and thus the mechanical response is determined by the shear stress, $\Sigma$, and the packing fraction, $\phi . \phi_{\mathrm{J}}$ is the onset of rigidity at zero stress, $\Sigma=0$.

When the packing fraction increases, the granular medium can jam from a flowing state, as in figure 4.1 (i), into an unordered solid state, as in figure 4.1(ii). Figure 4.2 shows a schematic phase diagram for the system in figure 4.1. similar to the athermal plane in Liu and Nagel [1998]. The shear stress, $\Sigma$, and the packing fraction, $\phi$, control which state is realized: fluid or jammed. A single line, the yield stress line, separates regions of flowing states from jammed states. The yield stress line touches zero stress, $\Sigma=0$, at the jamming packing fraction, $\phi_{\mathrm{J}}$, which sets the jamming point, called point $J$.

The phase diagram in figure 4.2 serves as a high-level description of the jamming transition for a broad range of particulate media. However, the jamming transition cannot be controlled by packing fraction and stress only, but is also influenced by the preparation protocol and the mechanical properties of the particles. We take into account the particles' roughness by considering frictional contacts, as described in Chapter 3 . 
In section 4.1, we discuss the basic properties of the jamming transition and focus on experiments and simulations of frictionless particles: 1 . Subsequently, we deal with jamming of frictional particles in section 4.2, where we discuss experimental and numerical results that are directly linked to frictional interactions.

\subsection{Frictionless jamming}

In this section, we discuss results on the jamming transition of frictionless spheres and rheological properties in the vicinity of the jamming transition.

A fixed volume that is densely and randomly packed with hard spheres, see fig. 4.1(ii), cannot be deformed: The configuration is jammed. The geometric study of such packings of spheres shows that there is a largest packing fraction of randomly packed (hard) spheres, namely the random close packing, $\phi_{\text {rcp }}$ Song et al. 2008. The jamming packing fraction approaches random close packing in the thermodynamic limit O'Hern et al. 2003:

$$
\phi_{\mathrm{J}} \rightarrow \phi_{\mathrm{rcp}} \quad \text { for } \quad N \rightarrow \infty \quad \text { with } \quad \phi=\text { const. }
$$

The jamming transition depends on the preparation of the jammed configuration, see e.g., Chaudhuri et al., 2010. In particular in a finite system, $\phi_{\mathrm{J}}$ depends on the initial condition and is localized in a finite interval, which narrows around $\phi_{\text {rcp }}$ when the system size increases O'Hern et al. 2002. For systems of frictionless particles in two dimensions, the point $J$ is accurately determined by simulations of overdamped, bidisperse, and soft particles in simple shear geometry at zero stress and packing fraction Olsson and Teitel, 2011]:

$$
\phi_{\mathrm{J}}=0.84347 \pm 0.00020
$$

Even though this value changes with the protocol, equation 4.3 gives a robust estimate for jamming in two dimensions. Depending on the protocol, Chaudhuri et al. [2010] observed a range of packing fractions, $\left(\phi_{\mathrm{l}}, \phi_{\mathrm{u}}\right)$, in which jamming is possible with a relative size of $\left(\phi_{\mathrm{u}}-\phi_{\mathrm{l}}\right) / \phi_{\mathrm{u}} \approx 0.023 \%$ in the thermodynamic limit in three dimensions.

\footnotetext{
${ }^{1}$ Friction cannot be switched off in experiments. However, experiments which study universal properties of the jamming transition are also discussed in section 4.1
} 


\section{The jamming transition}

The jamming transition has been investigated thoroughly and identified as a second-order, i.e., continuous, phase transition. The extraction of critical exponents by numerical simulations confirms a continuous jamming scenario with a zero-stress critical point, e.g., Olsson and Teitel [2007]; Otsuki and Hayakawa 2008]; Heussinger and Barrat [2009]; Otsuki and Hayakawa 2009]; Otsuki et al. 2010]; Hatano [2010]; Heussinger et al. [2010]; Vågberg et al. [2011].

The macroscopic mechanical properties of a granular assembly are directly linked to the jamming point. At a packing fraction, $\phi_{\mathrm{J}}$, a rigidity transition takes place and we expect solid like behavior above $\phi_{\mathrm{J}}$, i.e., finite and positive shear and bulk moduli. Indeed, in an isotropic system, the packing fractions where shear and bulk moduli become nonzero equal $\phi_{\mathrm{J}}$ O'Hern et al. [2003]. $\phi_{\mathrm{J}}$ is considered the critical packing fraction for the onset of rigidity, with a yield stress, $\Sigma_{\mathrm{Y}}$, that vanishes at point J. Below $\phi_{\mathrm{J}}$, only fluid states can exist and the stress is determined by the strain rate, $\dot{\gamma}$, and a viscosity, $\eta$, that depends on the packing fraction ${ }^{2}$.

In the vicinity of $\phi_{\mathrm{J}}$, both states, solid and fluid, obey scaling laws with distance to $\phi_{\mathrm{J}}$. Guided by numerical simulations, critical exponents for the behavior of yield stress, viscosity, and stress are determined Otsuki and Hayakawa, 2008, 2009; Otsuki et al., 2010, Hatano, 2010. For a comprehensive review on scaling exponents, see Dinkgreve et al., 2015. Here, we state the exponents for soft dry granular particles with inertia in two dimensions. In the solid state above jamming, $\phi>\phi_{\mathrm{J}}$, a finite yield stress emerges with distance to jamming:

$$
\Sigma_{\mathrm{Y}} \propto\left(\phi-\phi_{\mathrm{J}}\right)^{\beta}
$$

with an exponent, $\beta=1$. In the fluid state, $\phi<\phi_{\mathrm{J}}$, the viscosity, $\eta$, diverges with the distance to jamming in the limit of zero strain rate:

$$
\eta \propto\left(\phi_{\mathrm{J}}-\phi\right)^{-\alpha}
$$

with an exponent, $\alpha=4$. At the critical packing fraction, $\phi=\phi_{\mathrm{J}}$, the shear

\footnotetext{
${ }^{2}$ In a Newtonian fluid, the viscosity is defined by the relation $\Sigma=\eta \dot{\gamma}$. In a dry granular medium, there is no linear dependence between shear stress and strain rate and the socalled generalized viscosity is defined by $\Sigma=\eta \dot{\gamma}^{2}$, see section 5.1 for further information.
} 
stress depends on the strain rate and scales as:

$$
\Sigma\left(\phi_{\mathrm{J}}\right) \propto \dot{\gamma}^{\chi}
$$

with an exponent, $\chi=2 / 5$.

The rheological properties close to the jamming transition are related to the particles and their motion. While approaching the jamming transition, particles tend to move cooperatively when the granular medium is sheared: The motion of one particle induces motion of other particles. This cooperative motion is rationalized by a length scale, $\xi$, which diverges at the jamming transition:

$$
\xi \propto\left|\phi-\phi_{\mathrm{J}}\right|^{-\nu}
$$

with an exponent, $\nu$, typically estimated as $\nu \in[0.1,1]$ and possibly different above and below jamming; For different estimates see, e.g, Lechenault et al. 2008; Heussinger and Barrat, 2009; Vågberg et al., 2011; Liu et al., 2014; Kawasaki et al. 2015]. The diverging length scale near the transition from arrest to flow is universal. A diverging length scale near jamming is observed for a variety of systems: Flows down an inclin€ ${ }^{3}$ e.g., Pouliquen, 2004, Baran et al., 2006; Bonnoit et al., 2010], simple shear geometry, e.g., Olsson and Teitel, 2007; Goyon et al., 2008; Heussinger and Barrat, 2009; Lemaître and Caroli, 2009; Liu et al., 2014, Poiseuille flow, e.g., Goyon et al., 2008; Tewari et al., 2009, or a vibrated monolayer of brass cylinders, e.g., Lechenault et al. 2008.

The existence of a length scale points towards finite size effects. Finite size effects are predicted by theory [Bocquet et al., 2009] and observed in experiments Goyon et al. 2008. Systematic studies of finite size effects to gain insights in the jamming transition are done numerically, e.g., Goodrich et al. 2012, Liu et al. 2014; Vågberg et al., 2014, Goodrich et al., 2014. In Kawasaki et al. 2015], the authors argue for possible finite size effects when the length scale becomes comparable to the linear extension of the system.

\footnotetext{
${ }^{3}$ In this example the packing fraction is not a control parameter. The angle of the incline can control whether flow or arrest of a deposited material is achieved.
} 


\section{The jamming transition}

\subsection{Frictional jamming}

Friction changes the topology of the jamming phase diagram fundamentally. A theoretical description of frictional hard spheres shows that jamming occurs in a range of $\phi_{\text {rlp }}$ up to $\phi_{\text {rcp }}$, where $\phi_{\text {rlp }}$ is called random loose packing Song et al., 2008. In two dimensions, $\phi_{\text {rlp }} \approx 0.767$ Silbert, 2010. The range from $\phi_{\text {rcp }}$ up to $\phi_{\text {rlp }}$ is considerably larger $(\approx 1 \%)$ than what can be produced for frictionless particles by changes in the protocol, see section 4.1. While $\phi_{\text {rlp }}$ is the lower bound for infinite friction coefficient, $\phi_{\mathrm{rcp}}$ is the upper bound that is approached by $\phi_{\mathrm{J}}$ in the frictionless limit. That is, the jamming packing fraction is a function of the friction coefficient, $\phi_{\mathrm{J}}=\phi_{\mathrm{J}}(\mu)$. Thus, friction opens a wide room for jamming to take place.

Studies with photoelastic disks under external stress, see Bi et al., 2011, show that friction allows for anisotropic jammed states: States where stress transmission is anisotropic, which implies the expectation of a rigid response along restricted directions but fluid response in others [Cates et al. 1998]. Anisotropic jammed states are observed at packing fractions lower than $\phi_{\mathrm{J}}$, which is the isotropic jamming packing fraction. The anisotropic jammed states are called shear jammed and are observed above a packing fraction $\phi_{\mathrm{SJ}}<\phi_{\mathrm{J}}$. At $\phi_{\mathrm{SJ}}$, the yield stress of the jammed state is finite and vanishes discontinuously upon decreasing the packing fraction. For a schematic phase diagram for the frictional jamming scenario inferred from Bi et al. [2011], see figure 4.3. Shear jamming is also found in corn starch experiments Fall et al., 2015, Jiang et al., 2015 and rheometry with PMMA spheres confirms the extraordinary impact of frictional contacts Guy et al. 2015; Pan et al. 2015.

Friction enriches rheological properties close to jamming by additional dynamical phases of transient flow when granular media are sheared Ciamarra et al., 2011. In contrast to frictionless systems, frictional systems show clear firstorder like phenomena like discontinuous flow curves and hysteresis Otsuki and Hayakawa, 2011. Moreover, the authors showed that $\phi_{\mathrm{J}}$ loses its exceptional meaning to characterize scaling relations. The scaling exponents are not affected by friction but $\phi_{\mathrm{J}}$ alone does not suffice to characterize the scaling of the divergence of the viscosity and of the yield stress. 


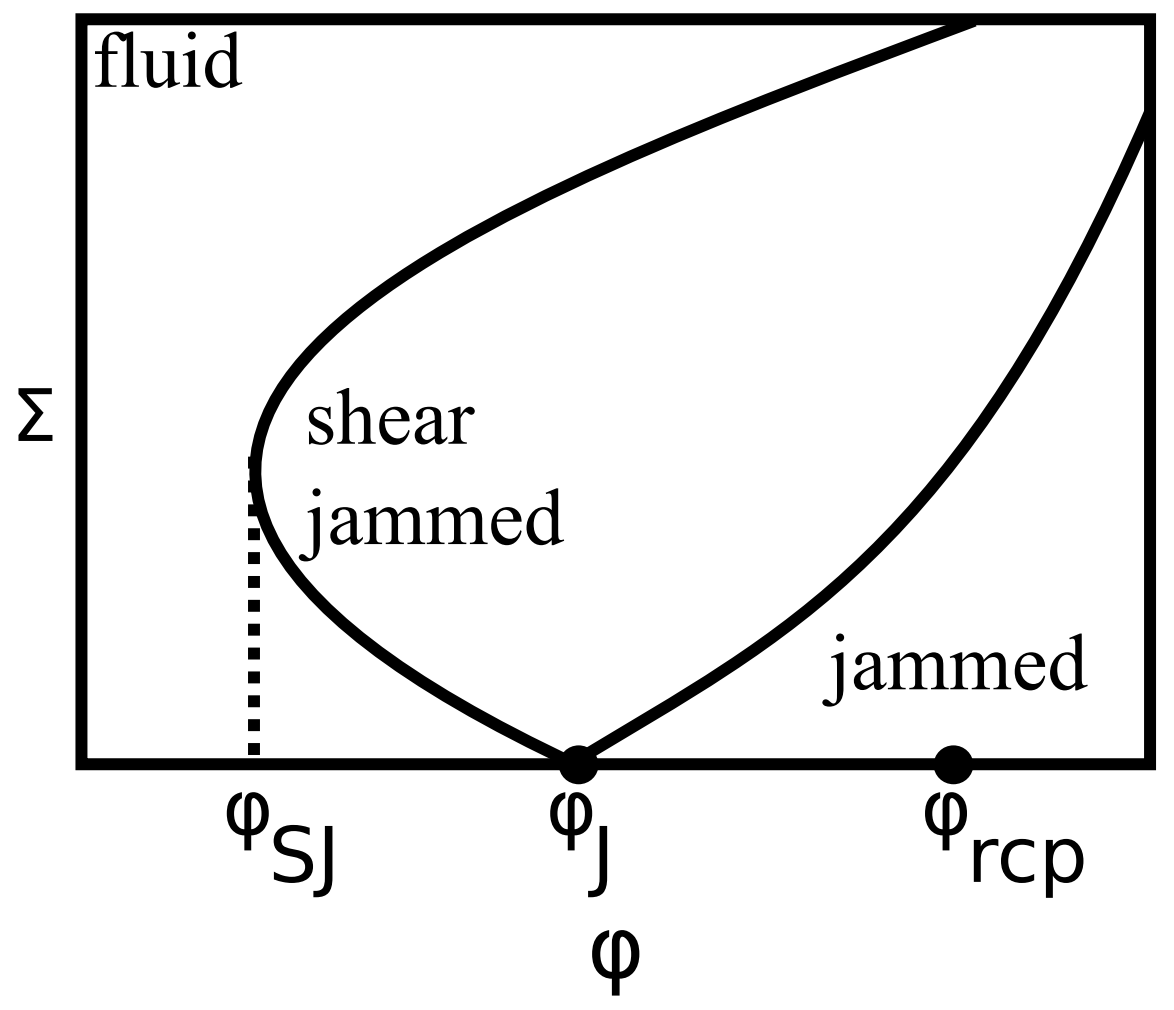

Figure 4.3.: Schematic jamming phase diagram for frictional particles following $B$ et al. 2011. Anisotropic shear jammed states exist below $\phi_{\mathrm{J}}<\phi_{\mathrm{rcp}}$. Jammed states with the lowest packing fraction possible, $\phi_{\mathrm{SJ}}$, possess a finite yield stress, $\Sigma_{\mathrm{Y}} \neq 0$. 



\section{Shear rheology}

This Chapter deals with the rheological investigation of dense granular matter. In section 5.1, we explain how rheological data is acquired experimentally. We give an overview of the possible outcomes, which are collected in flow curves that describe the relation between shear stress and strain rate. Complex fluids exhibit different flow regimes, which are distinguished by the relation between stress and strain rate. In sections 5.2 and 5.3 , we deal with flow regimes of granular media. A transition between these flow regimes, called shear thickening, is treated in section 5.4. Close to transitions, unsteady flow emerges, which is discussed in section 5.5 .

\subsection{Rheometry and flow curves - an overview}

The mechanical response to strain or stress in the proximity of the jamming transition is probed in rheometers or with computer simulations. The measured response characterizes flow properties and provides insight into the jamming transition itself. A rheometer is sketched in figure 5.1. The probe is confined in a fixed volume by a pair of plates ${ }^{1}$. Hence, the packing fraction of the particles in the volume is a control parameter. One confining plate remains fixed while the other plate rotates with either constant angular velocity (constant strain rate) or constant torque (constant shear stress). The complementary quantity is measured in terms of the response of the medium, which is characteristic of the flow regime.

Typical flow curves of frictionless granular media with different packing fractions are shown in figure 5.2 (left). When the probe is dense, athermal, dry, and composed of soft particles three different flow regimes are identified Campbell,

\footnotetext{
${ }^{1}$ There are also rheometers that guarantee fixed normal load. Then the volume of the probe changes in course of the experiment.
} 


\section{Shear rheology}

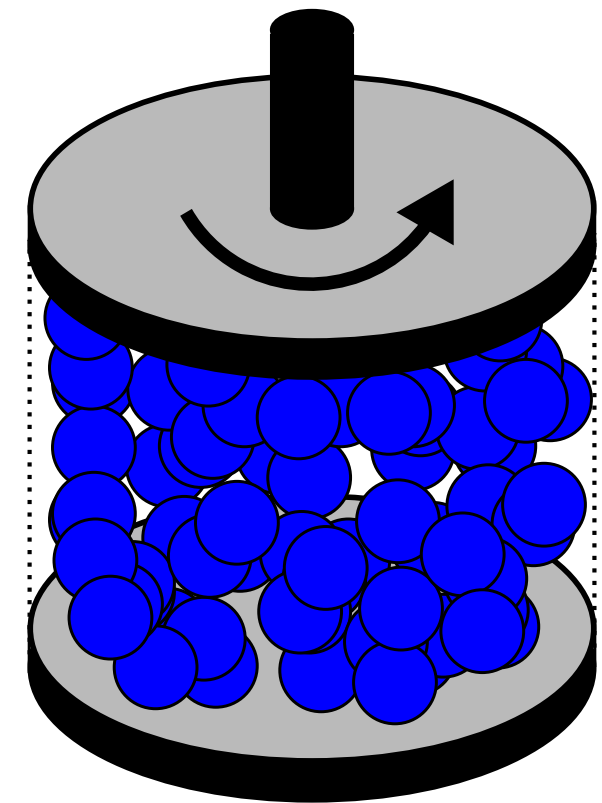

Figure 5.1.: Sketch of a rheometer. A fixed volume between plates (closed by dotted lines) is filled with particles (blue). The lower plate remains at rest and the upper plate rotates with constant angular velocity or torque (indicated by arrow).

\section{2; Chialvo et al., 2012:}

(i) Inertial or Bagnold flou $\left.\right|^{2}$ at low strain rate and low packing fraction, $\phi<$ $\phi_{J}$

(ii) quasistatic flow at low strain rate and densely packed media, $\phi>\phi_{J}$, and

(iii) elastic-inertial flow at large strain rate.

Both, quasistatic and elastic-inertial flow, are referred to as plastic flow.

The flow curves can be utilized to characterize the jamming transition. The scaling laws of yield stress and viscosity, which are discussed in section 4.1, allow for a determination of the jamming packing fraction and scaling exponents. Above $\phi_{\mathrm{J}}$, in the quasistatic regime, see 5.2 (left, ii), the stress plateau at low strain rate equals the yield stress. The yield stress vanishes at $\phi_{\mathrm{J}}$ and grows above. Figure 5.2 (right) shows the generalized viscosity, $\eta=\Sigma / \dot{\gamma}^{2}$. Below $\phi_{\mathrm{J}}$, in

\footnotetext{
${ }^{2}$ The interaction and dissipation mechanisms (e.g., drag forces, friction, etc.) influence the rheological response and determine the flow curve Vågberg et al. 2014. As a consequence, e.g., when the grains are suspended in a Newtonian fluid, a Newtonian regime, i.e., $\Sigma \propto \dot{\gamma}$, appears instead of Bagnold flow.
} 

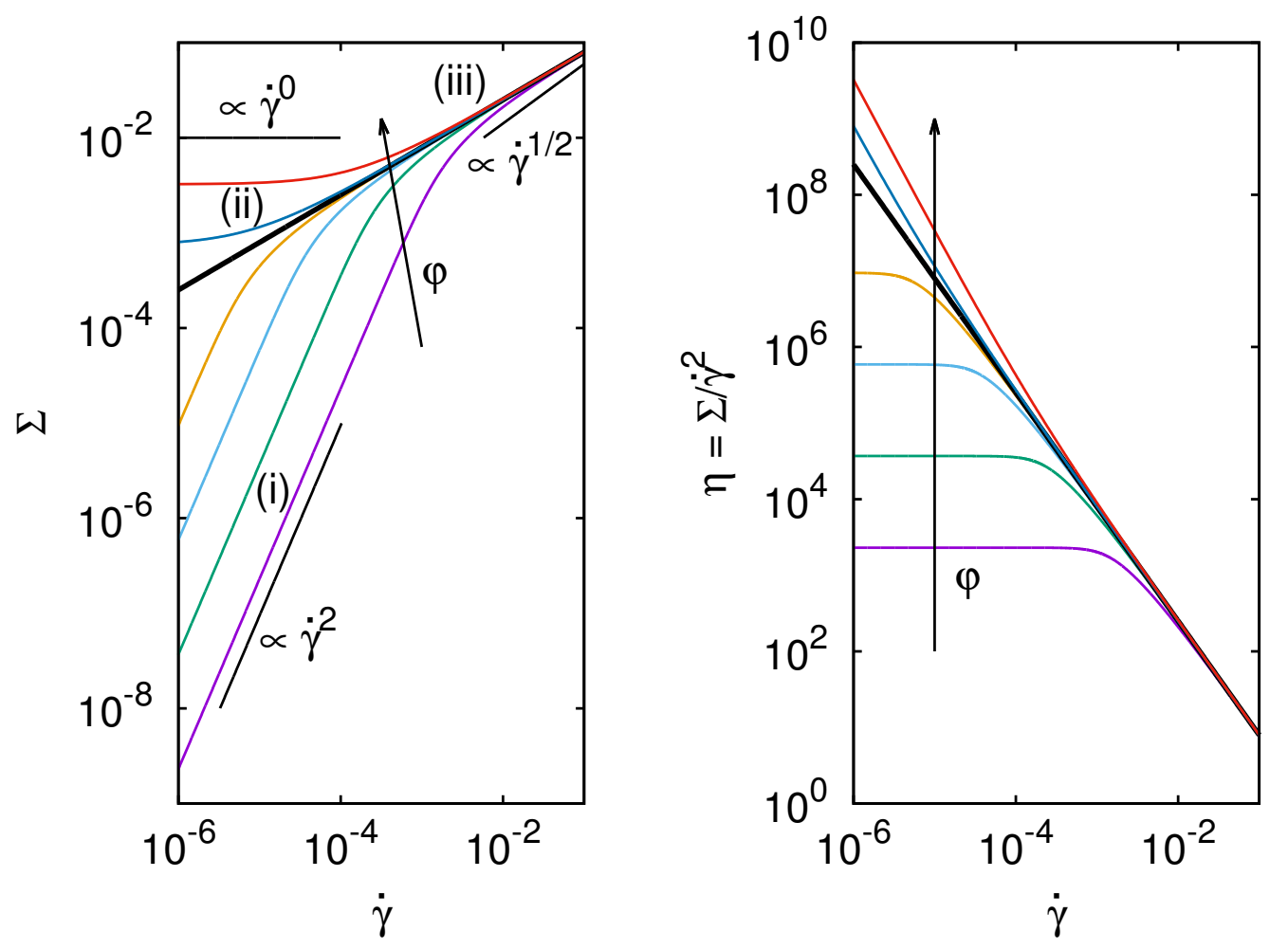

Figure 5.2.: Left: Schematic flow curves according to the model in Grob et al. [2014, mimicking frictionless particle flow. The arrow indicates increasing packing fraction and $\phi_{\mathrm{J}}$ is marked by a thick line. Three flow regimes are measured: (i) inertial flow, (ii) quasistatic flow, and (iii) elastic-inertial flow. Right: Generalized viscosity corresponding to the flow curves on the left. Units set by the particle properties according to Grob et al. 2014. 


\section{Shear rheology}

the inertial regime, the generalized viscosity plateaus at a value that depends on the packing fraction. As $\phi_{\mathrm{J}}$ is approached, the height of the plateau diverges in the limit of zero strain rate. Both, viscosity and yield stress, allow for estimates of the jamming packing fraction $\phi_{\mathrm{J}}$.

The interplay of time scales set by the experimental setup, i.e., strain rate or shear stress, and particle stiffness determine the rheology. The elastic properties of the constituents tune the flow characteristics and crossovers between the regimes [Campbell, 2002].

\subsection{Inertial flow}

Stiff grains or slow deformation imply that the time scale set by the stiffness of the particles, $\sqrt{k^{-1}}$, is smaller than the time scale set by the strain rate, $\dot{\gamma}^{-1}$. When the only relevant time scale is set by the strain rate, i.e., the deformation can be considered as sufficiently slow or the grains as sufficiently hard, stress scales as Bagnold, 1954

$$
\Sigma \propto \dot{\gamma}^{2}
$$

Inertial flow can be thought of as ballistic motion of grains interrupted by collisions. It is this ballistic motion that governs the momentum transport in the inertial flow regime Bagnold, 1954; Campbell, 2011]. Numerical simulations of soft spheres show that, in the inertial flow regime, the duration of collisions approaches the expected binary collision time set by the model parameters of the particles Campbell, 2002. Therefore, collisions are mainly binary. The binary character of collisions is a necessary precondition for the applicability of the kinetic theory of granular gases as a description of flow, see Brilliantov and Pöschel [2010]. In the kinetic theory, particles are modeled by infinitely stiff spheres, i.e., hard spheres, which collide with a collision rule and a coefficient of restitution. Hard spheres do not overlap and collisions are instantaneous. In practice, the particle stiffness is finite and therefore the hard sphere limit cannot be reached.

A measure to characterize the ratio of inertial forces - set by the strain rate to confining forces - set by the pressure in the system, $P$ - is the dimensionless 
inertial number Midi, 2004; Da Cruz, 2004; Da Cruz et al., 2005:

$$
I=\dot{\gamma} \sqrt{m / P}
$$

with particles of mass $m$. In contrast to the kinetic theory of granular gases, this rheological approach is a route of modeling flow that incorporates the finite particle stiffness and does not rely on a collision mechanism. In a hard particle system, inertia dominates since interparticle forces are absent in instantaneous collisions, i.e., the inertial number is large. In a system of soft particles, this is not necessarily true and confining forces can dominate. Therefore, the inertial number is used to describe several flow regimes, not only inertial flow. The inertial number proved to be a useful tool for the description of constitutive equations and, in particular, of friction laws that relate macroscopic shear stress to pressure, e.g., $\mu=\Sigma / P$ Midi, 2004, Da Cruz et al. 2005, Chialvo et al., 2012. The friction laws are known as $\mu(I)$-rheology and used in hydrodynamic descriptions of granular flow, see, e.g., Jop et al. 2006. However, for fluid mechanics applications, the simple $\mu(I)$-rheology is of limited applicability since it is mathematically ill-posed for large and low inertial numbers Barker et al. 2015. Non-local rheology, which can be regarded as series expansions where heterogeneities are taken into account, extends the idea of $\mu(I)$-rheology Volfson et al., 2003, Aranson et al., 2008, Bouzid et al., 2013, 2015].

\subsection{Plastic flow}

Plastic flow is subdivided into quasistatic flow and elastic-inertial flow. In the quasistatic flow regime, the strain rate is low and only the time scale set by the stiffness of the particles is relevant. The particle stiffness and packing fraction determine the stress plateau in the flow curve. The value of the stress plateau equals the yield stress:

$$
\Sigma=\Sigma_{\mathrm{Y}}
$$

In the elastic-inertial flow, the shear stress depends on the strain rate as a square root, see, e.g., Bocquet et al., 2009, Lemaître and Caroli, 2009, Olsson and Teitel, 


\section{Shear rheology}

2012; Chialvo et al. 2012, as shown in figure 5.2 (left, iii):

$$
\Sigma \propto \dot{\gamma}^{1 / 2}
$$

In the plastic flow regime, the particles' elastic properties are relevant and interparticle contacts are transmitters of stress and momentum Campbell, 2006. The contact duration exceeds the binary collision time by up to two orders of magnitude and contacts are not binary [Campbell, 2002]. This requires flow models other than the kinetic theory of granular gases and $\mu(I)$-rheology.

Early work on the plastic deformation of metallic glasses led to the picture that irreversible plastic events release stress in localized regions of size of a few particles - in so-called shear transformation zones Argon, 1979. The irreversible yielding of a shear transformation zone is called shear transformation. The idea of shear transformations was developed further to describe plastic flow and macroscopic yielding. Plastic flow is considered as a succession of elastic deformations, which accumulate potential energy, until shear transformations release energy. Shear transformations trigger each other in a cascade Maloney and Lemaître, 2006]. When average characteristics of shear transformations are incorporated in a description of flow, even macroscopic yielding of a jammed medium upon stress increase can be explained [Falk and Langer, 1997]. A similar description is used to describe the dynamics of locally yielding zones in generic soft glassy matter [Sollich et al. 1997]. The authors point out that many soft materials show structural disorder and metastability, which lead to the glass like behavior when deformed. A characteristic length for cooperativity larger than the size of the molecules was evidenced in experiments [Goyon et al., 2008]. In line with this insight, a nonlocal constitutive law with a local rate of plastic events has been derived Bocquet et al. 2009]. The non-locality is expressed by a flow cooperativity length that diverges in the quasistatic limit of zero strain rate. This implies finite size effects of flow and a dynamic yield stress at a critical point for a second-order phase transition. Elastic deformations interrupted by plastic rearrangements and a growing correlation length have been reported in several studies of dense flows, e.g., Heussinger and Barrat, 2009]. 


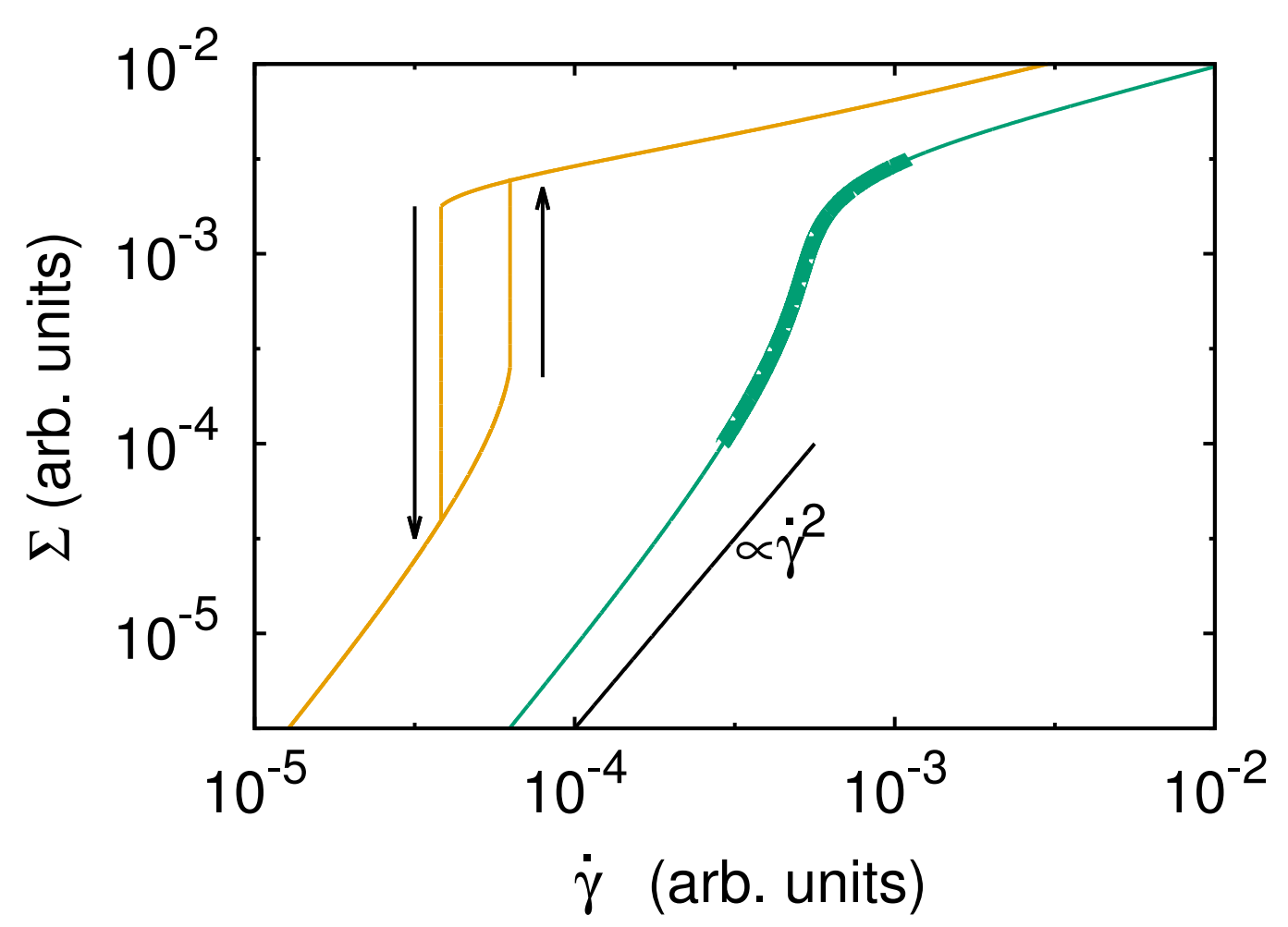

Figure 5.3.: Flow curves of a shear thickening fluid display continuous shear thickening (green) and discontinuous shear thickening (brown). The arrows indicate possible hysteresis in the discontinuous scenario.

\subsection{Shear thickening}

Flow regimes, e.g., inertial and plastic flow, differ fundamentally from each other with respect to transport of particles, momentum, and stress. The transitions between flow regimes are highly debated since the triggering mechanisms can be manifold. Shear thickening is such a transition and describes the increase of viscosity with shear stress or strain rate. Figure 5.3 shows potential flow curves of a shear thickening fluid. The scenario in figure 5.3 (green thick line) with a flow curve with finite, positive, and excessive increase (in this logarithmic representation a slope larger than 2), is termed continuous shear thickening. When the flow curve exhibits parts with finite jumps shear thickening is discontinuous, see figure 5.3 (brown line). In the discontinuous scenario, the continuous increase or decrease of the strain rate leads to jumps from one flow branch to the other, as indicated by the arrows in figure 5.3 . The shear stress, and thereby the viscosity, changes discontinuously. The phenomenon of shear thickening is absent in the 


\section{Shear rheology}

flow curves of frictionless granular matter as illustrated in figure 5.2 .

Approaches that try to explain shear thickening of a complex fluid and the shape of a flow curve, as shown in figure 5.3, stem from a broad variety of studies. In the following, we discuss studies that exhibit shear thickening but differ by the (experimentally, numerically, or analytically) examined system. Experiments are mainly conducted on suspensions, i.e., heterogeneous mixtures of solid particles floating in a solvent. Examples are an aqueous solution with suspended photoelastic disks or micro meter sized silica spheres. These examples possess an important difference: large photoelastic disks are granular grains and athermal contrasting small silica spheres that are only of micro meter size and experience Brownian motion Brown, 1828. Solid Brownian particles, e.g., micro meter sized silica spheres or micelles suspended in a solvent, are called colloids.

A general and abstract argument for discontinuous shear thickening flow curves is the coexistence of differently flowing phases within the system. In this case, local flow curves for each phase add up to the macroscopic flow curve that can be described by a so-called s-shaped or sigmoidal flow curve Olmsted, 1999. In particular, shear localization leads to non-monotonic flow curves Olmsted, 2008, Schall and van Hecke, 2010]. Indeed, localized phases are observed in experiments and are accompanied with shear thickening. In micellar solution, shear thickening is associated with localization of an anisotropic phase as an evidence of coexisting phases Berret et al. 2002. In dense suspensions, shear thickening and shear jamming are reported, but both pinned to shear localization Fall et al., 2008. The authors argue that shear jamming results from dilatancy and confinement, i.e., shear thickening turns into shear jamming because the system's finite size.

In this paragraph, we treat arguments that rely on the presence of a suspension. An explanation for shear thickening suspensions are hydrodynamic interactions [Wagner and Brady, 2009]. Dynamically correlated clusters, which enhance the particles non-affine motion and thereby enhance dissipation, are another mechanism Andreotti et al., 2012, Heussinger, 2013. In suspensions, where the inertia of colloids is not negligible, shear thickening is argued to be due to anisotropy in the microstructure which creates an effectively larger packing fraction Picano et al., 2013. A conclusive phase diagram, including a shear thickening regime, for Newtonian suspensions with friction and inertia was proposed recently but does not give an explanation for flows of dry granular media [Ness and Sun, 2015. 
Also, the contact network between the particles is an important mediator for shear thickening. In this paragraph, we deal with the importance of contacts. The role of frictional contacts is highlighted by rheometry with PMMA spheres, numerical simulations Guy et al., 2015, Ness and Sun, 2016, and with colloidal silica particles Royer et al. 2016. Shear thickening is explained by different mechanisms of particle contacts Wyart and Cates, 2014]: When particles are pushed together with large enough forces, frictional contacts become relevant and support larger load than lubricated contacts of particles which are just slightly pushed together. However, it remains elusive which kind of shear thickening, i.e., continuous or discontinuous, is realized in an experiment or simulation and by which mechanism it is implied. Contact forces dominate continuous shear thickening in suspension, as the mildest ${ }^{3}$ form of shear thickening Lin et al., 2015. In Fernandez et al. 2013; ; Mari et al. 2014, both, continuous and discontinuous shear thickening, were identified to occur based on frictional contacts and lubrication forces but controlled by packing fraction, i.e., shear thickening becomes stronger when the packing fraction increases and eventually turns from continuous to discontinuous. The packing fraction controls the number of contacts and thus the packing fraction is a crucial control parameter for shear thickening media, see also Brown and Jaeger 2009; Seto et al. 2013. The discontinuous scenario is accompanied with phenomenology reminiscent of first-order phase transitions in equilibrium statistical mechanics, e.g., hysteresis in simulations of dry granular media Otsuki and Hayakawa, 2011. Moreover, the interaction mechanisms that is minimally required for shear thickening is controversial. In Wyart and Cates 2014, the authors argued that a microscopic stress scale, which distinguishes lubricated from frictional contacts, is necessary for shear thickening. In contrast, in Otsuki and Hayakawa 2011, the authors do not dwell on such a mechanisms but use the same simplified approach as presented in Chapter 3 .

As the discussion points out, shear thickening is observed in different experimental, numerical, and theoretical investigations. A host of different mechanisms are found to be the origin for shear thickening, e.g., friction, lubrication forces, correlated cluster, shear localization, and other heterogeneities. Heterogeneities lead to gradients and result in unsteady flow. Heterogeneities and unsteady flow have not been discussed in dry granular media. Also, many of the arguments

\footnotetext{
${ }^{3}$ The viscosity grows less in continuous shear thickening than in the discontinuous shear thickening.
} 


\section{Shear rheology}

above do not hold when the suspending fluid has zero or negligible viscosity.

\subsection{Unsteady flow}

The sections on inertial flow 5.2 and on plastic flow 5.3 deal with flow states that do not show time dependent behavior - except for fluctuations. Due to the fact that the flowing medium is composed of grains, discrete interactions, i.e., collisions, make fluctuations an inherent feature of the dynamics and thus will not be considered as a signature of unsteady flow. The flow is steady when the system is tuned in the inertial or plastic flow regime. In the proximity of transitions between inertial or plastic flow, unsteady behavior can emerge. Three possible scenarios for unsteady flow are presented in the following:

(i) the coexistence of states, which are realized alternately as time progresses,

(ii) oscillations with a systematic pattern, and

(iii) chaotic response and irregular dynamics.

A rigorous characterization of these different scenarios is far from trivial and thus a distinction between these states is a delicate task and not always unambiguous. Figure 5.4 shows time series of the different unsteady flow scenarios.

Coexistence is given when metastable states are realized alternately, e.g., in the course of an experiment or a simulation, two differently flowing phases alternate, see figure 5.4 (i). In numerical studies of frictional particles, metastable flow states are probed and successive alternations between plastic and inertial flow are observed near a critical packing fraction Aharonov and Sparks, 1999; Mari et al., 2014. Similar features are found in three dimensional simulations, where the evolution of contact number and pressure are shown to be linked; both fluctuate the most at a critical packing fraction Chialvo et al., 2012. Metastable states and hysteresis due to shear localization are found experimentally, e.g., Chen et al., 1992; Berret and Porte, 1999]. In a shear thickening regime, the stress distribution shows two peaks, which is interpreted as coexistence of differently flowing states Heussinger, 2013. Therefore, the shear thickening regime presented by the author is only apparent as it is constituted not by persistent, but alternating flow states. 


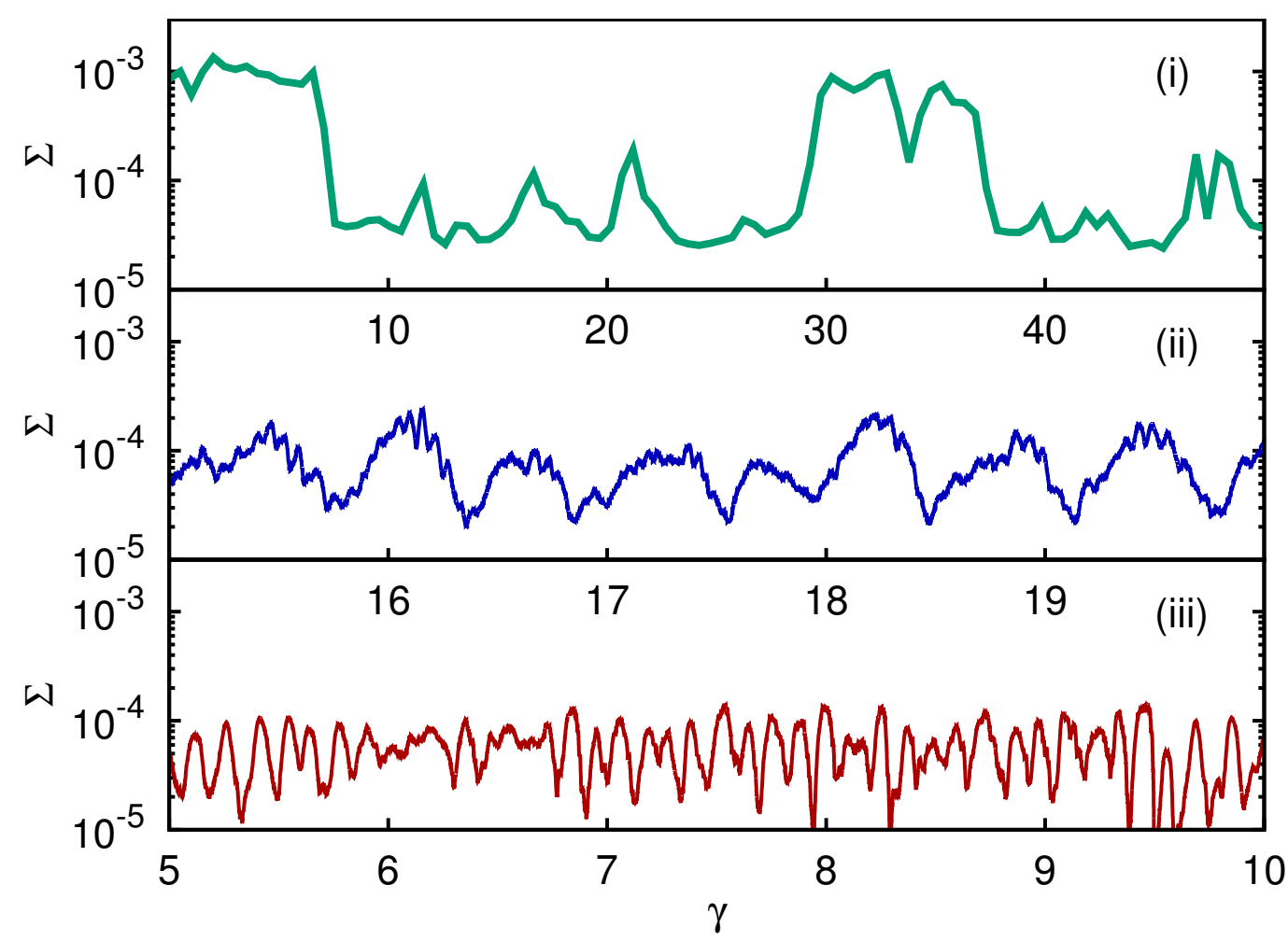

Figure 5.4.: Different scenarios for unsteady flow: (i) coexistence, (ii) flow with systematic patterns, and (iii) chaotic response. Units set by the particle properties according to Grob et al. 2014]. Simulation parameters: $\phi=0.7975$ and (i) $N=8000$, imposed strain rate $\dot{\gamma}=1.1 \times 10^{-4}$, (ii) $N=80000$, imposed strain rate $\dot{\gamma}=1.25 \times 10^{-4}$, and (iii) $N=80000$, imposed shear stress $\Sigma=6.28 \times 10^{-5}$. 


\section{Shear rheology}

Oscillations with a systematic pattern are another realization of unsteady flow, see figure 5.4 (ii). A discussion on sigmoidal flow curves, their relation to frictional contacts and the potential of shear instabilities leading to oscillations is given in Bashkirtseva et al. 2009. The authors also present a phenomenological model for the flow of a complex fluid, which, locally, shows a nonlinear viscoelastic response to stress - ultimately leading to global oscillations. Colloidal experiments show non-Gaussian stress fluctuations with heavy tailed distributions when the system is driven in an apparent shear thickening regime [Lootens et al. 2003]. Experiments on micellar solutions evidence shear thickening, hysteresis and oscillating behavior Fischer, 2000; Fernández et al., 2009; Lutz-Bueno et al., 2013. The authors conclude that the system does not settle into a steady state because different phases with different flow characteristics exist. The emergence and destruction of these phases are dynamically induced by shear in an unstable flow regime. Time dependent flow is found near jamming of gravity driven flow down an incline and shows temporally heterogeneous dynamics and intermittent behavior characterized by spatial heterogeneities visible in the contact network [Silbert, 2005]. A simple rheological model predicts oscillatory flow and a sigmoidal flow curve Head et al., 2001, 2002].

The scenario of flow with chaotic dynamics, see figure 5.4 (iii), appears to be the least documented. A simple scalar model of a shear thickening material predicts rheological chaos as a result of flow instabilities Cates et al. 2002. Also, fluid dynamic models show complex rheological behavior in shear thickening states Hess et al. 2006. In corn starch experiments, unpredictable rheological response is measured where discontinuous shear thickening is expected Hermes et al. 2015. 


\section{Active microrheology}

Microrheology examines rheological properties in complex fluids by the dynamics of a suspended probe particle. A comprehensive review is given by Squires and Mason 2010]. In so-called passive microrheology, the motion of the probe particle is induced by the temperature of the suspending fluid, $T$. The simplest microrheological equation is the Stokes-Einstein relation, equation 6.1, which relates the viscosity of a Newtonian fluid in equilibrium, $\eta$, to the diffusion constant, $D$ :

$$
D=\frac{k_{\mathrm{B}} T}{6 \pi r \eta}
$$

with the Boltzmann constant, $k_{\mathrm{B}}$, and the radius of the probe particle, $r$ Einstein, 1905; Sutherland, 1905]. The mean square displacement expressed in terms of the diffusion constant, $\left\langle\Delta x^{2}(t)\right\rangle=2 D t$, is the link between the position of the probe particle as a microscopic quantity and the viscosity as a rheological quantity. Thereby, the Newtonian viscosity can be inferred from the probe particle's fluctuations. Seminal work by Mason and Weitz 1995] examines the relation of the mean square displacement and the frequency dependent complex shear modulus, $G^{\star}(\omega)$ ] of a non-Newtonian fluid, which describes the linear rheological relation between shear stress and shear strain, completely [Mason and Weitz, 1995].

When external driving is applied to the probe particle microrheology is called active. The active forcing allows to go beyond the linear-response regime and to examine linear and nonlinear rheological response. Nonlinear phenomena are, e.g., shear thinning, shear thickening, and the granular glass transition, a rigidity transition of a stochastically driven granular fluid Kranz et al., 2010, Sperl et al. 2012; Kranz et al., 2013]. To study the granular rigidity transition, Candelier and Dauchot 2009, 2010] conducted experiments on a horizontal layer of vibrated granular particles and examined the dynamics of an externally driven

\footnotetext{
${ }^{1}$ Real and imaginary part of $G^{\star}(\omega)$ correspond to the elastic and viscous modulus, respectively.
} 


\section{Active microrheology}

probe particle. Two qualitatively different regimes, seperated by a packing fraction dependent force threshold, which diverges at the granular glass transition, are reported: a fluidized regime with continuous motion and a solid-like regime with intermittend motion.

In general, the external driving is realized with a force, $F$, or a velocity, $v$, which is imposed on the probe particle and the complementary quantity is measured. This gives access to the mobility, $\nu$, via the velocity-force relation

$$
v=\nu F
$$

A velocity-force relation is in general nonlinear due to force thickening, i.e., a sublinear velocity-force relation, or force thinning, i.e., a superlinear velocity-force relation. In this context, the inverse of the mobility is called friction coefficient, ל2. The frequency dependent generalized Stokes mobility, $\nu^{\star}(\omega)$, relates the mobility to the complex viscosity, $\eta^{\star}(\omega)=-i \omega^{-1} G^{\star}(\omega)$ :

$$
\nu^{\star}(\omega)=\frac{\eta}{\eta^{\star}(\omega)} \nu
$$

with the frequency, $\omega$, which is set by the velocity of the probe particle.

In shear rheology, as discussed in Chapter 5 , the strain rate is homogeneous (in a stationary state in simple shear geometry). In microrheology, however, shear is applied locally, i.e., the medium near the probe particle can be far out of equilibrium while further away from the probe particle, the medium can still remain undeformed or unstressed. Therefore, a quantitative comparison between shear rheology and microrheology is, in general, not appropriate. Nevertheless, microrheology gives insight into microstructural properties in force thinning regimes [Sriram et al., 2009] or growing length scales close to jamming (or the granular glass transition) Candelier and Dauchot, 2009, 2010]. The review Reichhardt and Reichhardt 2014 discusses what can be learned about jamming with the help of externally driven probes and Puertas and Voigtmann 2014 review microrheology on colloids and discuss granular systems, too.

\footnotetext{
${ }^{2}$ The friction coefficient is not to be confused with Coulomb's friction parameter, which is called $\mu$.
} 


\section{Part II.}

\section{Results}



This part contains the results of this thesis, which are presented as separate articles.

Chapter 7 contains the main achievement of this thesis: studies on sheared frictional granular media. In section 7.1, we present the article "Jamming of frictional particles: A nonequilibium first-order phase transition", Grob et al., 2014], which discusses the jamming transition of sheared frictional granular particles in small simulation cells. The article in section 7.2 , "Rheological chaos of frictional grains", Grob et al., 2016], treats unsteady flow near a shear induced jamming transition in large simulation cells. We present numerical findings that are in accordance with the prediction of a simple model that is developed in the same study. The stability analysis of the solutions of the model is presented in appendix A. In section 7.3, we present the manuscript "Unsteady rheology and heterogeneous flow of dry frictional grains", a detailed study, which links the preceding studies together and provides a detailed description of heterogeneities and time dependent flow close to jamming.

Chapter 8 contains results of a study that was carried out as a side project during the period of this thesis. The numerical foundations of this study, which was developed before the period of this thesis, is described in [Fiege et al. 2012]. We use an event-driven simulation scheme that accounts for a resistive drag force ${ }^{3}$ and introduces an actively driven probe particle of which we investigate the nonlinear velocity-force relation close to the granular glass transition. The numerical results are related to analytical results by Wang and Sperl in the article "Active microrheology of driven granular particles" Wang et al. 2014.

\footnotetext{
${ }^{3}$ When a particle moves ballistically with velocity, $v$, a drag force, $F_{\mathrm{D}}$, is a resistive force proportional to the particle's velocity: $F_{\mathrm{D}} \propto-v$; it is motivated by, e.g., a fluid that surrounds the particles or, in a dry system, friction with a bottom plate on which the experiment is built.
} 



\section{Shear rheology of frictional grains}

\subsection{Jamming of frictional particles: $A$ nonequilibium first-order phase transition}

Reprinted article with permission from

Grob, Matthias and Heussinger, Claus and Zippelius, Annette

Physical Review E 89050201 (2014)

http://dx.doi.org/10.1103/PhysRevE.89.050201

Copyright (2014) by the American Physical Society. 



\title{
Jamming of frictional particles: A nonequilibrium first-order phase transition
}

\author{
Matthias Grob, ${ }^{1}$ Claus Heussinger, ${ }^{2}$ and Annette Zippelius ${ }^{1,2}$ \\ ${ }^{1}$ Max Planck Institute for Dynamics and Self-Organization, Am Faßberg 17, 37073 Göttingen, Germany \\ ${ }^{2}$ Institute for Theoretical Physics, Georg-August University of Göttingen, Friedrich-Hund Platz 1, 37077 Göttingen, Germany
}

(Received 21 November 2013; revised manuscript received 12 February 2014; published 27 May 2014)

\begin{abstract}
We propose a phase diagram for the shear flow of dry granular particles in two dimensions based on simulations and a phenomenological Landau theory for a nonequilibrium first-order phase transition. Our approach incorporates both frictional as well as frictionless particles. The most important feature of the frictional phase diagram is reentrant flow and a critical jamming point at finite stress. In the frictionless limit the regime of reentrance vanishes and the jamming transition is continuous with a critical point at zero stress. The jamming phase diagrams derived from the model agree with the experiments of Bi et al. [Nature (London) 480, 355 (2011)] and brings together previously conflicting numerical results.
\end{abstract}

DOI: 10.1103/PhysRevE.89.050201

PACS number(s): 45.70.-n, 66.20.Cy, 83.60.Rs

Random close packing is the point at which hard spherical—and frictionless_-particles generally jam into a stable heap. It is now known that the precise close-packing density $\varphi_{\text {rcp }}$ depends on the preparation protocol [1]. Nevertheless, this variability is small when compared to frictional systems, i.e., systems where particles not only transmit normal forces but also tangential forces among themselves. Indeed, frictional systems can jam at densities anywhere between random-close, random-loose, or even random-very-loose packing [2,3]. In this Rapid Communication we deal with the flow properties of frictional granular systems, where the jamming transition can be studied by monitoring the flow curves, i.e., the stressstrain rate relations $\sigma(\dot{\gamma})$. Previous simulations performed in the hard-particle limit $[4,5]$ do not observe any qualitative difference between frictionless and frictional systems, other than a mere shift of the critical density from $\varphi_{\text {rcp }}$ to $\varphi_{J}(\mu)$, which depends on the friction coefficient $\mu$ of the particles. Similar results, accounting for particle stiffness, are presented in Refs. [6,7]. Quite in contrast, Otsuki et al. [8] recently observed a discontinuous jump in the flow curves of the frictional system, which is absent in the frictionless analog [9]. In addition, they find not one but three characteristic densities for the jamming transition, which degenerate into random close packing when $\mu \rightarrow 0$. Similarly, Ciamarra et al. [10] observe three (but different) jamming transitions. Experimentally, Bi et al. [11] present a jamming phase diagram with a nontrivial (reentrance) topology that is not present in the frictionless scenario.

These latter results hint at friction being a nontrivial and indeed "relevant" perturbation to the jamming behavior of granular particles. Unfortunately, several inconsistencies remain unresolved. For example, the phase diagram in [10] is different from [11] and does not show stress jumps as observed in [8]. This points towards a more fundamental lack of understanding of the specific role of friction in these systems. What is the difference between frictional and frictionless jamming? By combining mathematical modeling with strain- and stress-controlled simulations we propose a jamming scenario that not only encompasses frictional as well as frictionless systems, but also allows one to bring together previously conflicting results.

We simulate a two-dimensional system of $N=8000$ soft, frictional particles in a square box of linear dimension $L$. The particles all have the same mass $m=1$, but are polydisperse in size: 2000 particles each for diameter $d=0.7,0.8,0.9,1.0$. The particle volume fraction is defined as $\varphi=\sum_{i=1}^{N} \pi d_{i}^{2} / 4 L^{2}$. Normal and tangential forces, $\boldsymbol{f}^{(n)}$ and $\boldsymbol{f}^{(t)}$, are modeled with linear springs of unit strength for both elastic as well as viscous contributions. (Thereby units of time, length, and mass have been fixed.) Coulomb friction is implemented with friction parameter $\mu=2$ [12]. In the strain-controlled simulations, we prepare the system with a velocity profile $\boldsymbol{v}_{\text {flow }}=\dot{\gamma}(0) y \hat{\boldsymbol{e}}_{x}$ initially. Subsequently the shear rate is implemented with Lees-Edwards boundary conditions [13] until a total strain of $200 \%$ is achieved after time $T$. Whenever the strain rate is changed to a new value, we wait for a time $\sim 0.5 T$ to allow for the decay of transients. In the stress-controlled simulations, a boundary layer of particles is frozen and the boundary at the top is moved with a force $\sigma L \hat{\boldsymbol{e}}_{x}$, whereas the bottom plate remains at rest.

In the strain-controlled simulations we impose the strain rate $\dot{\gamma}$ and measure the response, the shear stress $\sigma(\dot{\gamma})$, for a range of packing fractions $0.78 \leqslant \varphi \leqslant 0.82$. Thereby the system is forced to flow for all packing fractions; the resulting flow curves are shown in Fig. 1.

We observe three different regimes. For low packing fraction, the system shows a smooth crossover from Bagnold scaling, $\sigma=\eta \dot{\gamma}^{2}$ (called "inertial flow") to $\sigma \propto \dot{\gamma}^{1 / 2}$ (called "plastic flow"). As the packing fraction is increased, we observe a transition to hysteretic behavior [8]: Decreasing the strain rate from high values, the system jumps discontinuously to the lower branch. Similarly, increasing the strain rate from low values, a jump to the upper branch is observed. A well developed hysteresis loop is shown in the inset of Fig. 1. The onset of hysteresis defines the critical density $\varphi_{c}$. We estimate its value $\varphi_{c}$ between 0.7925 and 0.795 by visual inspection of the flow curves as described in the Supplemental Material [14]. As $\varphi$ is increased beyond the critical value $\varphi_{c}$, the jump to the lower branch happens at smaller and smaller $\dot{\gamma}$, until at $\varphi_{\sigma}$, the upper branch first extends to zero strain rate, implying the existence of a yield stress, $\sigma_{\text {yield }}$. For $\varphi_{c}<\varphi<\varphi_{\sigma}$, the strain rate for the jump to the lower branch, $\dot{\gamma}_{\sigma} \propto \varphi_{\sigma}-\varphi$, scales linearly with the distance to $\varphi_{\sigma}$ which allows us to determine $\varphi_{\sigma} \cong 0.8003$. Finally at $\varphi_{\eta}$, the generalized viscosity $\eta=\sigma / \dot{\gamma}^{2}$ diverges and for $\varphi>\varphi_{\eta}$ only plastic flow is observed. The scaling of the viscosity $\eta \propto\left(\varphi_{\eta}-\varphi\right)^{-4}$ is 


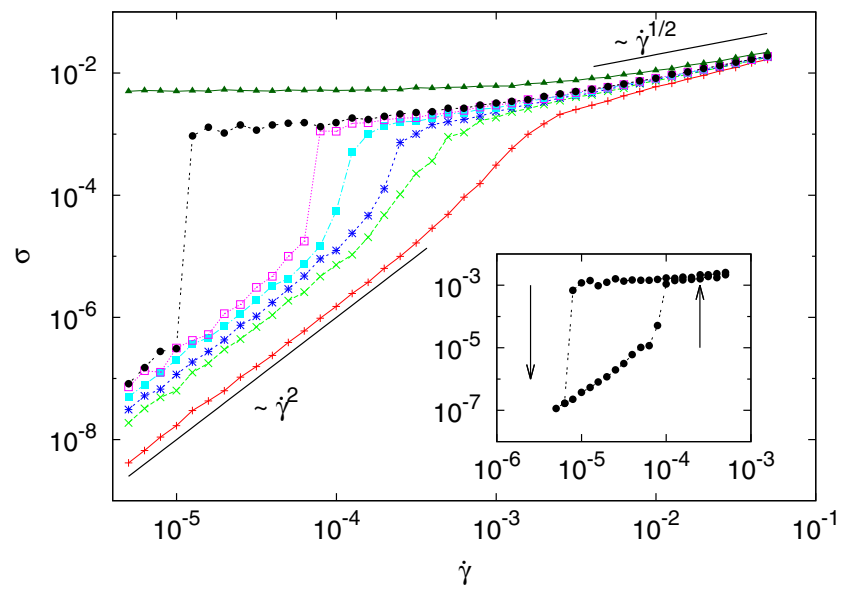

FIG. 1. (Color online) Flow curves $\sigma(\dot{\gamma})$ for different packing fractions $\varphi=0.78,0.7925,0.795,0.7975,0.79875,0.80,0.82$ (from bottom to top). Main part: Flow curves obtained by decreasing $\dot{\gamma}$. Inset: Example of a hysteresis loop for $\varphi=0.80$.

in agreement with previous results [8] and yields $\phi_{\eta} \cong 0.819$. Note that all three packing fractions are well separated, and furthermore, $\varphi_{\eta}$ is still well below the frictionless jamming density at random close packing $\varphi_{\text {rcp }} \cong 0.8433$. The scaling plots $\dot{\gamma}_{\sigma}$ and $\eta$ are shown in the Supplemental Material [14].

All the observations can be explained in the framework of a simple model, which can be viewed as a phenomenological Landau theory, that interpolates smoothly between the inertial and the plastic flow regime:

$$
\dot{\gamma}(\sigma)=a \sigma^{1 / 2}-b \sigma+c \sigma^{2}
$$

where $a, b, c$ are coefficients which in general depend on the packing fraction. Equation (1) can be taken to result from a class of constitutive models that combine hydrodynamic conservation laws with a microstructural evolution equation [15], or from mode-coupling approaches [16].

The numerical data suggest that the plastic flow regime is only weakly density dependent for packing fractions considered here, so we take $c$ to be independent of $\varphi$ for simplicity. In the inertial flow regime, on the other hand, we expect to see a divergence of the shear viscosity at $\varphi_{\eta}$, implying that the coefficient $a$ of our model vanishes at $\varphi_{\eta}$ and changes sign, $a=a(\varphi)=a_{0}\left|\varphi_{\eta}-\varphi\right|\left(\varphi_{\eta}-\varphi\right)$. The coefficient $b$ is assumed to be at most weakly density dependent.

The simple model predicts a discontinuous phase transition with a critical point in analogy to the van der Waals theory of the liquid-gas transition [see Fig. 2(left)]. The critical point is determined by locating a vertical inflection point in the flow curve. In other words we require $\partial_{\sigma} \dot{\gamma}=0$ and simultaneously $\partial_{\sigma \sigma} \dot{\gamma}=0$. These two equations together with the constitutive equation (1) determine the critical point: $b_{c}=\frac{3}{2} a\left(\varphi_{c}\right)^{2 / 3} c^{1 / 3}$ with the critical strain rate given by $\dot{\gamma}_{c}=\frac{3}{16} \frac{a^{4 / 3}}{c^{1 / 3}}$ and the critical stress $\sigma_{c}=\frac{1}{4}\left(\frac{a}{c}\right)^{2 / 3}$. For $\varphi>\varphi_{c}$, the model predicts an unstable region, where $\partial_{\sigma} \dot{\gamma}<0$. This is where the stress jump occurs in the simulations. The flow curves of the model are presented in Fig. 2(left), assuming $b \equiv b_{c}$, and fitting the two constants $c, a_{0}$ to the data. The model predicts a yield stress to first occur, when two (positive) zeros for the function $\dot{\gamma}(\sigma)=0$ coincide.
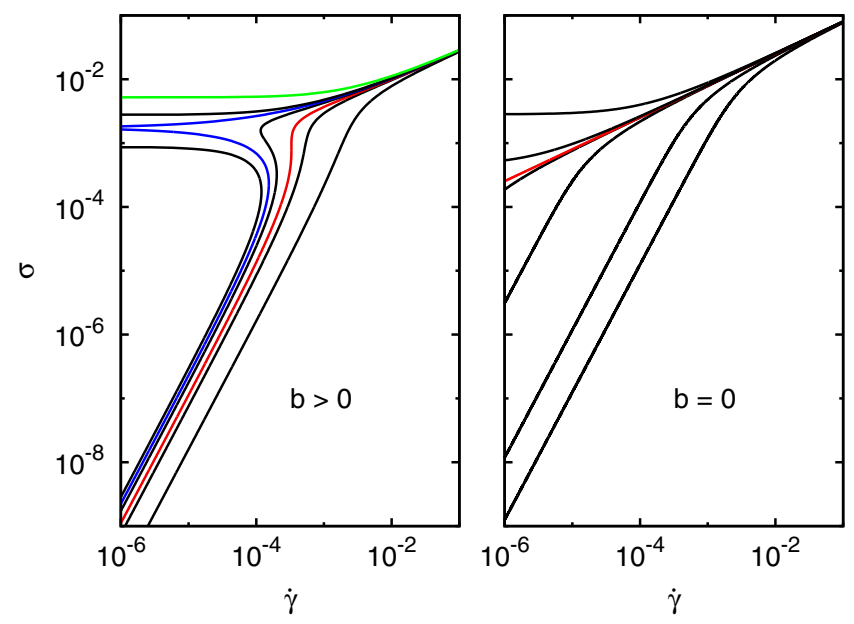

FIG. 2. (Color online) Flow curves of the simple model, Eq. (1). Left: Frictional scenario with range of packing fractions as in Fig. 1; $\phi_{c}$ is indicated by the red line, $\phi_{\sigma}$ by the blue line, and $\phi_{\eta}$ by the green line. Right: Flow curves for frictionless particles of the simple model implemented with $b=0$ and $\varphi_{c}=\varphi_{\sigma}=\varphi_{\eta}=0.8433$; critical flow curve in red.

This happens at a density $\varphi_{\sigma}$ determined implicitly by $a\left(\varphi_{c}\right)=$ $a\left(\varphi_{\sigma}\right) \sqrt{2}$ and the yield stress is given by $\sigma_{\text {yield }}=[a /(2 c)]^{2 / 3}$. The flow curves can be fitted better, if we allow for weakly density dependent coefficients $b$ and $c$. However, we refrain from such a fit, because even in its simplest form the model can account for all observed features qualitatively: a critical point at $\varphi_{c}$, the appearance of a yield stress at $\varphi_{\sigma}$, and the divergence of the viscosity at $\varphi_{\eta}$, ordered such that $\varphi_{c}<\varphi_{\sigma}<\varphi_{\eta}$. The flow curves for these three packing fractions are highlighted in Fig. 2 and further illustrated in the Supplemental Material [14].

The limiting case of frictionless particles can be reached by letting $\mu \rightarrow 0$. Simulations indicate that in this limit hysteretic effects vanish [8,9] and the jamming density is increased approaching random close packing. Within the model this transition can be understood in terms of the variation of two parameters: First $b(\mu) \rightarrow 0$ in Eq. (1) implies that the three densities $\left(\varphi_{c}, \varphi_{\sigma}, \varphi_{\eta}\right)$ coincide and second $\varphi_{\eta}(\mu) \rightarrow \varphi_{\text {rcp }} \cong$ 0.8433 . While a $\mu$-dependent $\varphi_{\eta}$ simply shifts the phase diagram towards higher densities, the parameter $b$ accounts for the more important changes of the topology of the phase diagram. The flow curves in this limit are presented in Fig. 2(right). They present a continuous jamming scenario consistent with previous simulations in inertial [9] as well as overdamped systems $[17,18]$.

What happens in the unstable region? Naively one might expect "coexistence" of the inertial and the plastic flow regime, i.e., shear banding. However, this would have to happen along the vorticity direction $[15,19]$, which is absent in our two-dimensional setting. Alternative possibilities range from oscillating to chaotic solutions [20,21]. We will see that, instead, the system stops flowing and jams at intermediate stress levels. Interestingly, this implies reentrance in the $(\sigma, \varphi)$ plane with a flowing state both for large and small stress, and a jammed state in between. 

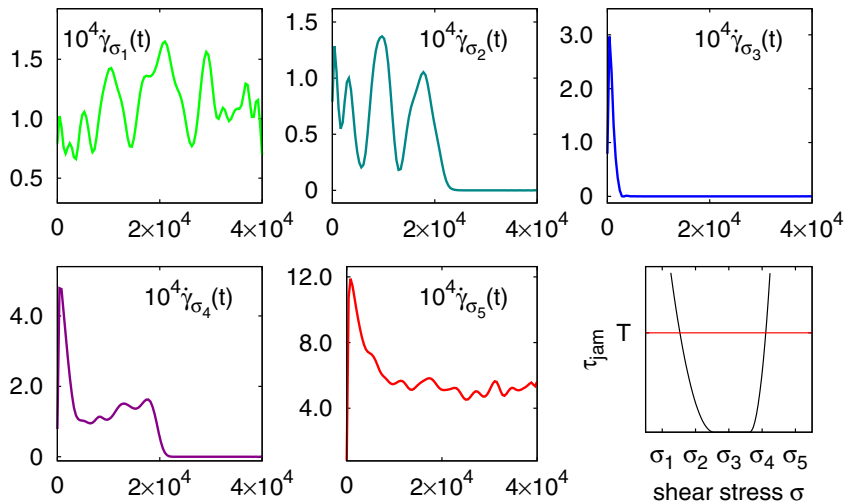

FIG. 3. (Color online) Time series of the strain rate with packing fraction $\varphi=0.7975$ and different, but fixed, stresses $\sigma_{1}<\sigma_{2}<\sigma_{3}<$ $\sigma_{4}<\sigma_{5}$ (stress values are indicated in Fig. 6). Lower right corner: Schematic picture of the jamming time being cut off at the simulation time $T$.

To address the unstable regime in more detail, we have performed stress-controlled simulations: The shear stress is imposed and we measure the strain rate as a function of time. The initial configurations are chosen with a flow profile corresponding to the largest strain rate in the inertial flow regime, which was observed previously in the strain-controlled simulations. For a fixed $\varphi$, several time series are shown in Fig. 3, representing the different regimes. The lowest value of $\sigma_{1}$ is chosen in the inertial flow regime, so that the system continues to flow for large times. Similarly $\sigma_{5}$ is chosen in the plastic flow regime and the system continues to flow as well. The intermediate value $\sigma_{3}$ is chosen in the unstable region and the system immediately jams. Between the jamming and the flow regime we find intermediate phases with transient flow that ultimately stops [10].

To quantify the different flow regimes, we introduce the time $\tau_{\text {jam }}$ the system needs to jam. Schematically we expect the result shown in the lower right corner of Fig. 3: In the jammed phase $\tau_{\text {jam }}=0$, whereas in the flow phase $\tau_{\text {jam }}$ is infinite. In between $\tau_{\text {jam }}$ is finite implying transient flow before the system jams. We expect $\tau_{\text {jam }}$ to go to zero as the jammed phase is approached and to diverge as the flow phases are approached. Given that the simulation is run for a finite time, the divergence should be cut off at the time of the simulation run, $T$, indicated by the horizontal (red) line in the schematic in the lower right corner of Fig. 3.

These expectations are borne out by the simulations: In Fig. 4 we show a contour plot of $\tau_{\text {jam }}$ as a function of $\varphi$ and $\sigma$. In the dark blue region, $\tau_{\text {jam }}$ is very small, corresponding to the jammed state. In the bright yellow region $\tau_{\text {jam }}$ exceeds the simulation time; hence this region is identified with the flow regime-inertial flow for small $\sigma$ and plastic flow for large $\sigma$. The intermediate (red) part of the figure corresponds to the transient flow regimes.

In our simple model, Eq. (1), the jammed state has to be identified with the unstable region. It seems furthermore suggestive to identify the transient flow regime with metastable regions. The phase diagram, as predicted by the simple model (with finite $b$ ) is shown in Fig. 5 (schematic). In the region

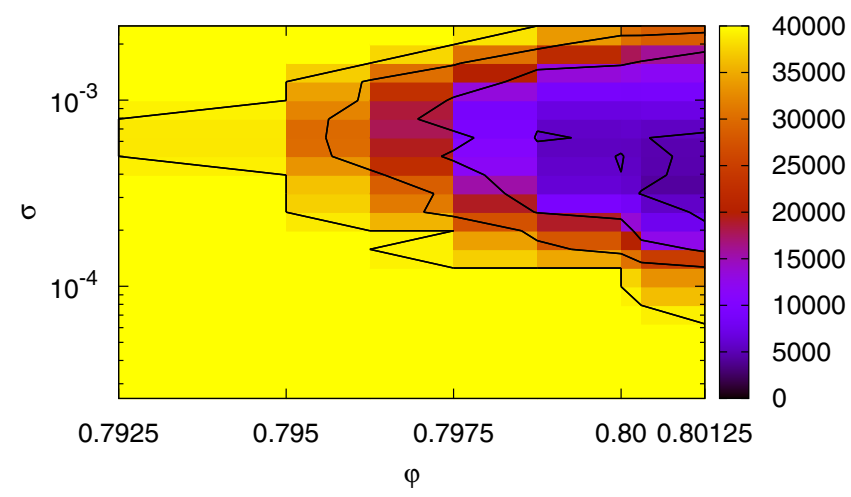

FIG. 4. (Color online) Numerical results for the phase diagram. The mean flow time $\tau_{\mathrm{jam}}$ is encoded with color. The flow phase is indicated in yellow (bright) and the jamming phase in blue (dark). Lines are contours of constant $\tau_{\mathrm{jam}}$.

within the (thick) red curve, Eq. (1) has no solution: the system jams. Outside the (thin) blue curve, the solution is unique corresponding to either inertial flow (low stress) or plastic flow (high stress). In between, in the shaded region, the equations allow for two solutions and hence metastable states. Jamming from these metastable states is discontinuous, i.e., the strain rate jumps to zero from a finite value. At a packing fraction $\varphi_{\sigma}$, a yield stress first appears and grows as $\varphi$ is increased further, giving rise to a kink in the red curve and a continuous jamming scenario. Beyond $\varphi_{\eta}$ inertial flow is no longer possible [22]. In the frictionless limit $b \rightarrow 0$ all these different packing fractions merge with $\varphi_{\text {rcp }}$ giving the phase diagram the simple structure well known from previous work [23] and shown in the inset in Fig. 5.

The presence of long transients is fully consistent with the results of Ref. [10]. Due to a restricted stress range in those simulations, however, only the upper part of the phase diagram is captured and the reentrance behavior is missed. To get a better understanding of these transients (or possibly

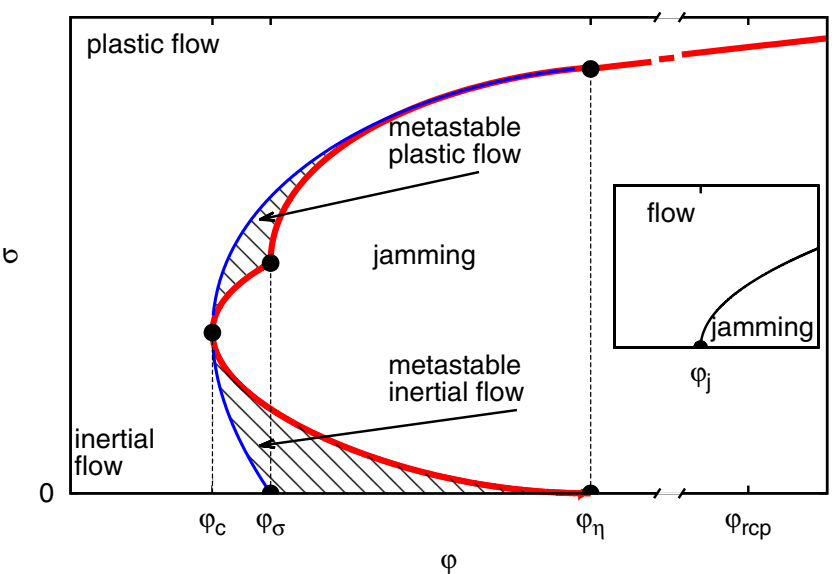

FIG. 5. (Color online) Phase diagram of the model (schematic), revealing reentrant flow for small and large $\sigma$, as well as flow and jam states in the "metastable" regions for frictional particles (main panel) and the known jamming phase diagram for frictionless particles (inset). 


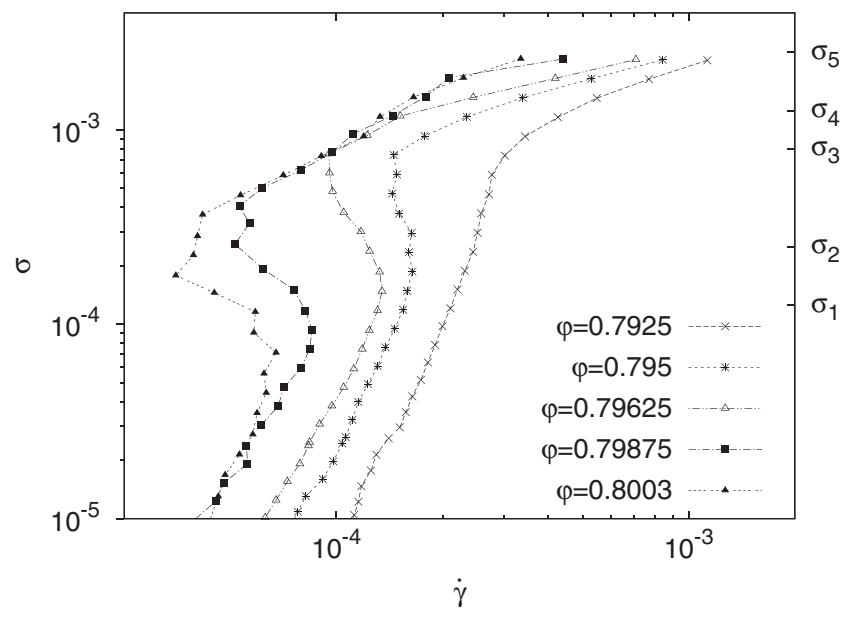

FIG. 6. Flow curves from the stress-controlled simulations. The unstable branches (decreasing stress) are obtained as time averages over the transient flow (right axis: stress values used for the time series in Fig. 3).

metastable states), we have tried to construct the flow curves in this regime by the following procedure: The monitored time series are truncated as soon as the system jams. The (transiently) flowing part of the time series is averaged over time, giving rise to the flow curves, shown in Fig. 6. These flow curves show clearly a nonunique relation $\sigma(\dot{\gamma})$ or equivalently a non monotonic relation $\dot{\gamma}(\sigma)$, which can only be observed as transient behavior, before the system has settled into a stationary state.

In conclusion, the goal of this Rapid Communication is to understand the role of friction in the jamming behavior of dry granular matter. To this end we present a theoretical model (supplemented by molecular dynamics simulations) that can reproduce all the phenomenology of simulated flow curves (Fig. 2) both for the fully frictional system as well as for the limiting case of frictionless particles. The jamming phase diagrams derived from the model agree with recent experiments [11]. The key result is that the transition between the two jamming scenarios, frictionless and continuous, and frictional and discontinuous, can in our model be accounted for by the variation of just a single parameter $(b)$. The most important feature of the frictional phase diagram is reentrant flow and a critical jamming point at finite stress. The fragile "shear jammed" states observed in the experiments [11] then correspond to the reentrant (inertial) flow regime in our theory. Our work allows one to bring together previously conflicting results $[6-8,10]$ and opens a new path towards a theoretical understanding of a unified jamming transition that encompasses both frictionless as well as frictional particles.

We thank Till Kranz for fruitful discussions. We gratefully acknowledge financial support by the DFG via FOR 1394 and the Emmy Noether program (He 6322/1-1).
[1] P. Chaudhuri, L. Berthier, and S. Sastry, Phys. Rev. Lett. 104, 165701 (2010).

[2] M. P. Ciamarra, P. Richard, M. Schröter, and B. P. Tighe, Soft Matter 8, 9731 (2012).

[3] C. Song, P. Wang, and H. A. Makse, Nature (London) 453, 629 (2008).

[4] F. da Cruz, S. Emam, M. Prochnow, J.-N. Roux, and F. Chevoir, Phys. Rev. E 72, 021309 (2005).

[5] M. Trulsson, B. Andreotti, and P. Claudin, Phys. Rev. Lett. 109, 118305 (2012).

[6] S. Chialvo, J. Sun, and S. Sundaresan, Phys. Rev. E 85, 021305 (2012).

[7] E. Aharonov and D. Sparks, Phys. Rev. E 60, 6890 (1999).

[8] M. Otsuki and H. Hayakawa, Phys. Rev. E 83, 051301 (2011).

[9] M. Otsuki and H. Hayakawa, Phys. Rev. E 80, 011308 (2009).

[10] M. P. Ciamarra, R. Pastore, M. Nicodemi, and A. Coniglio, Phys. Rev. E 84, 041308 (2011).

[11] D. Bi, J. Zhang, B. Chakraborty, and R. Behringer, Nature (London) 480, 355 (2011).

[12] The variation of $\mu$ has been studied by Otsuki et al. (see Figs. 9 and 15 and the related discussion in [8]). Near $\mu=2$ the rheological properties depend weakly on this parameter and $\mu=2$ can be regarded as the limit of large friction.
[13] A. Lees and S. Edwards, J. Phys. C: Solid State Phys. 5, 1921 (1972).

[14] See Supplemental Material at http://link.aps.org/supplemental/ 10.1103/PhysRevE.89.050201 for details of the estimation of the packing fractions and further information about the model.

[15] P. D. Olmsted, Rheol. Acta 47, 283 (2008).

[16] C. B. Holmes, M. E. Cates, M. Fuchs, and P. Sollich, J. Rheol. 49, 237 (2005)

[17] P. Olsson and S. Teitel, Phys. Rev. Lett. 109, 108001 (2012).

[18] B. P. Tighe, E. Woldhuis, J. J. C. Remmers, W. van Saarloos, and M. van Hecke, Phys. Rev. Lett. 105, 088303 (2010).

[19] J. Dhont and W. Briels, Rheol. Acta 47, 257 (2008).

[20] M. E. Cates, D. A. Head, and A. Ajdari, Phys. Rev. E 66, 025202 (2002).

[21] H. Nakanishi, S.-i. Nagahiro, and N. Mitarai, Phys. Rev. E 85, 011401 (2012).

[22] The actual transition ("binodal") has to lie somewhere in the shaded region. Its location cannot be constructed within the current model (see [19]).

[23] C. Heussinger and J.-L. Barrat, Phys. Rev. Lett. 102, 218303 (2009). 


\title{
Supplemental Material: Jamming of Frictional Particles: a Non-equilibrium First Order Phase Transition
}

\author{
Matthias Grob, ${ }^{1}$ Claus Heussinger, ${ }^{2}$ and Annette Zippelius ${ }^{1,2}$ \\ ${ }^{1}$ Max Planck Institute for Dynamics and Self-Organization, Am Faßberg 17, 37073 Göttingen, Germany \\ ${ }^{2}$ Institute for Theoretical Physics, Georg-August University of Göttingen, \\ Friedrich-Hund Platz 1, 3707ry Göttingen, Germany
}

(Dated: April 17, 2014)

\section{ESTIMATION OF THE PACKING FRACTION $\left(\varphi_{c}, \varphi_{\sigma}, \varphi_{\eta}\right)$}

This section describes the estimation of the packing fractions $\left(\varphi_{c}, \varphi_{\sigma}, \varphi_{\eta}\right)$ by molecular dynamics data. The flow curves in the main article Fig. 1 show a jump in stress for $\varphi=0.795$ and smooth behaviour for $\phi=$ 0.7925. Therefore we conclude that $\phi_{c}$ lies in between these two values. This is furthermore in agreement with Fig. 4 in the main article where the first solid states $\left(\tau_{\text {jam }}<T\right)$ occur at $\varphi=0.7925$.

The values of $\varphi_{\sigma}$ and $\phi_{\eta}$ are determined as described in the manuscript. The data is displayed in Fig 1.

The precise values for the three characteristic packing fractions are not essential for this work. What is more important is that there are three different packing fractions - and not just one as for the frictionless case. This conclusion is robust and does not rely on the power-law fits.

\section{FURTHER INFORMATION ABOUT THE SCHEMATIC MODEL}

This section puts emphasize on the packing fractions $\left(\varphi_{c}, \varphi_{\sigma}, \varphi_{\eta}\right)$ in the framework of the simple model and aims to provide a better understanding of the phase diagram (schematic) in Fig. 5 in the main article. Fig. 2 is a log-linear representation of the flow curves shown in the main article Fig. 1. The black line is subcritical
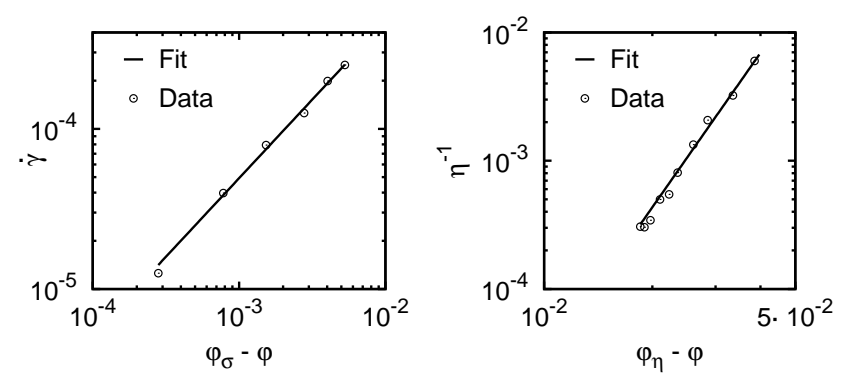

FIG. 1: Left: Fit of the strain rate $\dot{\gamma}_{\sigma}$, where the plastic stress in the upper branch drops to zero to $\dot{\gamma}_{\sigma} \propto\left(\varphi_{\sigma}-\varphi\right)^{\alpha}$, yielding $\varphi_{\sigma}=0.8003(2)$ and $\alpha=1.0(1)$. Right: Fit of the inverse generalized viscosity to $\eta^{-1} \propto\left(\varphi_{\eta}-\varphi\right)^{4}$ yielding $\varphi_{\eta}=$ $0.819(1)$.

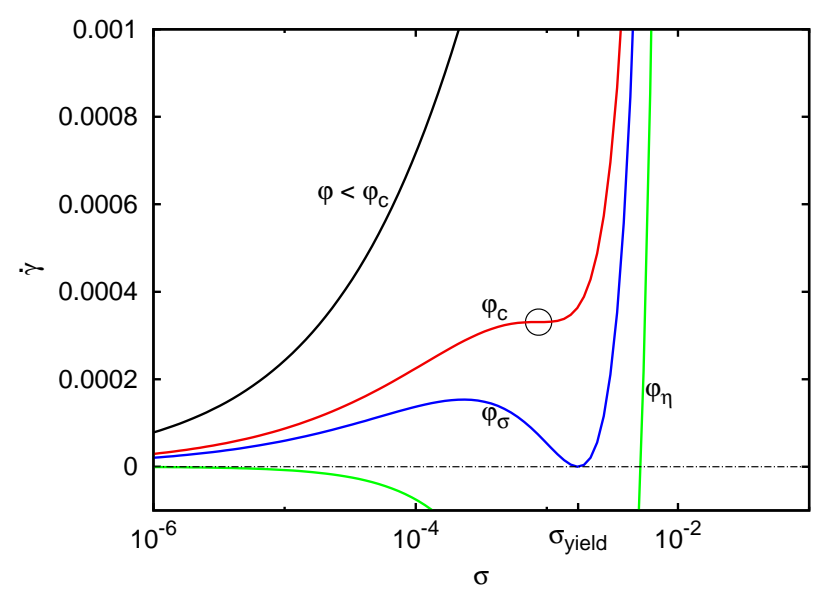

FIG. 2: Flow curves of the simple model Eq. 1 of the main article in log-linear representation. Frictional scenario; the black line is subcritical, $\varphi_{c}$ is indicated by the red line, $\varphi_{\sigma}$ by the blue line and $\varphi_{\eta}$ by the green line. The critical point $\left(\varphi_{c}, \dot{\gamma}_{c}, \sigma_{c}\right)$ is encircled. $\sigma_{\text {yield }}$ indicates the first yield stress, i.e. $\dot{\gamma}=0$ for $\sigma \neq 0$ at $\varphi_{\sigma}$.

and crosses smoothly from plastic to inertial flow as the strain rate is decreased. Upon an increase in packing fraction, $\varphi_{c}$ is the first flow curve with zero slope (analog to the critical isotherm in classical theory of first order phase transition for a fluid-gas-system). At $\varphi_{\sigma}$ the zeroline $(\dot{\gamma}=0)$ is touched at finite stress and at $\varphi_{\eta}$ the coefficient $a=0$ which makes the negative (linear) term dominant for $\sigma \rightarrow 0$. Therefore for $\varphi_{\eta}$ and above the flow curves bend downwards at the origin, i.e. the inertial branch is vanished, which is reflected in a yield stress. 
7. Shear rheology of frictional grains

\subsection{Rheological chaos of frictional grains}

Reprinted article with permission from

Grob, Matthias and Zippelius, Annette and Heussinger, Claus

Physical Review E 93030901 (2016)

http://dx.doi.org/10.1103/PhysRevE.93.030901

Copyright (2016) by the American Physical Society. 


\title{
Rheological chaos of frictional grains
}

\author{
Matthias Grob, Annette Zippelius, and Claus Heussinger \\ Institute of Theoretical Physics, Georg-August University of Göttingen, 37073 Göttingen, Germany \\ (Received 28 July 2015; revised manuscript received 24 February 2016; published 14 March 2016)
}

\begin{abstract}
A two-dimensional dense fluid of frictional grains is shown to exhibit time-chaotic, spatially heterogeneous flow in a range of stress values, $\sigma$, chosen in the unstable region of S-shaped flow curves. Stress-controlled simulations reveal a phase diagram with reentrant stationary flow for small and large stress $\sigma$. In between, no steady flow state can be reached, instead the system either jams or displays time-dependent heterogeneous strain rates $\dot{\gamma}(\mathbf{r}, t)$. The results of simulations are in agreement with the stability analysis of a simple hydrodynamic model, coupling stress and microstructure which we tentatively associate with the frictional contact network.
\end{abstract}

DOI: 10.1103/PhysRevE.93.030901

Discontinuous shear thickening is a ubiquitous phenomenon, observed in many dense suspensions [1,2]. Simulations for non-Brownian particle suspensions [3-5] as well as for frictional granular media [6,7] have highlighted the particular role played by frictional particle interactions. Discontinuous shear thickening implies a region of shear stress which (at finite Reynolds number, Ref. [8]) is not accessible to a homogeneous system in stationary state. What happens if the system is forced into this regime by prescribing the stress at the boundary in the unstable region? One possibility is vorticity banding [9], corresponding to bands with different stress values at the same shear rate. However, there is no clear evidence for persistent vorticity banding in experiment so far. Furthermore objections have been raised as to the possibility of vorticity banding as a stationary state: The pressure-in contrast to the shear stress-has to be the same across the interface of the bands; otherwise particle migration is expected to occur and thereby destabilize the interface. If stationary states are not accessible to the system, we expect to observe time-dependent, inhomogeneous states, either oscillatory or chaotic [10].

In this Rapid Communication we show that spatiotemporal chaos occurs in a two-dimensional system of frictional granular particles subject to an applied stress which is chosen in the unstable region of the flow curve. We present results from simulations and formulate a hydrodynamic model to derive a phase diagram and identify the regions of parameter space, where time-chaotic, inhomogeneous solutions are to be found.

We simulate a two-dimensional system of $N$ soft, frictional particles in a square box of linear dimension $L$ as detailed in [7]. The particles all have the same mass $m=1$, but are polydisperse in size with diameters $0.7,0.8,0.9$, and 1 in equal amounts. Normal and tangential forces, $\boldsymbol{f}^{(n)}$ and $f^{(t)}$, are modeled with linear spring-dashpots of unit strength for both elastic and viscous contributions. Thereby units of time, length, and mass have been fixed [11]. Flow curves for other viscoelastic parameters are presented in the Appendix. Coulomb friction is implemented with friction parameter $\mu=$ 2 , corresponding to the high friction limit. We expect the same qualitative findings as presented in this Rapid Communication for all values of $\mu>0$ and refer to a systematic study of the $\mu$ dependence in Ref. [6]. In the stress-controlled simulations, a boundary layer of particles is frozen and the boundary at the top is moved with a force $\sigma L \hat{\boldsymbol{e}}_{x}$, whereas the bottom plate remains at rest. In shear direction we use periodic boundary conditions.

Constitutive equation. Previous work [6,7] has revealed discontinuous shear thickening for a range of packing fractions close to the jamming transition. The flow curves for frictional granular particles are well represented by the following constitutive equation:

$$
\dot{\gamma}(\sigma)=a \sigma^{1 / 2}-b \sigma+c \sigma^{2},
$$

where the term $-b \sigma$ is due to the frictional interactions, and dependence on the packing fraction $\phi$ is implemented with $a=a(\phi)[7,12]$. This gives rise to the phenomenology of the van der Waals theory for a first-order phase transition: jamming first occurs at the critical point $\phi_{c} \cong 0.795$ which also marks the onset of hysteresis. A finite yield stress first appears at $\phi_{\sigma} \cong 0.8003$, while the generalized viscosity, $\eta=\sigma / \dot{\gamma}^{2}$, diverges only at $\phi_{\eta} \cong 0.819$. A similar, but not identical, sequence of characteristic packing fractions has been proposed in $[6,13]$. Most remarkable is the existence (due to the $b$-dependent term) of a regime of shear stress which is unstable $\frac{\partial \dot{\gamma}}{\partial \sigma}<0$, corresponding to s-shaped flow curves. In strain-controlled conditions such an $\mathrm{s}$ shape leads to discontinuous shear thickening. In the van der Waals theory it corresponds to the coexistence region.

Simulations in the unstable regime. These S-shaped flow curves are indeed observed in the simulations (see Fig. 1), however, only as transients and only in rather small simulation cells $(N<24000)$. In larger systems the $s$ shape is slowly eroded and vanishes completely above a certain system size. Instead a regime of continuous shear thickening develops. Closer inspection of the simulations in this regime reveals that no simple steady state is reached. Rather, the system displays time-chaotic and spatially inhomogeneous behavior. An example is shown in Fig. 2, which is a sequence of four snapshots of the local-stress field (movies are given in the Supplemental Material [14]). The system does not settle into a time-independent steady state on the time scale of the simulations. Instead one observes time-dependent large-scale structures, e.g., shear bands which seem to propagate in the principal stress direction, alternating with approximately homogeneous states and random large-scale structures. In Fig. 5 we show the corresponding time-dependent strain rate. One clearly observes irregular time dependence with intermittent oscillatory periods. 


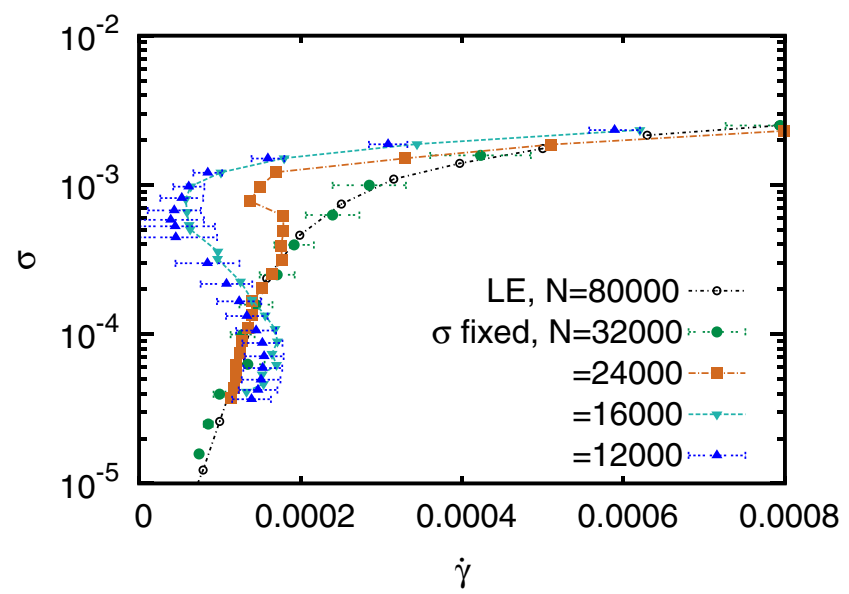

FIG. 1. Evolution of flow curves $\sigma(\dot{\gamma})$ with system size $N$. For small systems a pronounced $\mathbf{s}$ shape is visible in transients before the system jams. In large systems no steady flow occurs; average over the time-dependent flow results in continuous shear thickening (stress controlled except for $N=80000$ ).

Hydrodynamic model. To understand these time-dependent solutions and locate the regions of parameter space where they can occur, we now formulate a hydrodynamic model, determine its stationary states, and analyze their stability. Our starting point is the momentum conservation equation in the form $\partial_{t} v_{x}=\partial_{y} \sigma_{x y}$. For simplicity we only consider a one-dimensional model, allowing for a velocity $v_{x}$ in the flow direction, dependent on $y$ only. In addition we introduce a variable $w(y, t)$ for the internal state or the microstructure of the fluid. Such a variable has been introduced for many complex fluids, such as liquid crystals, entangled polymer solutions, and colloidal systems [9]. Here we associate it with the frictional contact network. In the simplest model we only consider a scalar variable, representing, e.g., the number of frictional contacts $[8,15]$, but are aware that a tensorial quantity, such as the fabric tensor, might be more appropriate. We assume that the microstructure variable
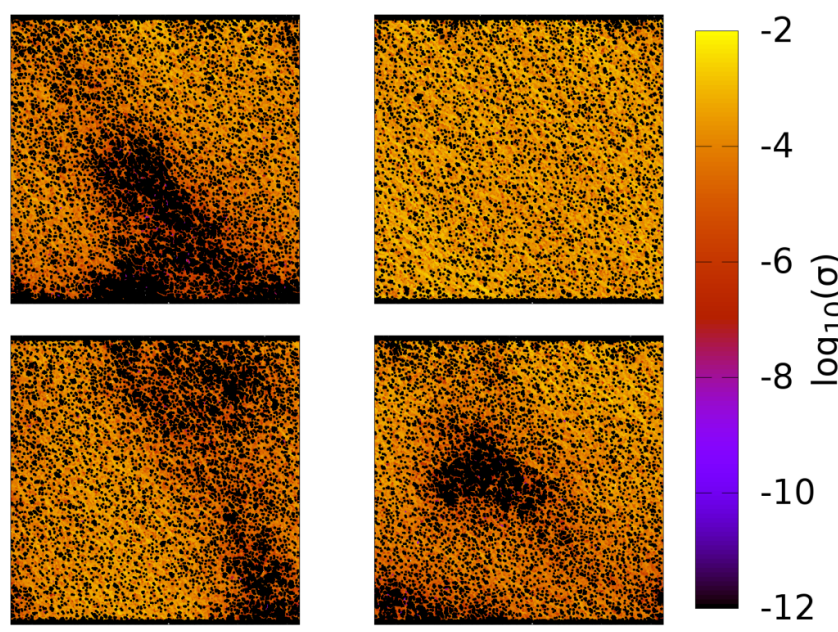

FIG. 2. Four snapshots of the local stress, revealing large-scale, time-dependent structures; $\phi=0.8035$. relaxes to a stationary state $\partial_{t} w=\left(w-w^{*}\right) / \tau$ which should vanish in the absence of stress $w^{*}(\sigma \rightarrow 0) \rightarrow 0$. Furthermore dynamic rearrangements occur only due to driving, so that $\tau^{-1} \propto \dot{\gamma}$. So far the model is the same as considered by Nakanishi et al. [16] for dilatant fluids. However, the coupling of stress and microstructure is different for the frictional grains under consideration. The frictional contacts reduce the flow and hence the velocity gradient. Starting from the constitutive equation for the strain rate of frictionless grains $\dot{\gamma}_{0}=a \sigma^{1 / 2}+c \sigma^{2}$, we take the strain rate of our system of particles with friction to be $\dot{\gamma}=\dot{\gamma}_{0}-w$. This completes the definition of the hydrodynamic model

$$
\begin{aligned}
\partial_{t} \dot{\gamma} & =\partial_{y}^{2} \sigma, \\
\dot{\gamma} & =\dot{\gamma}_{0}-w, \\
\partial_{t} w & =-\frac{\dot{\gamma}}{\Gamma}\left(w-w^{*}\right) .
\end{aligned}
$$

Here we have introduced a proportionality constant, $\tau=\Gamma / \dot{\gamma}$, which can be fitted, when comparing simulations to the predictions of the model. For the stability analysis, it is irrelevant. Higher-order diffusive terms may be added to the stress and/or the $w$ equation. We have checked that the inclusion of such terms does not change the stability analysis. Other hydrodynamic models of granular fluids include velocity fluctuations [17], e.g., granular temperature which, however, cannot explain effects due to friction.

The model allows for two stationary states: The first one corresponds to stationary flow and is explicitly given by

$$
\dot{\gamma}=\dot{\gamma}_{0}-w^{*}, \quad w=w^{*}, \quad \sigma=\sigma_{0} .
$$

Given that $w^{*}$ should vanish for vanishing shear, we take it as $w^{*}=b \sigma$, so that we recover the constitutive relation for frictional grains, Eq. (1). The second stationary solution accounts for the jammed state and reads

$$
\dot{\gamma}=0, \quad w=\dot{\gamma}_{0}, \quad \sigma=\sigma_{0} .
$$

For both stationary states, the stress $\sigma=\sigma_{0}$ is homogeneous over the sample, in agreement with the Navier-Stokes equation which require a homogeneous stress in two dimensions and hence does not allow vorticity banding.

Stability analysis. To study the stability of the stationary states, we consider small deviations $\delta \sigma, \delta w \sim e^{\Omega t} e^{i k y}$ and linearize Eq. (2) in $\delta \sigma, \delta w$. As expected the stationary flow is unstable for $\frac{\partial \dot{\gamma}}{\partial \sigma}<0$. Below $\phi_{c}$, this does not occur and we find two stable modes: a hydrodynamic one, $\Omega_{1} \propto-k^{2}$, corresponding to the conservation of momentum, and a nonhydrodynamic one, $\Omega_{2} \propto-\frac{\partial \dot{\gamma}}{\partial \sigma} \rightarrow 0$, corresponding to the relaxation of the microstructure, whose relaxation time becomes infinite as $\phi \rightarrow \phi_{c}$ and $\sigma \rightarrow \sigma_{c}$. Above $\phi_{c}$, the model predicts two stable stationary flow solutions: inertial flow at small stress and plastic flow at large stress. In between, a gap of unstable stress values occurs such that no stationary homogeneous flow is possible in this range of stresses. A typical flow curve in the range $\phi_{c}<\phi<\phi_{\sigma}$ is shown in the inset of Fig. 3 as line 1, indicating the unstable regime as red. The jammed state is only stable for $\phi>\phi_{\sigma}$ in the region where the constitutive relation yields a negative $\dot{\gamma}$. A typical flow curve in the range $\phi_{\sigma}<\phi<\phi_{\eta}$ is line 2 in the inset of Fig. 3 with the stable jammed state marked in blue. For 


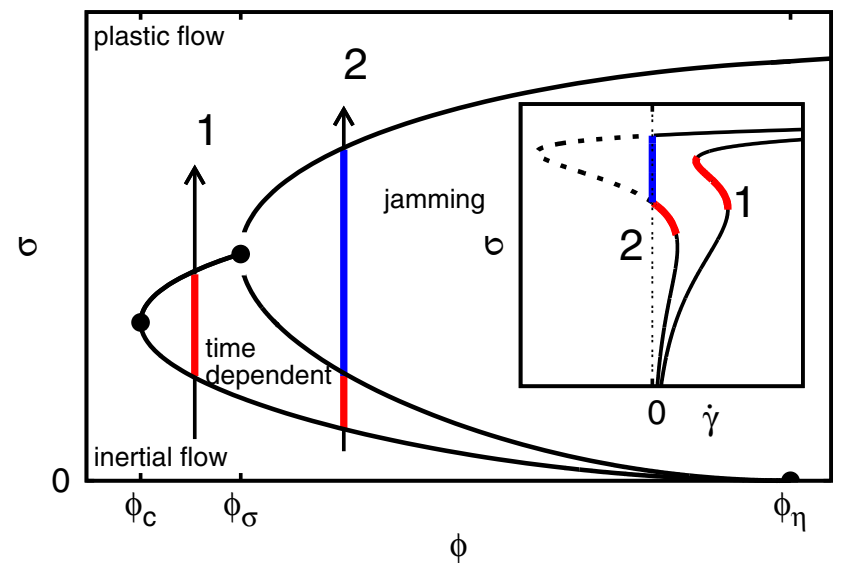

FIG. 3. Inset: Typical stationary states (flow curves) in the range $\phi_{c}<\phi<\phi_{\sigma}$ (1) and $\phi_{\sigma}<\phi<\phi_{\eta}$ (2). Unstable regions are highlighted in red (time-dependent flow) and blue (jamming). Main: Phase diagram following from the linear stability analysis; the two generic flow curves, displayed in the inset, correspond to the paths, denoted by 1 and 2 .

$\phi>\phi_{\eta}$, only stationary plastic flow and stationary jamming are predicted by linear stability analysis. The resulting phase diagram of the model is shown in the main panel of Fig. 3.

Above the critical packing fraction, $\phi_{c}$, a finite range of inaccessible stress values with $\frac{\partial \dot{\gamma}}{\partial \sigma} \leqslant 0$ exists and gives rise to a corresponding range of unstable wave numbers $k^{2} \leqslant k_{c}^{2}=\left|\dot{\gamma} \frac{\partial \dot{\gamma}}{\partial \sigma}\right|$, which shrinks to 0 as $\phi \rightarrow \phi_{c}$. Hence the model predicts an approximately harmonically oscillating state at the onset of instability, while well inside the unstable region more and more wave numbers are unstable so that one expects a broad range of frequencies to be present in the spectrum. These expectations are born out by numerical integration of the partial differential equations in order to obtain the full nonlinear dynamical evolution. Close to $\phi_{c}$, the oscillations are approximately harmonic, while we find oscillating and seemingly chaotic solutions at larger packing fractions (see Fig. 4).

Comparison with simulations. To check the predictions of the above analysis, we performed stress-controlled simulations (for technical details see Ref. [7]) along paths 1 and 2 in the phase diagram. The time-dependent strain rate along path 1 is

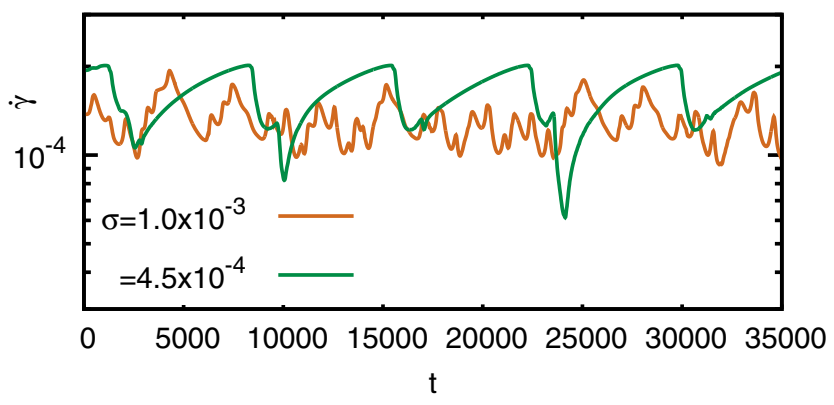

FIG. 4. Spatially averaged strain rate vs time from numerically integrating the hydrodynamical model for different imposed stress. In the unstable region at intermediate stress values, we observe oscillations and chaotic solutions $\left(\Gamma=10^{-3}, \phi=0.7975\right)$.

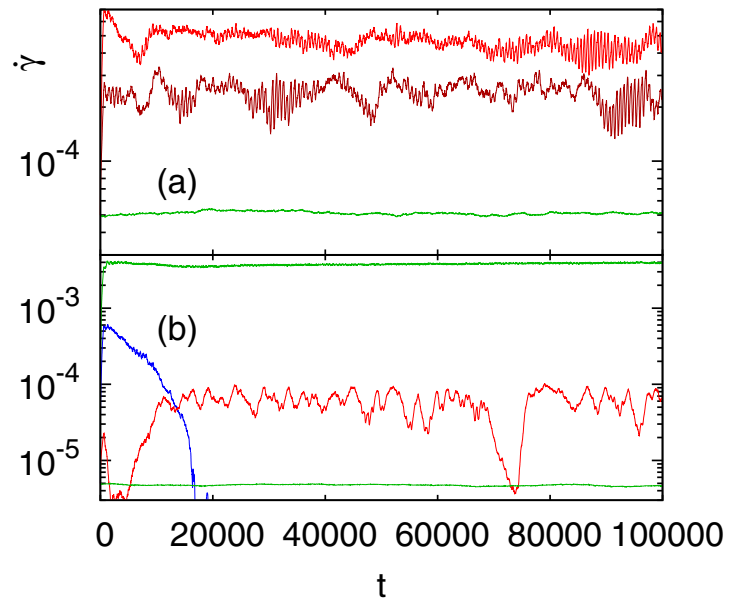

FIG. 5. Strain rate $\dot{\gamma}(t)$ as a function of time for several values of stress, corresponding to path 1 (top, $\phi=0.7975$ ) and path 2 (bottom, $\phi=0.8035$ ) in Fig. 3; the sudden drops of the strain rate in the timedependent flow curve indicate that the system nearly gets jammed.

shown in Fig. 5(a) for three different stress values. The lowest one shows stationary flow in the Bagnold regime, the chaotic time dependence with oscillatory components is represented by the two red curves for intermediate stress, and larger stress gives rise to stationary plastic flow [not shown in Fig. 5(a)]. Similarly in Fig. 5(b) we show the strain rate as a function of time for path 2 . In addition to the steady-state flow, and the oscillatory flow, there are stationary jammed states, where the initial flow ceases after a certain amount of time.

To get a quantitative measure of the irregularity in the time dependence of the states, we have computed the power spectrum $C(\omega)$ as the Fourier transform of the strain rate autocorrelation function (Fig. 6). In the stable regimes, the power spectrum is $C \sim \omega^{-2}$, suggesting simple exponential correlations and linear noise. In the unstable regime this background spectrum is superposed by additional and irregular complex structures. This is a strong indication of truly nonlinear chaotic dynamics. Noteworthy is also the strong peak at higher stresses. This corresponds to the fast oscillations visible in Fig. 5(a).

Finite-size effects. The snapshots in Fig. 2 indicate the buildup of large-scale coherent structures. A large correlation length has also been observed in Ref. [3] in the context of continuous shear thickening in non-Brownian particle suspensions. These correlations are also in line with the strong finite-size effects observed in the flow curves of Fig. 1. Indeed, in lowering the system size, the oscillatory state acquires a finite lifetime and the system jams. This is because in small systems there is a finite probability that large strain rate fluctuations towards $\dot{\gamma} \rightarrow 0$ lead to a stable jammed state [see Fig. 5(b) for an example of such an excursion]. Similarly, the appearance of S-shaped flow curves is due to system-size-dependent strong strain rate fluctuations towards the jammed state with $\dot{\gamma}=0$.

Conclusion. We discuss stress-controlled driving of a granular system that undergoes discontinuous shear thickening. In particular a regime is identified where the system does not settle into a time-independent steady state. Instead, it 


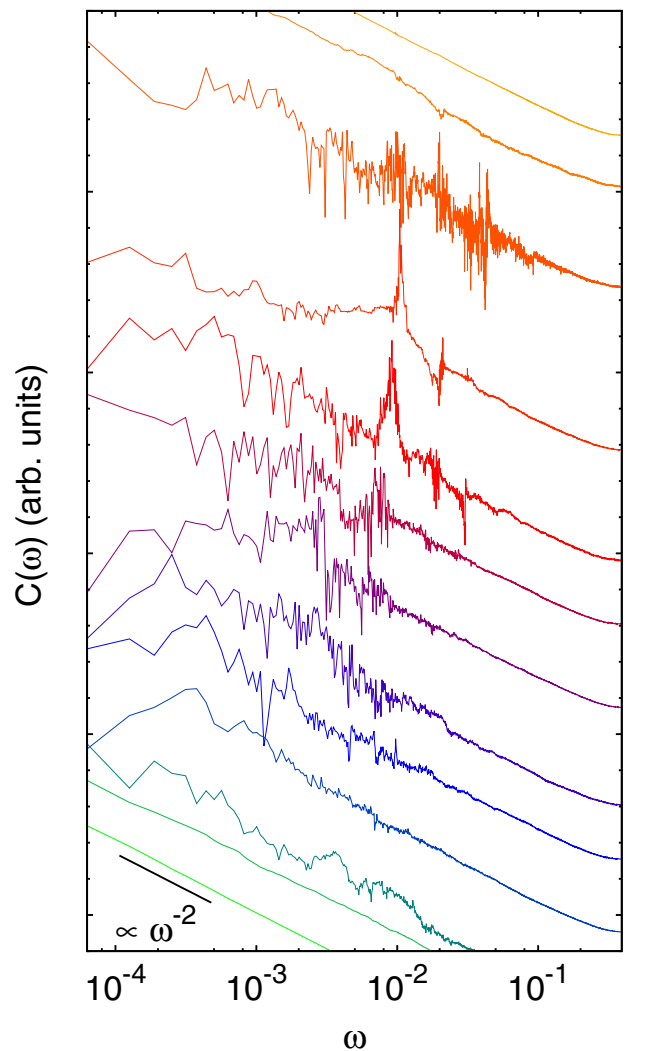

FIG. 6. Power spectrum $C(\omega)$ of strain rate fluctuations at constant stress from low (bottom) to high (top) values of $\sigma$ (shifted vertically for clarity of presentation); in the stable region $C \sim \omega^{-2}$; whereas in the unstable region additional complex structures are superimposed on the $\omega^{-2}$ decay.

displays spatiotemporal oscillations and chaotic behavior as a novel possibility to adopt to stress in the unstable parts of the flow curve. Recent experiments on corn starch reveal very similar unsteady flow where theory predicts discontinuous shear thickening [18].

Simulations reveal a phase diagram that has a characteristic re-entrant form with steady-state flow at small and large stresses. At intermediate values of stress either time-dependent states are observed or the system settles into a nonflowing jammed state, depending on stress and packing fraction. For $\phi_{c}<\phi<\phi_{\sigma}$ only time-dependent solutions are observed, whereas for $\phi_{\sigma}<\phi$ a sequence of inertial flow, chaotic flow, jammed state, and plastic flow are seen for increasing values of $\sigma$, until at $\phi=\phi_{\eta}$ only a transition from the jammed state to plastic flow remains.

We also present a hydrodynamical model, coupling stress to a microstructural observable. Within linear stability analysis we recover the detailed features of the phase diagram as obtained from simulations. In the unstable region, the model predicts either oscillating or time-chaotic flow.

In future work we plan to quantify the spatiotemporal correlations that are visible in the snapshots and compare them with length scales determined in [3] from velocity correlations. We furthermore aim to better understand the microstructural observable, check whether it can be associated
TABLE I. Normal restitution coefficient $\epsilon^{(n)}$ and binary collision time $t$ for different $k$ and $\eta$.

\begin{tabular}{lcll}
\hline \hline$k$ & $\eta$ & $\epsilon^{(n)}$ & $t$ \\
\hline 1 & 1 & 0.305 & 2.375 \\
1 & $1 / 2$ & 0.569 & 2.26 \\
1 & $1 / 10$ & 0.895 & 2.22 \\
$1 / 2$ & 1 & 0.163 & 3.63 \\
2 & 1 & 0.44 & 1.4 \\
10 & 1 & 0.7 & 0.7 \\
\hline \hline
\end{tabular}

with the contacts which are blocked by Coulomb friction, and explore the possibility of a tensorial observable, such as the fabric tensor.

Acknowledgments. We gratefully acknowledge financial support by the Deutsche Forschungsgemeinschaft via programs FOR 1394 and Emmy Noether He 6322/1-1.

\section{APPENDIX}

In this Appendix we deal with flow curves of frictional grains at fixed packing fraction when the particles' elastic and viscous damping constants change. In the main text we use unit strength for elastic and viscous contributions in the linear-spring dashpots ( $k$ and $\eta$, respectively). Units of time and energy dissipation when particles interact are set by this choice. In particular, the coefficient of (normal) restitution, $\epsilon^{(n)}$, and the binary collision time, $t$, are set. Table I summarizes the normal coefficient of restitution and the binary collision time for the parameters that we discuss here. We tune both contributions, $k=k^{(n)}=k^{(t)}$ and $\eta=\eta^{(n)}=\eta^{(t)}$, independently. Both $\epsilon^{(n)}$ and $t$ just serve as a guidance on properties of pairwise collisions and do not respect the large packing fraction. Also the important frictional contribution which implies tangential restitution $\epsilon^{(t)}$ is not characterized by these quantities. The normal restitution can be computed easily while the tangential part is of rich and complicated behavior due to its dependence on the impact velocities [19].

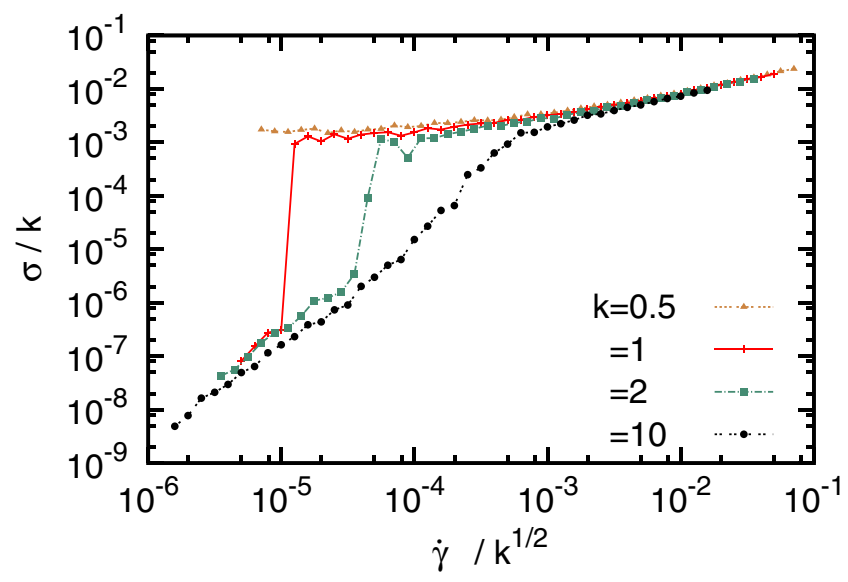

FIG. 7. Scaled flow curves for $\phi=0.80$, viscous damping parameter $\eta=1$ and varying elastic constant $k$. 


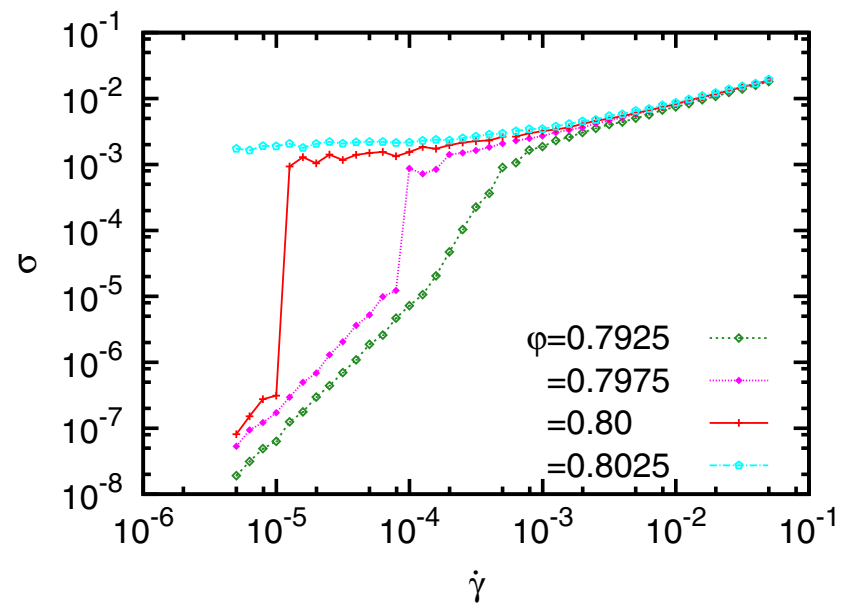

FIG. 8. Flow curves for varying packing fraction $\phi$ across the transition with viscous damping parameter $\eta=1$ and elastic constant $k=1$.

The following data show flow curves of a system with $N=8000$ particles. Figure 7 shows scaled flow curves for fixed $\phi=0.80$ and $\eta=1$. When $k$ decreases $\left(\epsilon^{(n)}\right.$ decreases), the discontinuity shifts towards small strain rate until it is missed out by our simulation. Note that the numerical effort for low strain rate is much larger than for large strain rate. An increasing $k$ (increasing $\epsilon^{(n)}$ ) shifts the discontinuity towards larger strain rate which makes the discontinuity smaller until it vanishes. At $k=10$ the transition from inertial to plastic flow is smooth and without shear thickening. The phenomenology of varying $k$ seems in that range similar to a change of the packing fraction. The latter was studied in previous work [7].

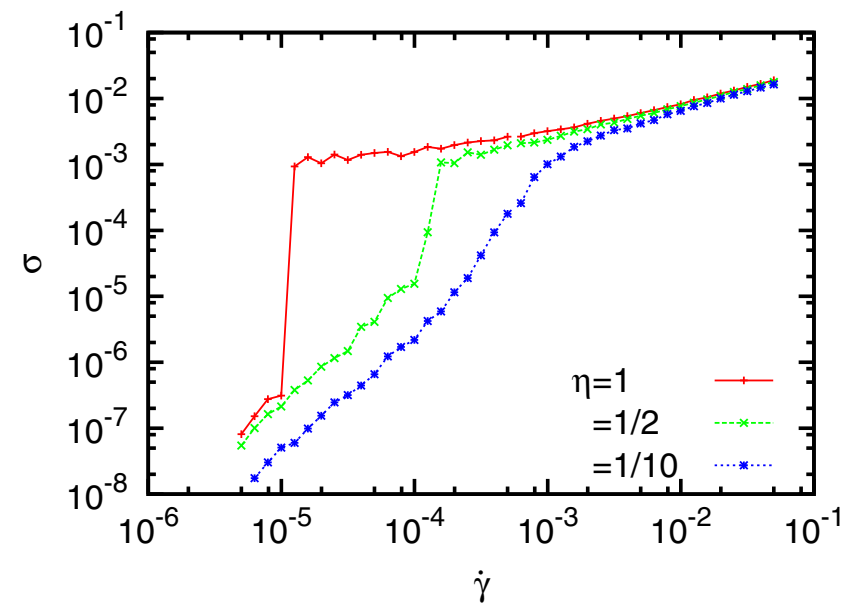

FIG. 9. Flow curves for $\phi=0.80$, elastic constant $k=1$, and varying viscous damping parameter $\eta$.

A systematic study of variations of all three parameters, $\phi, k$, and $\eta$, is out the scope of this article. In Fig. 8 we show a choice of packing fractions with phenomenology similar to Fig. 7. Lower $\phi$ leads to flow curves similar to those with large $\epsilon^{(n)}$, and larger $\phi$ shows the same behavior as small $\epsilon^{(n)}$. The variation of the viscous damping parameter $\eta$ is shown in Fig. 9. The phenomenology is the same as above: decreasing $\epsilon^{(n)}$ (larger $\eta$ ) leads to a shift towards small strain rates and an increasing coefficient of restitution (smaller $\eta$ ) shifts the discontinuity towards larger strain rate until it vanishes. Then the transition between inertial and plastic flow is smooth and without shear thickening again.
[1] E. Brown and H. M. Jäger, Rep. Prog. Phys. 77, 046602 (2014).

[2] Z. Pan, H. de Cagny, B. Weber, and D. Bonn, Phys. Rev. E 92, 032202 (2015).

[3] C. Heussinger, Phys Rev. E 88, 050201(R) (2013).

[4] R. Seto, R. Mari, J. F. Morris, and M. M. Denn, Phys. Rev. Lett. 111, 218301 (2013).

[5] N. Fernandez, R. Mani, D. Rinaldi, D. Kadau, M. Mosquet, H. Lombois-Burger, J. Cayer-Barrioz, H. J. Herrmann, N. D. Spencer, and L. Isa, Phys. Rev. Lett. 111, 108301 (2013).

[6] M. Otsuki and H. Hayakawa, Phys. Rev. E 83, 051301 (2011).

[7] M. Grob, C. Heussinger, and A. Zippelius, Phys. Rev. E 89, 050201(R) (2014).

[8] R. Mari, R. Seto, J. F. Morris, and M. M. Denn, Phys. Rev. E 91, 052302 (2015).

[9] P. Olmsted, Rheol. Acta 47, 283 (2008).

[10] M. E. Cates, D. A. Head, and A. Ajdari, Phys. Rev. E 66, 025202(R) (2002).

[11] This leads to a normal coefficient of restitution of $\epsilon^{(n)} \cong 0.3$. Due to the frictional interaction the full coefficient of restitution cannot be evaluated analytically. For a discussion, see J. Schäfer, S. Dippel, and D. E. Wolf, J. Phys. I 6, 5 (1996).

[12] These values correspond to $a=a_{0}\left|\phi_{\eta}-\phi\right|\left(\phi_{\eta}-\phi\right), a_{0}=$ 52.05, $c=147.27$, and $b=\frac{3}{2} a\left(\phi_{c}\right)^{2 / 3} c^{1 / 3}$.

[13] M. P. Ciamarra, R. Pastore, M. Nicodemi, and A. Coniglio, Phys. Rev. E 84, 041308 (2011).

[14] See Supplemental Material at http://link.aps.org/supplemental/ 10.1103/PhysRevE.93.030901 for movies of time-dependent flow.

[15] M. Wyart and M. E. Cates, Phys. Rev. Lett. 112, 098302 (2014).

[16] H. Nakanishi, S. I. Nagahiro, and N. Mitarai, Phys. Rev. E 85, 011401 (2012).

[17] N. V. Brilliantov and T. Pöschel, Kinetic Theory of Granular Gases (Oxford University, Oxford, 2004).

[18] M. Hermes, B. M. Guy, G. Poy, M. E. Cates, M. Wyart, and W. C. Poon, arXiv:1511.08011.

[19] V. Becker, T. Schwager, and T. Pöschel, Phys. Rev. E 77, 011304 (2008). 
7. Shear rheology of frictional grains

7.3. Unsteady rheology and heterogeneous flow of dry frictional grains 


\title{
Unsteady rheology and heterogeneous flow of dry frictional grains
}

\author{
Matthias Grob, Claus Heussinger, and Annette Zippelius \\ Institute of Theoretical Physics, Georg-August University of Göttingen, 37073 Göttingen, Germany
}

(Dated: June 27, 2016)

\begin{abstract}
Using molecular dynamics simulations we investigate heterogeneous and unsteady states in shear thickening frictional granular media. We characterize the heterogeneous states by the decay of the stress-stress correlation function providing a characteristic length scale. In only large simulation cells, in which shear thickening is continuous, both, heterogeneities and unsteady states, are shown to exist. The analysis of stress and strain controlled simulations in differently sized simulation cells explains the role played by a length that is considerably larger than the particle size. Simulations of small simulations cells relate shear jamming to discontinuous shear thickening. Local measures, e.g., the nonaffine velocity or the local coordination number, are shown to follow instantaneously the evolution of stress.
\end{abstract}

\section{INTRODUCTION}

Flow properties of dense granular matter are of fundamental importance in a wide range of engineering disciplines and nature. Many industrial resources are shipped and processed as grains. In nature, sand is one of the most abundant materials on earth. Collections of grains can also attain solid states when they jam into unordered and rigid states at large packing fractions. The transition from fluid to solid is called jamming. Granular matter is considered athermal as the energy associated with motion or contact is by orders of magnitude above the thermal energy $[1,2]$. The phase diagram for athermal granular media shows that the packing density and the shear stress control whether arrest or flow is achieved [3]. Arrest and flow are separated by the yield stress line, which goes to zero stress, $\Sigma=0$, at a packing fraction, $\phi_{\mathrm{J}}$, called jamming point. For frictionless spheres - spheres which interact only along the normal direction at the contact - flow and arrest around the jamming point have been investigated thoroughly [4].

However, interactions of grains are typically frictional. Rheometry with suspensions of PMMA and silica spheres highlight the role of frictional contacts [5-7]. Experiments with photoelastic discs show that jamming is possible below the isotropic jamming point when a finite shear stress is applied [8]. Corn starch experiments show a shear induced rigidity transition that is either shear thickening or shear jamming $[9,10]$. In computer simulations friction is controlled parametrically, thereby allowing for a direct comparison to the frictionless scenario. Numerical investigations show that $\phi_{\mathrm{J}}$ loses its exceptional role in the characterization of the rheology close to the jamming transition. Instead, more than one packing fraction is necessary for a thorough description of flow and jamming [11-14]. Moreover, simulations with frictional spheres exhibit novel phenomena in the dense flow regime, e.g., transient flow that ceases after a characteristic time $[11,13]$, hysteresis in the flow curve $[12,13]$, the coexistence between a low viscosity and a high viscosity state [15], or even rheo-chaotic response [14]. The latter is also found in corn starch experiments [16]. Thus, friction influences the dense flow close to jamming fun- damentally.

A remarkable phenomenon in frictional systems is shear thickening, i.e., the growth of viscosity with shear rate or shear stress. Shear thickening can be continuous or discontinuous. Increasing the packing fraction can lead from a continuous shear thickening scenario to a discontinuous shear thickening transition and finally to (shear) jamming $[17,18]$. Flow curves of shear thickening solutions include regions that are mechanically unstable and not accessible by homogeneous flow [19]. Moreover, heterogeneous steady states are not possible due to particle migration [16]. Thus, the only remaining flow scenario is heterogeneous and unsteady flow.

In this article, we perform numerical simulations to study heterogeneous flowing states in dry granular systems. These states lead to unsteady flow and continuous shear thickening. The heterogeneous states possess a typical length scale which rules out heterogeneous flow in small systems. Instead, small systems shear thicken discontinuously. The discontinuous shear thickening in small systems appears across an interval of stress. This interval of stress is identified as unstable. Unstable values of stress lead to unsteady flow in large systems contrasting shear jamming in small systems. The overall dynamics is not reflected only in the shear stress but also in other (local) measures which adopt to the flowing state, either stationary or unsteady.

The article is organized as follows: First, we recapitulate in sec. II the description of frictional particle flow by means of flow curves. In sec. III, we present the molecular model, our simulation set-up and measured quantities. In sec. IV, we characterize heterogeneous states, show their time dependence. We relate discontinuous shear thickening to shear jamming and continuous shear thickening to time dependent states. Subsequently, we show that the width of the velocity distribution shows non-monotonic behavior and is related to the generalized viscosity. A discussion and conclusion close the article in sec. V. 


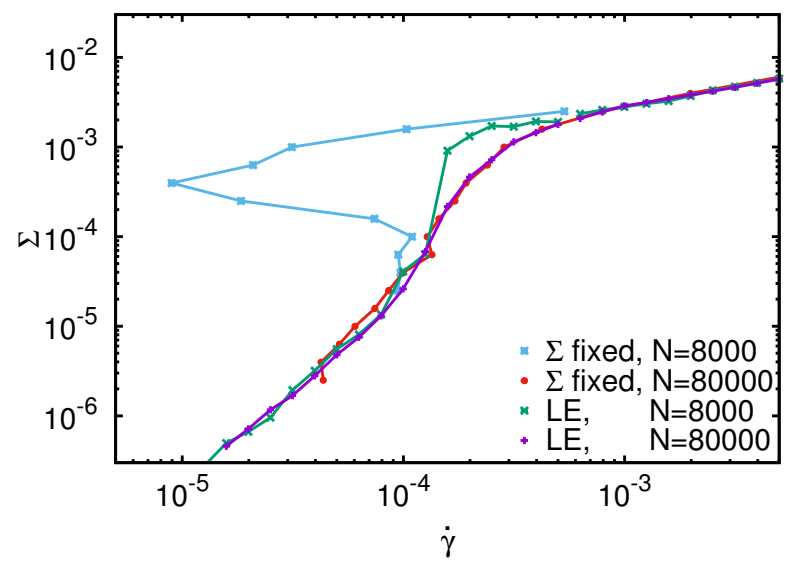

FIG. 1. Flow curve for fixed $\phi=0.7975$ but varying system size. Imposed strain rate indicated by "LE".

\section{FLOW CURVES OF DRY GRANULAR PARTICLES}

Dry granular media show a rich phenomenology when deformed and flow curves (see, e.g., [13, 20, 21]) characterize the flow regimes. Below jamming at low strain rates, inertial flow (also called Bagnold flow) is characterized by a quadratic dependence of the global stress on the strain rate: $\Sigma \propto \dot{\gamma}^{2}$. In this regime the only relevant time scale is the inverse strain rate, $\dot{\gamma}^{-1}$, dominating its counterpart, $\sqrt{k^{-1}}$, set by the spring constant of the particles, $k$, which reflects the particle's stiffness. Bagnold flow corresponds to flow of hard spheres. However, granular particles possess a finite stiffness that becomes relevant at a characteristic strain rate or when the system is dense: plastic flow, either quasistatic ( $\Sigma=$ const.) or rapid $\left(\Sigma \propto \dot{\gamma}^{1 / 2}\right)$, establishes. In previous work [13, 14], the authors suggested s-shaped flow curves which capture these flow regimes. Implications of the s-shape are flow instabilities, which eventually lead to chaotic behavior in large systems [14], or shear jamming in small systems [13]. However, the relation between these phenomena remained elusive.

Figure 1 shows flow curves for differently sized systems and either controlled by strain rate or by shear stress. In the shear rate controlled setting, flow curves show shear thickening. This is either discontinuous, when the stress jumps, or continuous when the stress changes continuously with the strain rate. When the shear stress is controlled and the strain rate is measured, the discontinuous shear thickening turns into an s-shape, which is caused by excursions of the system to nearly jammed states [14]. The unstable stress interval in which no persistent flow is possible is clearly identified in the small system: the stress jumps across an interval of stress when the strain rate is controlled and in this interval we report jammed states when the stress is controlled. The large system's characterization is subtle: irrespective of the protocol, flow curves look well defined but, in fact, the unstable stress interval reveals time dependent heterogeneous flow which is a subject of this article.

\section{MODEL AND METHODS}

\section{A. Molecular model}

The setup in this article is identical to those of former studies $[13,14]$. Due to the brevity of the former articles we give full details of modeling and the simulation here. We consider $N$ soft particles in a square box of linear dimension, $L . N$ and $L$ determine the packing fraction, $\phi=\sum_{i=1}^{N} \pi / 4 d_{i}^{2} / L^{2}$. Forces between particles, $i$ and $j$, arise at contact and are frictional. Frictional contact forces have a normal component, $\mathbf{f}_{i j}^{(\mathrm{n})}$, and a tangential component, $\mathbf{f}_{i j}^{(\mathrm{t})}$. We implement the model by Cundall and Struck [22] with an additional tangential viscous component as done by others, see, e.g., [12, 21, 23-25]. The Coulomb criterion limits the amount of tangential force by a material specific multiple, $\mu$, of the normal force:

$$
\left|\mathbf{f}_{i j}^{(\mathrm{t})}\right| \leq \mu\left|\mathbf{f}_{i j}^{(\mathrm{n})}\right|
$$

$\mu$ is called the friction coefficient. A contact is said to slide if $\left|\mathbf{f}_{i j}^{(\mathrm{t})}\right|=\mu\left|\mathbf{f}_{i j}^{(\mathrm{n})}\right|$.

The normal interaction is a standard visco-elastic model where the overlap, $\delta_{i j}^{(\mathrm{n})}=R_{i}+R_{j}-\left|\mathbf{r}_{i}-\mathbf{r}_{j}\right|>0$, of particles with radii $R_{i}, R_{j}$ at position $\mathbf{r}_{i}, \mathbf{r}_{j}$, determines the interparticle normal force

$$
\mathbf{f}_{i j}^{(\mathrm{n})}=\left(-k^{(\mathrm{n})} \delta_{i j}^{(\mathrm{n})}-\eta^{(\mathrm{n})} / m_{\mathrm{eff}} \dot{\delta}_{i j}^{(\mathrm{n})}\right) \hat{\mathbf{n}}_{i j} .
$$

The unit vector, $\hat{\mathbf{n}}_{i j}$, points from particle $i$ 's to particle $j$ 's center. When $\eta^{(\mathrm{n})}>0$ collisions are inelastic and if contacts are frictionless a coefficient of (normal) restitution can be evaluated analytically: $\epsilon \approx 0.3$ with unit spring and viscous constants.

The non-sliding tangential force is analog to the normal force. Two particles rotate with angular velocities, $\omega_{i}$ and $\omega_{j}$, and move with velocities, $\mathbf{v}_{i}$ and $\mathbf{v}_{j}$, respectively. The relative tangential velocity of spheres in contact is given by the sum of contributions by rotation and translation, $v_{i j}^{\tan }=R_{i} \omega_{i}+R_{j} \omega_{j}+\left(\mathbf{v}_{i}-\mathbf{v}_{j}\right) \hat{\mathbf{t}}_{i j}$, with the tangential vector, $\hat{\mathbf{t}}_{i j}$. The tangential viscous force is given by $-\eta^{(\mathrm{t})} / m_{\mathrm{eff}} v_{i j}^{\mathrm{tan}}$ and a restoring contribution is determined by the integral of $v_{i j}^{\mathrm{tan}}$ but respecting eq. 1 . Explicitly, $f_{i j}^{(\mathrm{t})}=-\eta^{(\mathrm{t})} v_{i j}^{\mathrm{tan}}-k^{(\mathrm{t})} \delta_{i j}^{(\mathrm{t})}$ if $\left|\mathbf{f}_{i j}^{(\mathrm{t})}\right| \leq \mu\left|\mathbf{f}_{i j}^{(\mathrm{n})}\right|$. Where $\int_{t}^{t+\Delta t} v_{i j}^{\tan }\left(t^{\prime}\right) \mathrm{d} t^{\prime}$ is the increment of the tangential displacement, $\delta_{i j}^{(\mathrm{t})}$, during the interval $[t, t+\Delta t]$ when the contact does not slide. If the contact slides, we set $\delta_{i j}^{(\mathrm{t})}=\mu / k^{(\mathrm{t})}\left|\mathbf{f}_{i j}^{(\mathrm{n})}\right|$ and consequently $f_{i j}^{(\mathrm{t})}=k^{(\mathrm{t})} \delta_{i j}^{(\mathrm{t})}$ to satisfy Coulomb's criterion. 

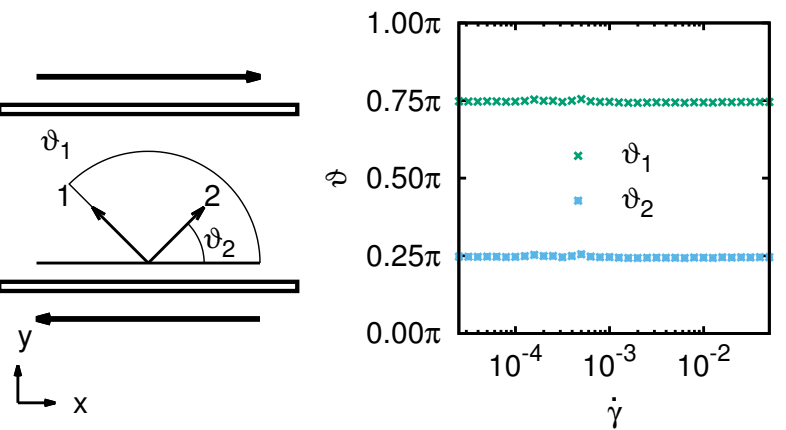

FIG. 2. Left: Simple shear geometry implies a compression and dilation direction with defining angles, $\vartheta_{1 / 2}$. Right: Compression and dilation direction as a function of strain rate $(\phi=0.7975, N=80000)$.

Following previous studies [12-14], four differently sized particle species populate the system with equal amounts and diameters, $d=0.7,0.8,0.9,1.0$, which sets the unit of length. The unit of mass is set by the choice $m=1$. We set $\mu=2$ and we are aware of the comparatively high value of $\mu$, which corresponds to the high friction limit [12].

\section{B. Protocol, geometry and measured quantities}

To control the strain rate we use Lees-Edwards boundary conditions (LE). In stress-controlled simulations we freeze a bottom layer of particles which remains at rest and a boundary layer at the top is moved with a force $\sigma L \mathbf{e}_{x} ;$ periodic boundary conditions are used in shear $(x$-)direction. The geometry is sketched in fig. 2 (left).

Simple shear geometry implies two distinguished directions: compression and dilation direction. These directions are parallel to the eigenvectors of the stress tensor:

$$
\boldsymbol{\Sigma}=\left(\begin{array}{ll}
\Sigma_{x x} & \Sigma_{x y} \\
\Sigma_{y x} & \Sigma_{y y}
\end{array}\right)
$$

with $\Sigma_{\alpha \beta}=1 / N \sum_{i \neq j} r_{i j}^{\alpha} f_{i j}^{\beta}$ and $\mathbf{r}_{i j}=\mathbf{r}_{i}-\mathbf{r}_{j}$. In this paper we use the short hand notation: $\Sigma=\Sigma_{x y}$. The eigenvectors, $\mathbf{v}_{1,2}$, of $\boldsymbol{\Sigma}$ enclose angles, $\vartheta_{1,2}$, with the $x$ axis. In fig. 2 (right) we show that these directions are orthogonal and independent on the strain rate and thus on the flow regime.

In the course of this manuscript we consider correlation functions. To take anisotropy into account we are interested in correlations not only dependent on the distance of particles, $r=|\mathbf{r}|$, but on the displacement vector, $\mathbf{r}=(x, y)$. We compute the correlation of two quantities, $a_{i}$ and $b_{j}$, of particles, $i$ and $j$, as:

$$
C_{a b}(\mathbf{r}=(x, y))=\frac{1}{N} \sum_{i \neq j} a_{i} b_{j} \delta\left(x_{i j}-x\right) \delta\left(y_{i j}-y\right) .
$$

As correlation functions along directions $\mathbf{v}_{1,2}$ we compute:

$$
C_{a b}^{1,2}(r)=\frac{1}{N} \sum_{i \neq j} a_{i} b_{j} \delta\left(x_{i j}-r \cos \vartheta_{1,2}\right) \delta\left(y_{i j}-r \sin \vartheta_{1,2}\right) .
$$

Averages are distinguished between spatial and temporal averages. The spatial average of a quantity, $x$, over a sample with $N$ particles reads:

$$
\langle x\rangle_{N}=\frac{1}{N} \sum_{i=1}^{N} x_{i}
$$

The corresponding standard deviation is defined as $\Delta_{N} x=\sqrt{\left\langle x^{2}\right\rangle_{N}-\langle x\rangle_{N}^{2}}$. The temporal mean is defined with $N_{T}$ measurements at $t_{s}$ with $s \in\left\{1, \ldots, N_{T}\right\}$ as

$$
\langle x\rangle_{T}=\frac{1}{N_{T}} \sum_{s=1}^{N_{T}} x\left(t_{s}\right) .
$$

The corresponding standard deviation is defined as $\Delta_{T} x=\sqrt{\left\langle x^{2}\right\rangle_{T}-\langle x\rangle_{T}^{2}}$.

\section{RESULTS}

\section{A. Heterogeneous time dependent states}

In this section, we study heterogeneous flow states that we observe in large simulation cells in the regime of continuous shear thickening. We explain that the heterogeneous flow states are time dependent as we expect due to the presence of strong gradients in the stress fields. We focus on the evolution of the stress field and on the global stress time series.

Here, we investigate properties of states in which we find characteristics of both, inertial and plastic flow, at the same time. Patches of a typical size show signatures of either inertial or plastic flow. We focus on measurements of local properties, e.g., the stress of a particle $i$, $\sigma_{i}=\sum_{j \neq i} r_{i j}^{x} f_{i j}^{y}$. Other indicators are the local coordination number and the local nonaffine velocity as shown in appendix A. Large systems $(N>24000)$ with intermediate average stress show well separated patches differing by orders of magnitude in the absolute value of local shear stress, see fig. 3. There are well visible stress bands in compression direction that do not percolate in dilation direction. Remarkably, not everywhere do these stress bands span the system in compression direction. In dilation direction the shear stress changes dramatically while we see only weak variations in compression direction. Thus, we can distinguish clearly between compression and dilation direction by visual inspection. 


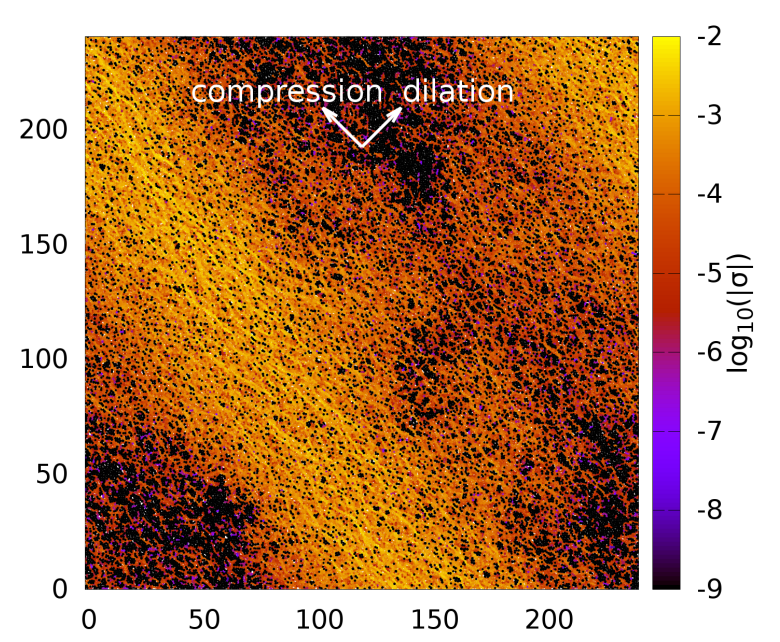

FIG. 3. Snapshot of local stress shows heterogeneous states. $\phi=0.7975$ and $N=80000(L \approx 240)$ with strain rate of $\dot{\gamma}=$ $1.25 \times 10^{-4}$. Particles with zero stress are colored according to the lowest nonzero value that is depicted.
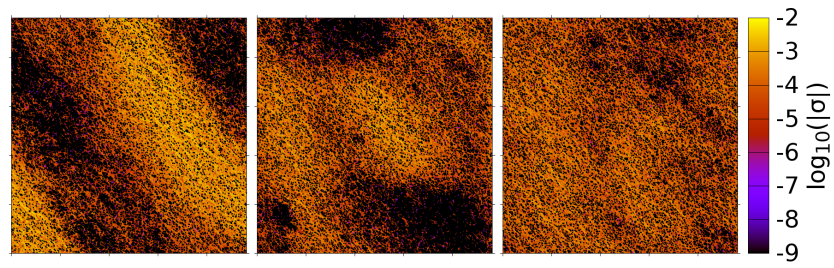

FIG. 4. Time evolution of local stress with same parameters as in fig. 3. Time difference $\Delta t \approx 0.2 \dot{\gamma}^{-1}$.

In the snapshot, fig. 3, the local stress differs by orders of magnitude. These strong stress gradients lead to flow/deformation, i.e., the snapshot shown above is a realization of a time dependent state. The sequence of snapshots in fig. 4 shows the evolution in time (for movies, see the supplemental material to [14]). The stress band that we see in fig. 3 wanders in dilation direction (fig.4 left), eventually interacts with other forming stress bands (fig. 4 middle) and vanishes. Sequences of almost homogeneous states (fig. 4 right) follow before new heterogeneities emerge. Stress bands form again, wander in dilation direction and the dynamics repeats.

The dynamics of the flowing state leads to a time dependent stress on the particles, $\sigma$. This is reflected by the global average of shear stress, $\Sigma$, shown in fig. 5 , where we compare a highly time dependent state (of which instants are shown in the snapshots) with steady flow in the inertial flow regime. The strongly time dependent data confirms the expectation of unsteady flow and reminds on

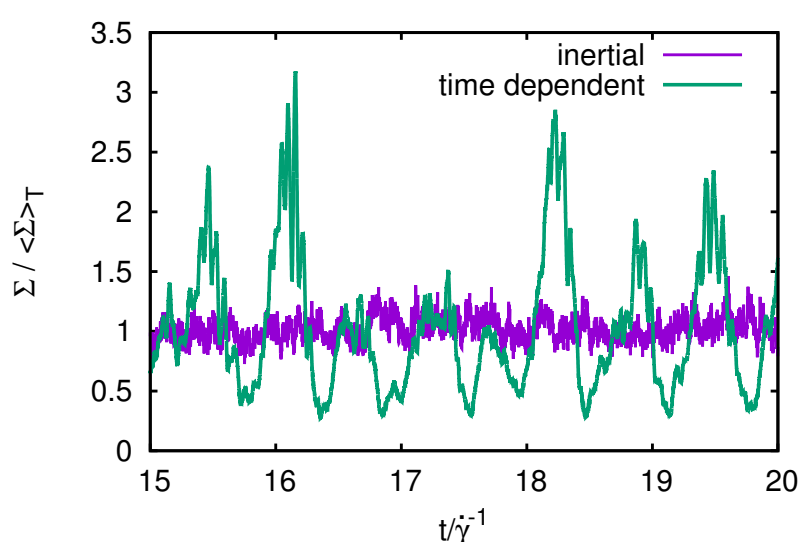

FIG. 5. Shear stress as a function of time leading to time dependent flow with large amplitude (green, $\dot{\gamma}=1.25 \times 10^{-4}$ ). For comparison, we show a time series in the inertial flow regime (purple, $\dot{\gamma}=1 \times 10^{-5}$ ). $N=80000, \varphi=0.7975$.

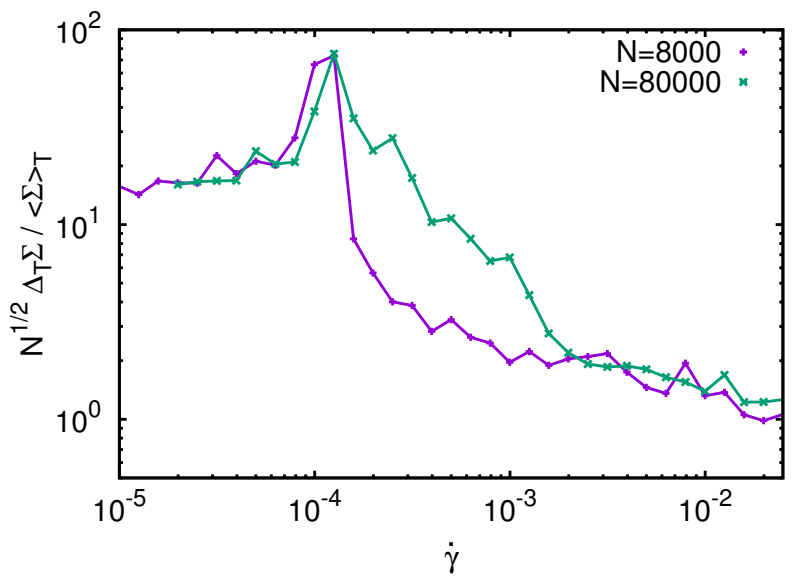

FIG. 6. Relative standard deviation of the measured global stress normalized by $1 / \sqrt{N} \cdot \varphi=0.7975$.

the time series in $[6,26]$. To quantify the degree of the time dependence, we show in fig. 6 the standard deviation of the measured global stress, $\Delta_{T} \Sigma$, for differently sized systems. Asymptotically, for low and large strain rate, the fluctuations scale with the square root of the number of particles, $\sqrt{N}$. In the large system fluctuations show a broad peak $\left(\dot{\gamma}=10^{-4}-10^{-3}\right)$ as an evidence for the time dependence in the shear thickening regime. Over this broad range of strain rate, the large system shows a larger standard deviation than the small system which is yet in the plastic flow regime. The peak in the data of the small systems at $\dot{\gamma} \approx 10^{-4}$ stems from short-lived excursions from an inertial flow state to a plastic flow state (not shown).

In the course of this article, we take averages over heterogeneous and unsteady states. We justify this by the stationarity of the shear stress distribution. As time 


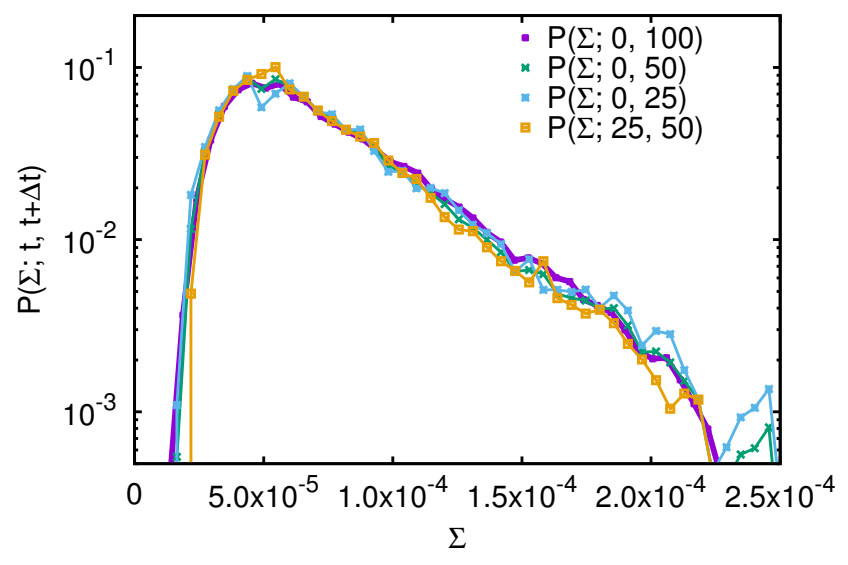

FIG. 7. Distribution of global stress in a specific time window as indicated. Data corresponding to the time dependent time series shown in fig. 5 .

progresses, we measure the global stress, $\Sigma(t)$, and compute the distribution, $P(\Sigma ; t, t+\Delta t)$, between times $t$ and $t+\Delta t$. When this distribution is independent of $t$, the distribution is stationary. In fig. 7 , we show $P(\Sigma ; t, t+\Delta t)$. The distributions coincide. This confirms the stationarity of the stress distribution providing the reason for taking averages over finite time intervals.

The conclusion to this part is that there is only a heterogeneous unsteady state that leads to an intermediate value of stress that gives rise to continuous shear thickening as shown in the flow curve fig. 1. Only averages over unsteady and heterogeneous flow states lead to intermediate stress values which are not accessible by steady flow. However, we showed that characteristic flow features in the dynamics repeat. Moreover, the stress distribution is stationary. The heterogeneity in these states is in stark contrast to the homogeneous states in recent experiments with PMMA particles which also show discontinuous shear thickening due to frictional contacts [6].

\section{B. Stress correlation and anisotropy}

As shown in the previous part, heterogeneous flow states are unsteady but show a stationary stress distribution. We make use of this and time average to extract characteristic lengths and fluctuations by means of stress correlation functions. We contrast our findings to homogeneous and stationary flow.

As seen in the sequence of snapshots in fig. 4, the stress field changes its characteristics in course of its evolution. However, we are interested in the average characterization of the heterogeneous time dependent flow. To quantify the characteristic length and anisotropy we compute stress correlations as in eqn. 5 , with $a_{i}=b_{i}=$ $\sigma_{i}-\langle\sigma\rangle_{N}=\delta \sigma_{i}$. The spatial stress correlation averaged over several snapshots is shown in fig. 8. On short dis-

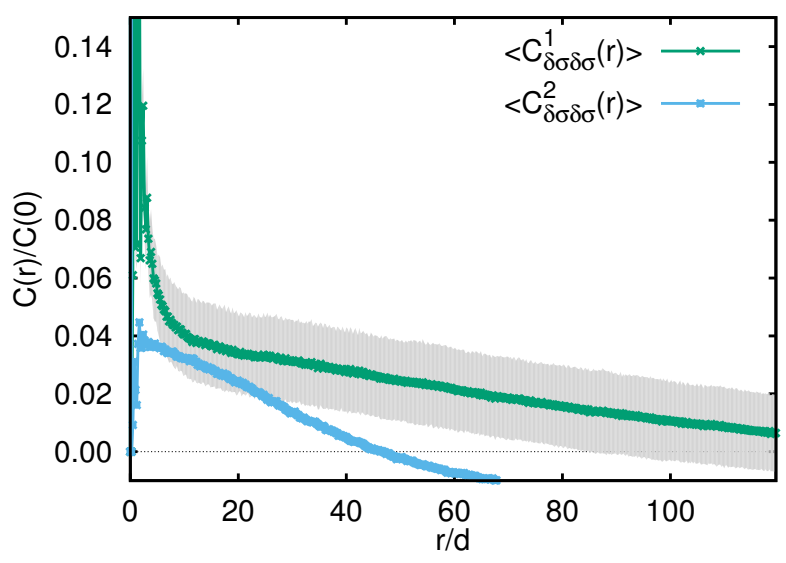

FIG. 8. Stress correlation functions for the heterogeneous time dependent state up to $L / 2 \approx 120$. Average in compression direction, $\left\langle C_{\delta \sigma \delta \sigma}^{1}(r)\right\rangle$, is shown in green and standard deviation is marked in gray. Average stress correlation in dilation direction, $\left\langle C_{\delta \sigma \delta \sigma}^{2}(r)\right\rangle$, is shown in blue. Parameters according to fig. 3 .

tances the stress correlation follows the pair correlation function, $g(r)$ (not shown), which gives rise to a series of maxima and minima for $r<4$. On long distances the correlations decay slowly and exhibit the anisotropy clearly. The average stress correlation in compression direction, $C_{\delta \sigma \delta \sigma}^{1}(r)$, does not decay to zero on a distance $L / 2$. This corresponds with the persistence of stress in compression direction that we see in the snapshot (fig. 3). In dilation direction the correlation function, $C_{\delta \sigma \delta \sigma}^{2}(r)$, crosses zero at about $r \approx 45$ which corresponds roughly to half the width of the stress band shown in the snapshot.

We quantify the decay of the correlation by the length, $l$, where the correlation function, $C_{\delta \sigma \delta \sigma}^{1}(r)$, is decayed to 0.025 [27]. The resulting length is shown as a function of strain rate in fig. 9. We find that the correlation in compression direction behaves non-monotonically as a function of strain rate. In the regime of time dependent flow ( $\dot{\gamma}=10^{-4}-10^{-3}$ and $N \geq 32000$ ), the correlation length peaks. The length ratio, $l / L$, plateaus for large system at intermediate strain rate, see inset of fig. 9. For lower or larger strain rates, the correlation lengths are comparatively small and similar to each other. The small system does not exhibit heterogeneous and time dependent states consistent with the comparatively small correlation length that we measure for $N \leq 8000$. The correlation length in dilation direction (not shown) exhibits the same qualitative behavior but is smaller than in compression direction.

We use an analog of a structure factor of a density field to identify contributions to stress correlations with different wave numbers. For a motivation see appendix C. Bands with low stress and large stress alternate in characteristic large scale structures, see fig. 3. The large scale structures are associated with contributions to small wave numbers in the analog of the structure fac- 


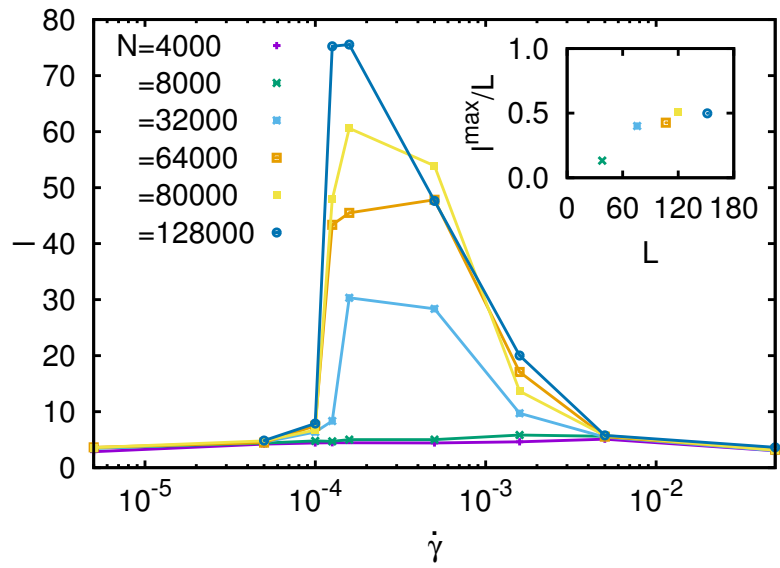

FIG. 9. Main: Distance, $l$, where the correlation function, $C_{\delta \sigma \delta \sigma}^{1}(r)$, is decayed, as a function of strain rate. Inset: Relative length, $l^{\max } / L$, for $\dot{\gamma}=1.58 \times 10^{-4}$ as a function of linear system size, $L$.

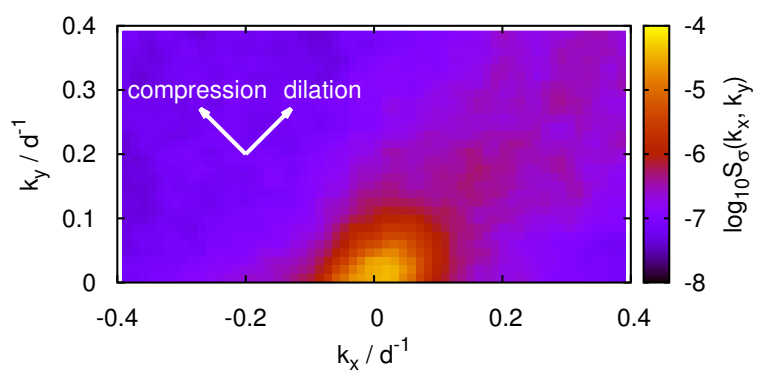

FIG. 10. The Structure factor for heterogeneous time dependent flow exhibits strong anisotropy and shows a large contribution in dilation direction for small wave numbers. Arrows indicate compression and dilation direction. Parameters according to fig. 3 .

tor for the stress field:

$$
N S_{\sigma}(\mathbf{k})=\langle\hat{\sigma}(\mathbf{k}) \hat{\sigma}(-\mathbf{k})\rangle_{T}-\langle\hat{\sigma}(\mathbf{k})\rangle_{T}\langle\hat{\sigma}(-\mathbf{k})\rangle_{T} .
$$

Figure 10 shows the structure factor of the stress field, eq. 8, for time dependent flow. A large contribution (bright) at low wave numbers in dilation direction signals the heterogeneity that corresponds to the large scale structure in the snapshot. Only small contributions are found in directions other than dilation direction or for larger wave numbers. Only the heterogeneous time dependent regime shows a remarkable peak for the smallest wave numbers which can be resolved. This wave number corresponds to the size of the stress band that we see in the snapshot (fig. 3) and suggests that the system requires a certain size to build up heterogeneous and time dependent states. The inertial flow regime for low strain rate and the plastic flow regime for large strain rate do not show long ranged structures (not shown), i.e., the system is homogeneous.

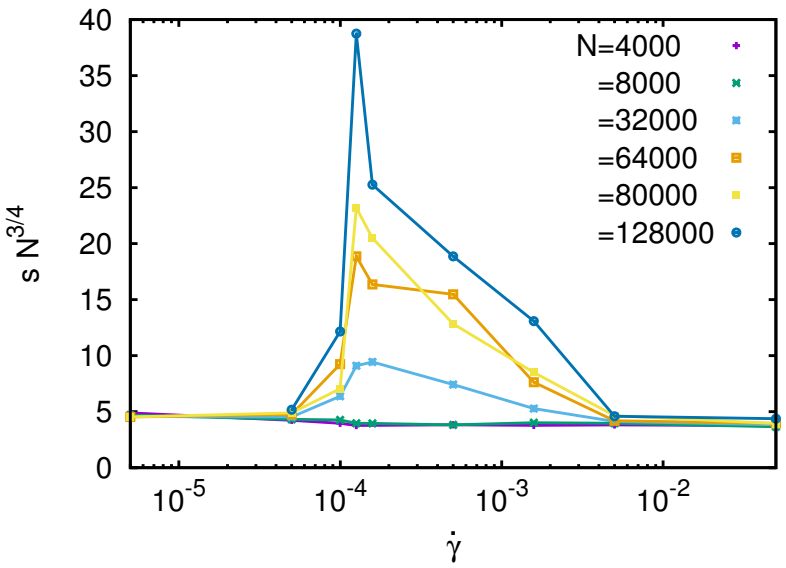

FIG. 11. Average standard deviation of the stress correlation in compression direction, $s$, as a function of strain rate. The $N$-dependence is explained in appendix B.

Now, we want to examine the fluctuations of the stress correlation functions. As shown in fig. 8 (gray area), we compute the binwise standard deviations of the stress correlation to characterize fluctuations in a distance $r / L$. To compare different systems by means of a single number, we average the standard deviations in compression direction over all $N_{b}$ bins:

$$
s=\frac{1}{N_{b}} \sum_{i}^{N_{b}} \operatorname{std} C_{\delta \sigma \delta \sigma}^{1}\left(r_{i}\right) .
$$

Figure 11 shows $s$ as a function of strain rate. The average standard deviation confirms that the small system does not show time dependent behavior while the large system shows highly unsteady dynamics in the continuous shear thickening regime.

In this part, we examined average properties of correlations and the structure of the stress field. Even though the flow is unsteady, we can distinguish clearly between compression and dilation direction, and, more importantly, we report a characteristic length, which is given by the average decay of the stress correlation function. Moreover, the fluctuations of the correlation functions reflect the dynamics of the heterogeneous unsteady flow.

\section{Apparent shear thickening and shear jamming}

In this section we link results with controlled strain rate and results with controlled stress together. In small systems, this relates discontinuous shear thickening to shear jamming. In large systems we find continuous shear thickening and time dependent states instead.

First, we focus on strain rate controlled simulations. The stress that is shown in the flow curve, fig. 1 , is a result of time-averaging over possibly metastable or time dependent states. Examples of such states are shown 


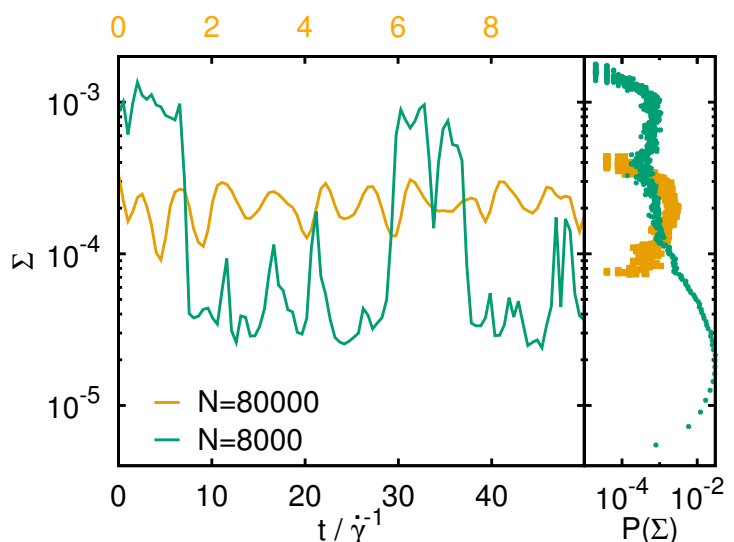

FIG. 12. Left: Smoothed time series, $\Sigma(t)$, for $\varphi=0.7975$. Small system $(N=8000): \dot{\gamma}=1.125 \times 10^{-4}$ leads to temporal coexistence of metastable states at low and large stress. Large system $(N=80000): \dot{\gamma}=1.58 \times 10^{-4}$ leads to intermediate but unsteady stress (time at top axis). Right: Corresponding stress distributions.

in the time series in fig. 12. In green we show a time series of the small system with an intermediate strain rate where flow is metastable: Both, large and low stress states, are attained alternately. The shear stress jumps between the two states which differ in stress by more than an order of magnitude. This jump determines the discontinuous shear thickening in the flow curve. The orange curve shows a time series of the large system with an intermediate strain rate leading to apparent continuous shear thickening in the flow curve. At this strain rate we see a time dependent state. Remarkably, the time dependent state lives in an interval of stress which is not favored by the small system. Instead, the time series and flow curve of the small system exhibit jumps across that interval of stress. The distribution of the metastable state is bimodal with peaks at metastable values of stress. The peak of the distribution for the time dependent state resides at the local minimum of the distribution of the metastable flow. This reminds on the results in $[15,28]$. Thus there is an unstable band of stress values leading to jumps in the small system and time dependent flow in the large system.

Next, we focus on stress controlled simulations. To probe jamming in the unstable region we impose stress following previous studies [13]. In the unstable regime we observe that the flow ceases and the system jams when the simulation cell is small. Figure 13 shows the time of transient flow in differently sized systems. In small systems, $N=4000-16000$, we find that the system jams after a finite transient time which is characteristic for the stress that is applied. Jamming occurs around the minimum in the bimodal distribution in fig. 12. This is the same range of stress where we find the jumps in the time series fig. 12 or in the flow curve in fig. 1 . In contrast, in large simulation cells, $N>24000$, we cannot

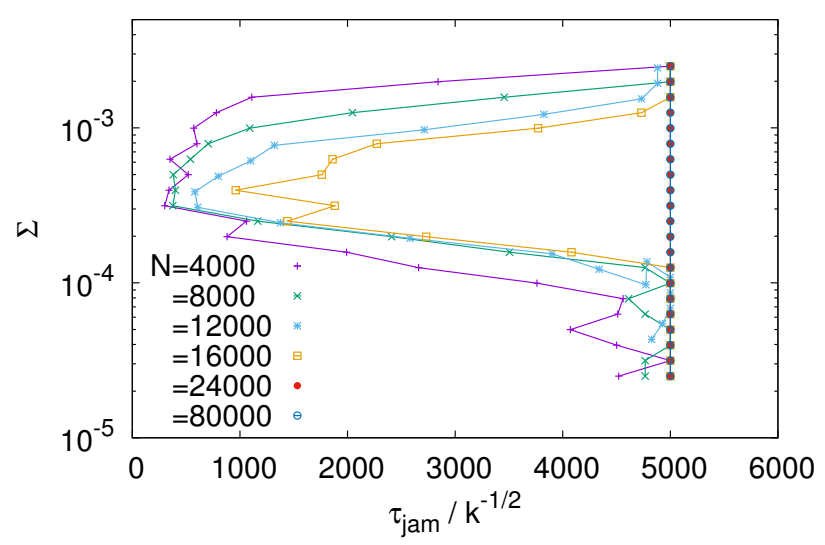

FIG. 13. Time of transient flow, $\tau_{\text {jam }}$, for fixed $\phi=0.7975$ but different system size, $N=4000-80000$. When the system never jams, we show the simulation time (5000).

find shear jamming at this packing fraction but unsteady flow instead.

To conclude this part, the results of simulations with different protocol and differently sized simulation cells directly relate to the flow curves shown in fig. 1 . Small systems follow a sigmoidal flow curve and show metastable and unstable states, i.e., alternating stress at fixed strain rate and shear jamming at fixed shear stress, respectively. When the system is large we observe apparent shear thickening and we cannot find jammed states at this packing fraction in the stress controlled setting. Instead, unstable flow is realized by time dependent states as predicted by the model in [14].

\section{Velocity distributions}

In this section, we investigate the distribution of nonaffine velocities. We describe the time evolution and average characteristics in terms of the width of distribution and relate the findings to the flow curves via the generalized viscosity.

In the heterogeneous state, stress varies by orders of magnitude across the system and these strong gradients suggest a broad distribution of velocity, see fig. 19 for an example of a heterogeneous velocity field. We compute the velocity distributions in gradient direction over the particles, $P\left(v_{y}\right)$, in a single configuration as shown in fig. 14. By inspection of the distributions we find a remarkable non-monotonicity as a function of the strain rate. At large strain rate the width of the velocity distribution is small but increases as the strain rate decreases. In the shear thickening regime at intermediate strain rate the distribution is the widest. At low strain rate the distribution is again narrow. For all strain rates the distribution is peaked at zero and symmetric due to the problem's symmetry. Hence, the width of the distribution is 


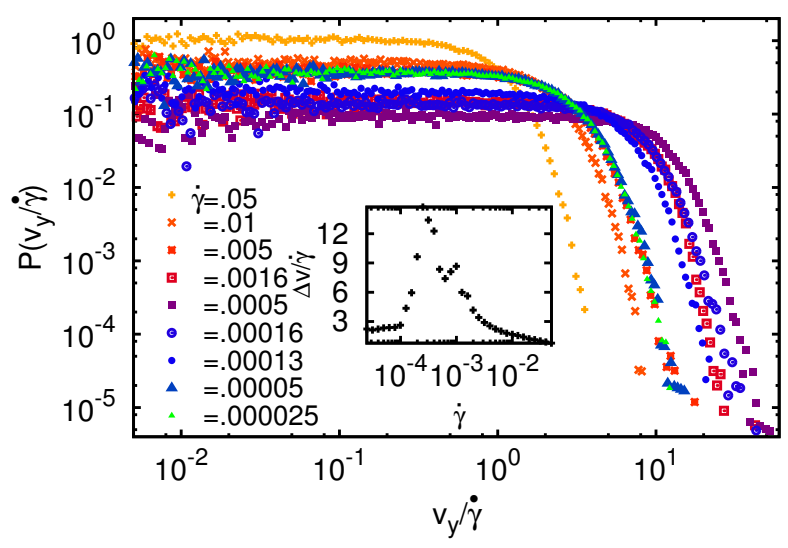

FIG. 14. Velocity distribution of individual snapshots at different strain rates for $\phi=0.7975$ and $N=80000$. Inset: Width of the velocity distribution as a function of strain rate.

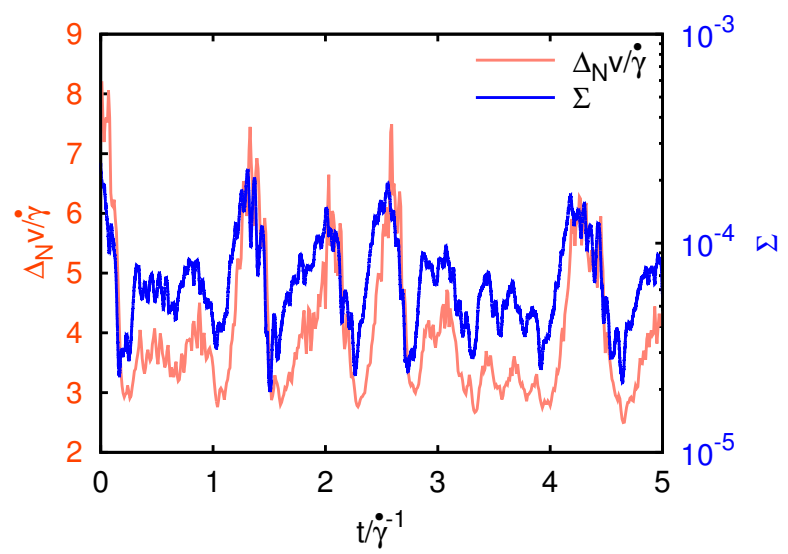

FIG. 15. Time series for the width of the velocity distribution (left axis, red) and the shear stress (right axis, blue). Parameters according to fig. 3 .

a suitable measure for the typical velocity of a particle in the system. These widths are shown in the inset of fig. 14, confirming the non-monotonic behavior.

However, the velocity distributions are affected by the unsteady character of heterogeneous flow. At each instant of a simulation, $t$, we compute $P\left(v_{y}, t\right)$ individually and extract the width of the velocity distribution as a function of time. These widths, $\Delta_{N} v_{y}(t)$, are shown as a time series in fig. 15. The time series of the standard deviation reflect the overall time dependence of the system as could be observed in other quantities, e.g., the shear stress which is shown for comparison. We characterize the widths of the velocity distribution by fig. 16, where the corresponding time averaged standard deviations, $\left\langle\Delta_{N} v_{y}(t)\right\rangle_{T}$, are shown. This quantity shows the same behavior as the flow curve by means of the generalized viscosity, $\eta=\sigma / \dot{\gamma}^{2}$ (fig. 17): a discontinuity in

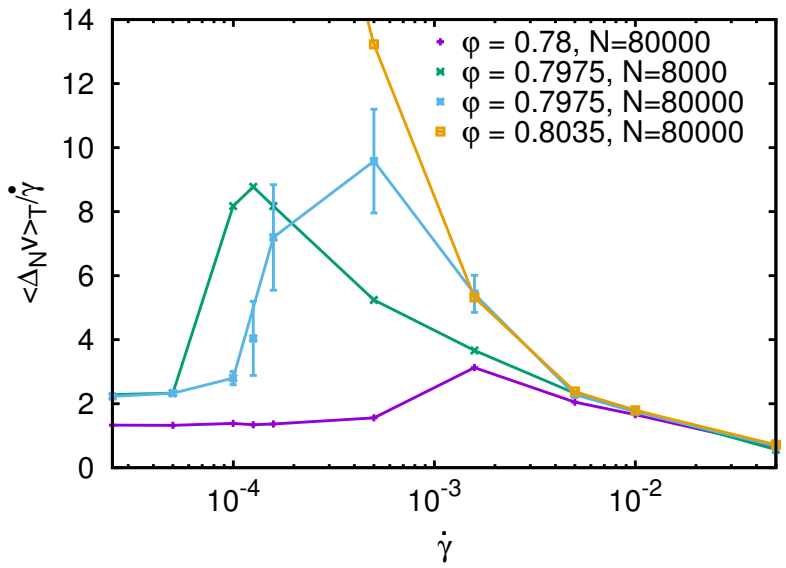

FIG. 16. Time averaged width of the velocity distributions showing non-monotonic behavior.

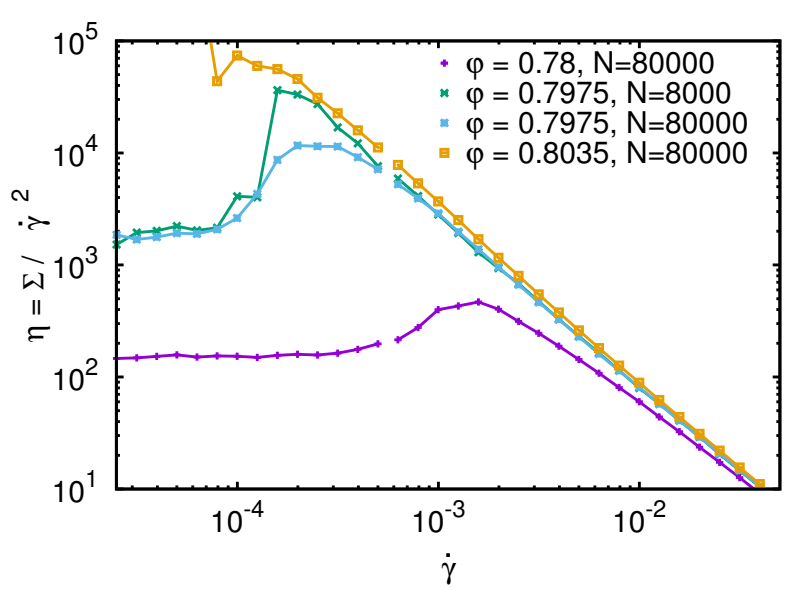

FIG. 17. Generalized viscosity showing non-monotonic behavior.

small systems and continuity as a result of taking the average over time dependent quantities in the large system for $\phi=0.7975$. The standard deviation has a maximum near the jump in the small system and in the time dependent regime in the large system. A packing fraction of $\phi=0.78$ leads to only a small increase in viscosity and in the width of the velocity distribution. The densest system with $\phi=0.8035$ does not settle in the inertial flow branch and the viscosity and the width of the velocity distribution grow as the strain rate decreases.

In this section, we showed that velocity fluctuations are linked to the effective viscosity of the system as also observed in simulations of particles suspended in a viscous fluid [28]. Both, the width of the velocity distribution and the generalized viscosity, shown in fig. 17 , show the same qualitative dependence on the flow state. 


\section{DISCUSSION AND CONCLUSION}

We studied the shear thickening phenomenon of dry frictional granular particles. Large simulation cells exhibit continuous shear thickening across an unstable interval of stress. Continuous shear thickening is accompanied by heterogeneous and unsteady flow. The heterogeneous flow states, which were first reported in [14], possess a strong anisotropy and a characteristic length, which is considerably larger than the particle size. The characteristic length grows linearly with the system size. A length, which is based on vortexlike nonaffine displacements, linearly dependent on system size is reported in [29]. This is in accordance with large-scale structures that we observe by visual inspection in the velocity field, i.e., vortexlike structures and additional almost parallel moving particles (red), see fig. 19.

In small simulation cells, the application of stress in the unstable interval leads to shear jamming, see also [13]. Strain rate controlled simulations show jumps across the unstable interval, see also $[13,15]$, giving rise to discontinuous shear thickening. The system size is found to be a relevant property for the shear thickening scenario. This is in stark contrast to the claim that hysteresis (accompanied by discontinuous shear thickening) persists in the thermodynamic limit [12], where we expect continuous shear thickening instead.

The investigation of the nonaffine velocity distributions shows that their width is a sensible proxy for the state of the system: The time dependence of stress and width of the velocity distribution coincide and the average width shows the same strain rate and packing fraction dependencies as the viscosity. A recent study relates velocity fluctuations to dissipation and shows that the major contribution to dissipation stems from a fraction of particles that goes to zero as jamming is approached [30]. We reported a novel phenomenon: a nonmonotonic strain rate dependence of the width of velocity distributions below jamming, i.e., the fastest particles are found in the continuous shear thickening regime. It remains elusive how these particles contribute to the dissipation, and thereby to the rheology and the unsteady nature of flow.

\section{ACKNOWLEDGMENTS}

We gratefully acknowledge financial support by the Deutsche Forschungsgemeinschaft via programs FOR 1394 and Emmy Noether He 6322/1-1. MG thanks Ehsan Irani and Knut Heidemann for fruitful discussions.

\section{Appendix A: Local coordination number and nonaffine velocity}

The features that we see in the stress can be measured in other quantities, too, e.g., the local coordination num-

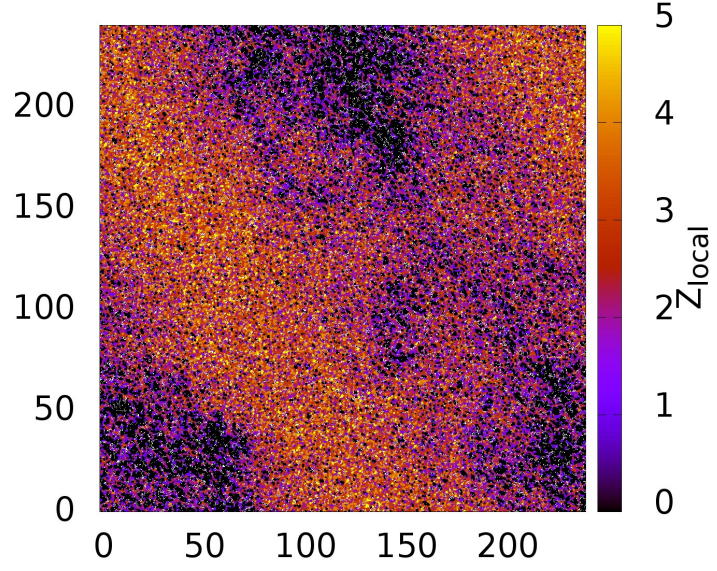

FIG. 18. Snapshot of the local coordination number, $Z_{\text {local }}$, of the configuration in fig. 3 .

ber and the nonaffine velocities. This correspondence is shown in figs. 18 and 19, where we show the local coordination number, $Z_{\text {local }}$, and the nonaffine velocity, $\mathbf{v}_{\text {na }}$, respectively. In the region of large stress, the coordination number is large and in the regions of low stress the coordination number is low. Strikingly, the state, which is shown, is unsteady: There is a large region of particles (red) that moves to the bottom left with a velocity that is considerably larger than the strain rate. Regions of large nonaffine velocity correspond to regions of large stress and a low nonaffine velocity corresponds to low stress. An strong correlation between mean coordination number and average stress was already reported [21, 31]. Here we want to emphasize the local relation.

\section{Appendix B: Scaling of the average standard deviation of the correlation functions}

Here, we demonstrate the dependence $s(N) \propto N^{-3 / 4}$. We consider average correlation functions with $N_{b}$ bins stemming from $N_{T}$ samples with $N$ particles each. Due to the computational complexity and data storage, we have chosen: $N \propto 1 / N_{T}$. Moreover, the number of bins is constant, $N_{b}=$ const. $N_{T}$ snapshots contribute to the binwise standard deviation, $\operatorname{std}\left(C\left(r_{i}\right)\right)$ with bin $i$. The number of pairs of particles per snapshot that fall in a bin is called $N_{d}$. Therefore, we expect scaling as:

$$
\operatorname{std}\left(C\left(r_{i}\right)\right) \propto 1 / \sqrt{N_{T} N_{d}}
$$

$N_{d}$ is proportional to the size of the bins times the number of pairs of particles, i.e., $N_{d} \propto L / N_{b} N^{2} \propto N^{5 / 2} / N_{b}$. 


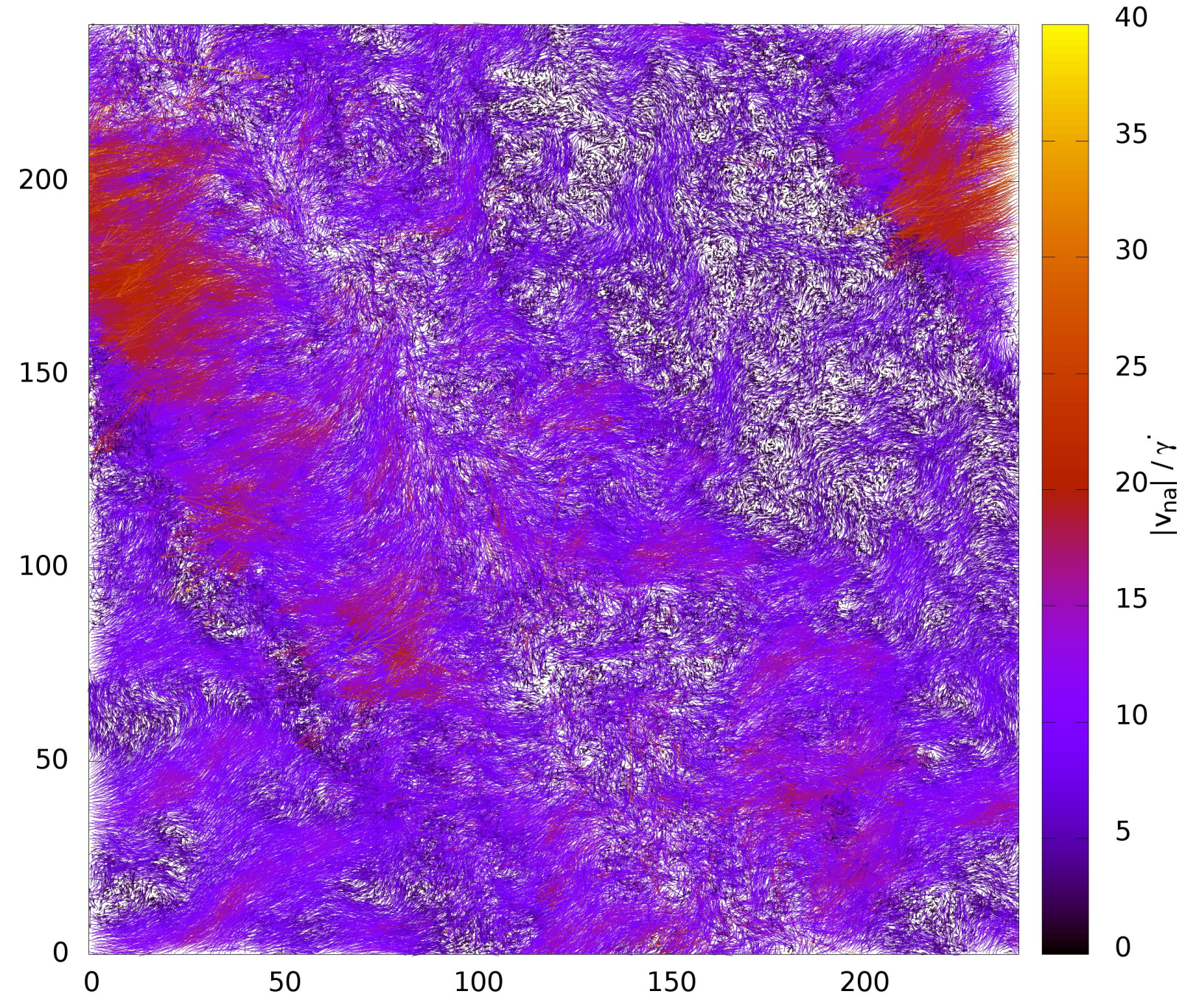

FIG. 19. Snapshot of the local nonaffine velocity, $\mathbf{v}_{\mathbf{n a}}$, of the configuration in fig. 3. Nonaffine velocities are indicated by arrows. The length and color scale with the absolute value of the nonaffine velocity. The reader of a digital copy is invited to zoom in and investigate the rich structure.

In total:

$$
\operatorname{std}\left(C\left(r_{i}\right)\right) \propto N^{1 / 2} / N^{5 / 4}=N^{-3 / 4} .
$$

For all system sizes, the number of bins is constant and averaging does not affect the scaling and therefore $s(N) \propto N^{-3 / 4}$.

\section{Appendix C: Definition of the analog of the} structure factor for the stress field 
constitute a field localized at the particles' positions:

$$
\sigma(r ; t)=\sum_{i=1}^{N} \sigma_{i}(t) \delta\left(r-r_{i}(t)\right)
$$

The field, $\sigma(r ; t)$, has a spatial average, $\langle\sigma(r ; t)\rangle_{N}$, and $\delta \sigma(r ; t)=\sigma(r ; t)-\langle\sigma(r ; t)\rangle_{N}$ is the deviation from the spatial average. Correlations in space are measure by:

$$
\begin{aligned}
C\left(r, r+r^{\prime} ; t\right) & =\delta \sigma(r ; t) \delta \sigma\left(r+r^{\prime} ; t\right) \\
& =\sigma(r ; t) \sigma\left(r+r^{\prime} ; t\right)-\langle\sigma(r ; t)\rangle_{N}^{2} .
\end{aligned}
$$

The assumption that correlations are only dependent on the displacement between two points yields:

$$
C(r ; t)=\delta \sigma(0 ; t) \delta \sigma(r ; t)
$$

With this assumption, the Fourier transform only depends on the wave vector, $k$, and time:

$$
\hat{C}(k ; t)=\sum_{i, j} \delta \sigma_{i}(t) \delta \sigma_{j}(t) \exp \left[-i\left(k\left(r_{i}(t)-r_{j}(t)\right)\right)\right] .
$$

With the transformed field

$$
\hat{\delta \sigma}(k ; t)=\sum_{i} \delta \sigma_{i}(t) \exp \left(-i k r_{i}(t)\right)
$$

we can write:

$$
\hat{C}(k ; t)=\hat{\delta \sigma}(k ; t) \hat{\delta \sigma}(-k ; t) .
$$

The structure factor, $S_{\sigma}(k ; t)$, of a field, $\sigma(r ; t)$, is defined as the fluctuating Fourier components averaged over an ensemble. Here, the ensemble that we consider is given by the time evolution of our dynamics. Thus we have with $N$ particles:

$$
\begin{aligned}
N S_{\sigma}(k) & =\langle\hat{\delta \sigma}(k ; t) \hat{\delta \sigma}(-k ; t)\rangle_{T} \\
& =\left\langle|\hat{\sigma}(k ; t)|^{2}\right\rangle_{T}-\left|\langle\hat{\sigma}(k ; t)\rangle_{T}\right|^{2} .
\end{aligned}
$$

[1] D. Bi, S. Henkes, K. E. Daniels, and B. Chakraborty. Annual Review of Condensed Matter Physics 6, 63 (2015).

[2] N. V. Brilliantov and T. Pöschel. Kinetic theory of granular gases. Oxford University Press (2010). ISBN 9780198530381.

[3] A. J. Liu and S. R. Nagel. Nature 396, 21 (1998).

[4] A. J. Liu and S. R. Nagel. Annual Review of Condensed Matter Physics 1, 347 (2010).

[5] B. M. Guy, M. Hermes, and W. C. K. Poon. Physical Review Letters 115, 088304 (2015).

[6] Z. Pan, H. de Cagny, B. Weber, and D. Bonn. Physical Review E 92, 032202 (2015).

[7] J. R. Royer, D. L. Blair, and S. D. Hudson. Physical Review Letters 116, 188301 (2016).

[8] D. Bi, J. Zhang, B. Chakraborty, and R. P. Behringer. Nature 480, 355 (2011).

[9] A. Fall, F. Bertrand, D. Hautemayou, C. Mezière, P. Moucheront, A. Lemaître, and G. Ovarlez. Physical Review Letters 114, 098301 (2015).

[10] W. Jiang, S. Xuan, and X. Gong. Applied Physics Letters 106, $151902(2015)$.

[11] M. P. Ciamarra, R. Pastore, M. Nicodemi, and A. Coniglio. Physical Review E 84, 041308 (2011).

[12] M. Otsuki and H. Hayakawa. Physical Review E 83, 051301 (2011).

[13] M. Grob, C. Heussinger, and A. Zippelius. Physical Review E 89, 050201 (2014).

[14] M. Grob, A. Zippelius, and C. Heussinger. Physical Review E 93, 030901 (2016).

[15] R. Mari, R. Seto, J. F. Morris, and M. M. Denn. Journal of Rheology page 32 (2014).

[16] M. Hermes, B. M. Guy, G. Poy, M. E. Cates, M. Wyart, and W. C. K. Poon. arXiv (2015)
[17] M. Wyart and M. E. Cates. Physical Review Letters 112, 098302 (2014).

[18] N. Fernandez, R. Mani, D. Rinaldi, D. Kadau, M. Mosquet, H. Lombois-Burger, J. Cayer-Barrioz, H. J. Herrmann, N. D. Spencer, and L. Isa. Physical Review Letters 111, 108301 (2013).

[19] P. D. Olmsted. Rheologica Acta 47, 283 (2008).

[20] C. S. Campbell. Journal of Fluid Mechanics 465, 261 (2002).

[21] S. Chialvo, J. Sun, and S. Sundaresan. Physical Review E 85, 021305 (2012).

[22] P. A. Cundall and O. D. L. Strack. A discrete numerical model for granular assemblies (1979).

[23] L. E. Silbert, D. Erta, G. S. Grest, T. C. Halsey, D. Levine, and S. J. Plimpton. Physical Review E 64, $051302(2001)$.

[24] E. DeGiuli, J. N. McElwaine, and M. Wyart. arXiv (2015).

[25] S. Henkes, D. A. Quint, Y. Fily, and J. M. Schwarz. Physical Review Letters 116, 028301 (2016).

[26] D. Lootens, H. Van Damme, and P. Hébraud. Physical Review Letters 90, 178301 (2003).

[27] We have checked that the resulting picture does not depend on this threshold within a reasonable interval.

[28] C. Heussinger. Physical Review E 88, 050201 (2013).

[29] C. E. Maloney. Physical Review Letters 97, 1 (2006)

[30] P. Olsson. Physical Review E 93, 042614 (2016).

[31] E. Aharonov and D. Sparks. Physical Review E 60, 6890 (1999). 



\section{Active microrheology of driven granular particles}

Reprinted article with permission from

Wang, Ting and Grob, Matthias and Zippelius, Annette and Sperl, Matthias

Physical Review E 89042209 (2014)

http://dx.doi.org/10.1103/PhysRevE.89.042209

Copyright (2014) by the American Physical Society. 



\title{
Active microrheology of driven granular particles
}

\author{
Ting Wang, ${ }^{1}$ Matthias Grob, ${ }^{2}$ Annette Zippelius, ${ }^{2,3}$ and Matthias Sperl ${ }^{1}$ \\ ${ }^{1}$ Institut für Materialphysik im Weltraum, Deutsches Zentrum für Luft und Raumfahrt (DLR), 51170 Köln, Germany \\ ${ }^{2}$ Georg-August-Universität Göttingen, Institut für Theoretische Physik, Friedrich-Hund-Platz, 1, 37077 Göttingen, Germany \\ ${ }^{3}$ Max-Planck-Institut für Dynamik und Selbstorganisation, Am Faßberg 17, 37077 Göttingen, Germany
}

(Received 23 December 2013; published 28 April 2014)

\begin{abstract}
When pulling a particle in a driven granular fluid with constant force $F_{\text {ex }}$, the probe particle approaches a steady-state average velocity $v$. This velocity and the corresponding friction coefficient of the probe $\zeta=F_{\text {ex }} / v$ are obtained within a schematic model of mode-coupling theory and compared to results from event-driven simulations. For small and moderate drag forces, the model describes the simulation results successfully for both the linear as well as the nonlinear region: The linear response regime (constant friction) for small drag forces is followed by shear thinning (decreasing friction) for moderate forces. For large forces, the model demonstrates a subsequent increasing friction in qualitative agreement with the data. The square-root increase of the friction with force found in [Fiege et al., Granul. Matter 14, 247 (2012)] is explained by a simple kinetic theory.
\end{abstract}

DOI: 10.1103/PhysRevE.89.042209

PACS number(s): 45.70.-n, 82.70.Dd, 83.60.Df, 83.10.Gr

\section{INTRODUCTION}

Active microrheology (AM) studies the mechanical response of a many-particle system on the microscopic level by pulling individual particles through the system either with constant force or at constant velocity [1]. While in passive microrheology only the linear response can be probed, AM can also be applied to explore the nonlinear response by imposing large drag forces. An external force $F_{\text {ex }}$ can be imposed by magnetic [2] or optical tweezers [3] to a probe particle embedded in a soft material and then the corresponding steady-state velocity $\langle v\rangle$ is measured by optical microscopy [4]. Recently, AM experiments [2] and simulations [5-7] for dense colloidal suspensions found that (i) in the linear-response region, the friction coefficient of the probe $\zeta=$ $F_{\text {ex }} /\langle v\rangle$ directly indicates the increasing rigidity of the system when approaching the glass transition from the liquid state; (ii) in the nonlinear response region, the friction coefficient tends to decrease to a certain value with increasing pulling force-an effect reminiscent of shear thinning in macrorheology. Both effects could be explained by an extension of mode-coupling theory (MCT) to describe AM [8,9].

Within the MCT interpretation, the description of AM for colloidal suspensions is based on the existence of a glass transition in such systems. The interplay between growing density correlations by glass formation and the suppression of those correlations by microscopic shear explains the observed behavior of the friction microscopically. In addition to colloidal suspensions, a glass transition is also predicted by MCT for driven granular systems [10-12]. Here, the energy lost in the dissipative interparticle collisions is balanced by random agitation. Starting from the nonequilibrium steady state of this homogeneously driven granular system, the corresponding AM shall be elaborated below.

In AM of granular matter similar phenomena as in colloidal suspensions are found: (i) Dramatic increasing of the friction coefficient and (ii) shear thinning have been identified in experiments with horizontally vibrated granular particles $[13,14]$, and both effects were reproduced in recent simulations of a two-dimensional granular system [15]. Moreover, for large pulling forces in the simulation, beyond the thinning regime the friction coefficients increase again and exhibit power-law behavior close to a square root: $\zeta\left(F_{\mathrm{ex}}\right) \propto \sqrt{F_{\mathrm{ex}}}$ for $F_{\text {ex }} \gg 1$. This finding is in contrast to the predicted constant friction (second linear regime) in the colloidal hard-sphere system [8,9]. In the following, we shall demonstrate how a schematic MCT model can capture the increase with friction for large forces. In addition, for dilute systems we shall derive a square-root law for large forces exactly.

\section{DYNAMICS OF A GRANULAR INTRUDER}

The driven granular system under consideration is composed of $N$ identical particles interacting with each other. One probe particle experiences a constant pulling force $\boldsymbol{F}_{\mathrm{ex}}$. The dynamics of the system is given for every particle $i$ by the equation of motion

$$
m \dot{\boldsymbol{v}}_{i}=-\zeta_{0} \boldsymbol{v}_{i}+\boldsymbol{f}_{\text {int }}^{i}+\boldsymbol{\eta}_{i}+\boldsymbol{F}_{\mathrm{ex}} \delta_{i, s},
$$

where $\zeta_{0}$ is the bare friction depending on the friction of the surrounding medium, which for large values is reminiscent of a colloidal suspension. The force $f_{\text {int }}^{i}$ is the particle interaction force, which for the granular case is typically given by a collision rule to include the energy loss at contact. $\boldsymbol{\eta}_{i}$ is a random driving force satisfying a fluctuation dissipation relation $\left\langle\boldsymbol{\eta}_{i}(t) \boldsymbol{\eta}_{j}\left(t^{\prime}\right)\right\rangle=2 \zeta_{0} k_{B} T / m \delta_{i, j} \delta\left(t-t^{\prime}\right)$, and the constant pulling force $\boldsymbol{F}_{\text {ex }}$ is imposed on the probe particle (denoted $s$ ) only. Models similar to Eq. (1) have been proposed frequently and elaborated on regarding their dynamics in the description of driven granular systems [16].

\section{A. Schematic model}

The use of MCT for the description of glassy dynamics can be found fully self-contained and in considerable detail in recent reviews and books such as Ref. [18]. We shall outline briefly the general framework of the theory as follows. MCT describes the dynamics in the liquid state by correlation functions for which one can derive the microscopic equations of motion for the system under consideration. Using projection-operator formalisms, the equations of motion can be reformulated exactly as integrodifferential equations for 
the correlation functions where the integral terms represent memory effects. To close the equations, an approximationthe mode-coupling factorization-is invoked, and the resulting equations are solved numerically by asymptotic expansion. In addition to the full microscopic MCT equations, simplified so-called schematic models can be used that capture the mathematical structure of the full theory but are easier to solve [18]. The MCT formalism has been extended to dissipative granular dynamics in Ref. [12]. The theory predicts a glass transition for a driven granular fluid for all degrees of inelasticity, parametrized by the coefficient of resitution $\varepsilon$. Hence, there is a glass transition line in the phase diagram spanned by volume fraction and $\varepsilon$. In the following, we build on these results to discuss the dynamics of a single intruder pulled through a dissipative granular medium. As a first step toward a full MCT theory, we generalize schematic models that have been devised for the dynamics of an intruder in a colloidal fluid [9] to the case of an intruder inside a dissipative granular host fluid.

The friction coefficient of the probe can be calculated by the integration-through-transients (ITT) method combined with the MCT approximation. This procedure was first applied to describe the macrorheology [17] and was later extended to AM for colloidal suspension [8,9]. We follow the approach in Refs. [8,9] to construct a schematic MCT model for driven granular systems. Different from colloidal systems, the equations of motion for the density autocorrelation functions for both the bulk system and the probe particle, $\phi_{q}(t):=$ $\left\langle\rho_{\boldsymbol{q}}(t) \rho_{\boldsymbol{q}}^{*}\right\rangle /\left\langle\rho_{\boldsymbol{q}} \rho_{\boldsymbol{q}}^{*}\right\rangle$ and $\phi_{q}^{s}(t):=\left\langle\exp \left\{i \boldsymbol{q} \cdot\left[\boldsymbol{r}_{s}(t)-\boldsymbol{r}_{s}\right]\right\}\right\rangle$, respectively, include a second time derivative, because granular systems are not overdamped. Here, $\langle\cdots\rangle$ denotes the ensemble average and $\rho_{\boldsymbol{q}}(t):=\sum_{i=1}^{N} e^{i \boldsymbol{q} \cdot \boldsymbol{r}(t)}$ is the Fourier transform of the density. In schematic models the dependence of the correlation functions on the wave vector $\boldsymbol{q}$ is ignored. Instead, the correlation functions are evaluated at one particular wavenumber $q_{0}$, e.g., the maximum of the structure factor. The equations for $\phi(t)=\phi_{q_{0}}(t)$ and $\phi_{s}(t)=\phi_{q_{0}}^{s}(t) \mathrm{read}$

$$
\begin{aligned}
\ddot{\phi}(t)+v \dot{\phi}(t)+\Omega^{2}\left[\phi(t)+\int_{0}^{t} d \tau m(t-\tau) \dot{\phi}(\tau)\right] & =0 \\
\ddot{\phi}_{s}(t)+v_{s} \dot{\phi}_{s}(t)+\Omega_{s}^{2}\left[\phi_{s}(t)+\int_{0}^{t} d \tau m_{s}(t-\tau) \dot{\phi}_{s}(\tau)\right] & =0 .
\end{aligned}
$$

The first 3 terms in Eq. (2) describe oscillations with frequency $\Omega\left(\Omega_{s}\right)$, which are damped with rate $v\left(v_{s}\right)$. The last terms account for memory effects. In a schematic MCT model (called $F_{12}$ model) the memory kernels are approximated by nonlinear functions of the correlation functions as follows:

$$
\begin{aligned}
m(t) & =v_{1} \phi(t)+v_{2} \phi^{2}(t) \\
m_{s}(t) & =v_{A} \phi(t) \operatorname{Re}\left[\phi_{s}(t)\right] .
\end{aligned}
$$

The host fluid is assumed to be large enough so that its density correlation function is not affected by the external pulling force $F_{\text {ex }}$. Consequently, the collective dynamics is completely decoupled in Eqs. (2) and (3). Here, we are interested in the long-time behavior of $\phi(t)$, which is not affected by $\nu$ and $\Omega$, so that we take $\nu=\Omega=1$ for simplicity. The control parameters $\left(v_{1}, v_{2}\right)$ of the host fluid determine the liquid or glassy state together with the distance of a chosen state point from a glass-transition line, which in the case of schematic models can be calculated analytically [18]. The state points $\left(v_{1}, v_{2}\right)=\left[v_{1}^{c}(1+\sigma), v_{2}^{c}(1+\sigma)\right]$ are specified by a distance $\sigma$ to the transition line given by $v_{1}^{c}=v_{2}^{c}\left(2 / \sqrt{v}_{2}^{c}-1\right)$ with the specific choice $v_{2}^{c}=2$ for the transition point.

The coupling strength between the probe and the host system is indicated by $v_{A} . \Omega_{s}^{2}$ is the effective frequency of the correlation function of the probe and set to be $\Omega_{s}^{2}=1-i F_{\mathrm{ex}}$. This result can be obtained exactly from Eq. (1) by considering the limit of vanishing interacting force:

$$
\begin{aligned}
\phi_{\boldsymbol{q}}^{s}(t) & =\exp \left(-\Omega_{s, \boldsymbol{q}}^{2} t^{2}\right) \\
\Omega_{s, \boldsymbol{q}}^{2} & =\frac{\boldsymbol{q}}{m} \cdot\left(\boldsymbol{q} k_{B} T-i \boldsymbol{F}_{\mathrm{ex}}\right) \rightarrow \frac{q_{0}^{2}}{m} k_{B} T\left(1-i \frac{F_{\mathrm{ex}}}{q_{0} k_{B} T}\right) .
\end{aligned}
$$

$v_{s}$ describes the dynamics of the density correlator of the probe for short time scales. In order to assure that $\left|\phi_{s}(t)\right| \leqslant 1$, it is required that

$$
v_{s}>F_{\mathrm{ex}},
$$

which is obtained by solving the second equation in Eqs. (2) without the memory kernel. By integration of the density autocorrelators [8,9], we get the expression for the effective friction of the probe as

$$
\zeta / \zeta_{0}=1+\frac{q_{0}^{2}}{\zeta_{0}} k_{B} T \int_{0}^{\infty} d t \operatorname{Re}\left[\phi_{s}(t)\right] \phi(t) .
$$

Equation (5) shows clearly that $v_{s}$ depends on the pulling force. However, its dependence beyond the inequality can only be obtained from a microscopic theory. Within the schematic model, we have investigated several functional forms of $v_{s}\left(F_{\text {ex }}\right)$ complying with the constraint in Eq. (5). The simplest choice is the straightforward generalization of the colloidal case: (a) $v_{s}=1+F_{\mathrm{ex}}$. As shown below, this choice is not compatible with the data from simulations. This has led us to consider case (b) $v_{s}=1+F_{\mathrm{ex}}^{2}$. Ultimately, a microscopic derivation needs to show if case (b) can be obtained from the microscopic equations of motion, cf. [9].

The respective numerical solutions for the force-dependent friction coefficients are given in Fig. 1, where $\sigma$ indicates the distance from the glass transition. The force-dependent friction of the probe exhibits three characteristic regimes. For small pulling forces in both models, the friction coefficient is constant, or equivalently, the average velocity of the probe is proportional to the external pulling force. This region extends to external forces of order unity and describes a linear response. When approaching the glass transition, the friction increases drastically as the correlation functions in Eq. (6) extend to increasingly longer time scales. Starting around $F_{\text {ex }} \approx 1$, the linear-response regime ends and gives rise to shear thinning: The friction decreases and it is proportionally easier to pull the particle. Equivalently, the average velocity of the intruder increases faster than linear with external force. For the model in the left panel of Fig. 1, the friction $\zeta$ approaches the limiting value given by the bare friction $\zeta_{0}$ and remains there for yet higher forces. This model hence describes behavior similar to the colloidal results for Newtonian microscopic dynamics. For the model in the right panel of Fig. 1, the friction approaches a minimum around $F_{\mathrm{ex}} \approx 100$ and starts increasing for higher pulling forces. 


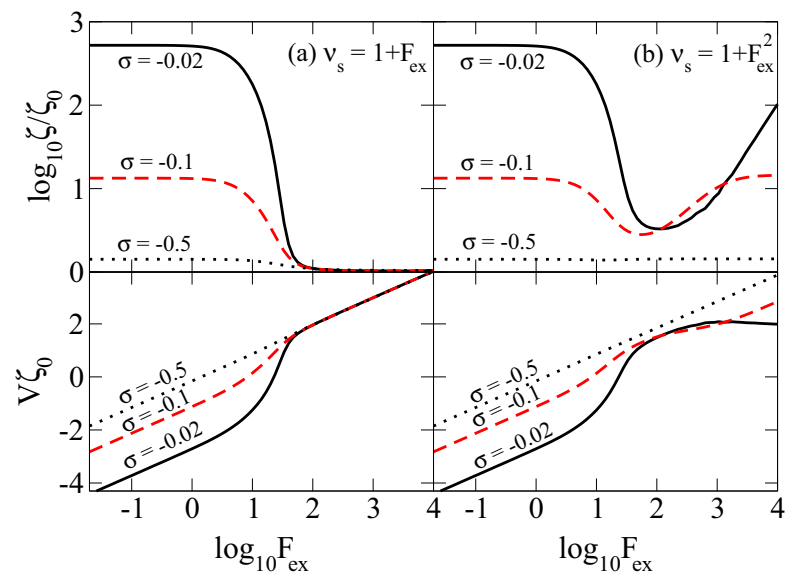

FIG. 1. (Color online) Force-dependent friction coefficient (upper panels) for different models of the damping $v_{s}\left(F_{\mathrm{ex}}\right)$ together with the steady-state velocities (lower panels). $\sigma$ specifies the distance from the glass-transition point in the schematic model.

In comparison, the models in Figs. 1(a) and 1(b) are almost equivalent in the linear-response and shear-thinning regimes, where the friction in Eq. (6) is dominated by the memory effects leading to a slowing down of the relaxation. The difference in the microscopic damping $v_{s}$ does not play a significant role. In contrast, for large pulling forces, the correlation function $\phi_{s}(t)$ relaxes to zero rapidly and the integral Eq. (6) is dominated by the short-time part of the correlation functions.

\section{B. Comparison with simulation data}

The simulation is performed in two dimensions and the setup is the same as described in Ref. [15]: In a bidisperse mixture of hard disks with size ratio $R_{s} / R_{b}=4 / 5$ of small to big particles and a respective mass ratio $m_{s} / m_{b}=16 / 25$, an intruder of radius $R_{0}=2 R_{s}$ and mass $m_{0}=4 m_{s}$ is suspended. All collisions are inelastic, characterized by the coefficient of restitution, $\varepsilon$. The particles are kicked randomly to balance energy input and dissipation by drag and inelastic collisions. Lengths and masses are measured such that $R_{s}=1$ and $m_{s}=1$ and a time scale is set by requiring that the granular temperature $T_{G}=1$ in the system with $F_{\mathrm{ex}}=0$. An event driven code is implemented to simulate $N=10^{4}$ particles for a wide range of $F_{\text {ex }}$ and $\varepsilon$.

We adopt with $v_{s}=1+F_{\mathrm{ex}}^{2}$ the second schematic model to compare with the simulation data in detail. Since the schematic models only capture the overall mathematical structure of the theory, it is equally well applicable in both three and two dimensions as the underlying glass transitions are similar in both 2D and 3D [12]. The same holds for monodisperse and bidisperse systems.

Figure 2 shows the fit of the measured correlation functions by the model. The numerical solution of the density autocorrelator of the intruder fits quite well the corresponding simulation data for the moderately high force $F_{\mathrm{ex}}=250$. For the smaller force $F_{\mathrm{ex}}=1$, it shows some deviations. The fitting parameters are $v_{A}=200, \sigma=-0.05$ for $\varepsilon=0.9$ as well as (not shown in Fig. 2) $v_{A}=300, \sigma=-0.09$ for $\varepsilon=0.7$ and $v_{A}=600$,

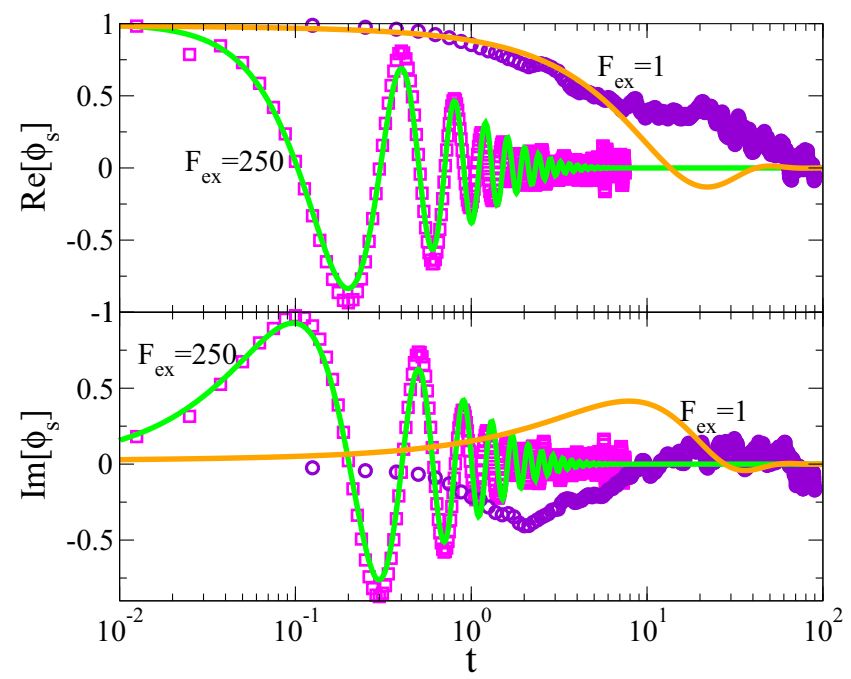

FIG. 2. (Color online) Comparison between the schematic model and the simulation data for the density autocorrelator of the probe particle at fixed packing fraction $\varphi=0.8$ and energy dissipation $\varepsilon=0.9$. The pulling forces are $F_{\mathrm{ex}}=1$ and 250 . The symbols represent the simulation data; the solid lines are the descriptions by the schematic model.

$\sigma=-0.13$ for $\varepsilon=0.1$. The other parameters are the same as the ones mentioned above.

The corresponding fit of the friction coefficients is given in Fig. 3. In the regime of small forces, the schematic model shows a linear-response plateau. The simulation data also show a plateau for small forces (see Fig. 3 in Ref. [15]). As the glass transition is approached, this regime moves to smaller forces, so that it is visible in Fig. 3 only for the smallest $\varepsilon=0.1$, which is further away from the glass transition than $\varepsilon=0.9$ and 0.7 . However, for $\varepsilon=0.1$ and $\varphi=0.8$ the simulations become increasingly difficult for small forces due to the occurrence of long-lasting contacts. Hence, the error bars become comparable to the result itself. For large pulling forces,

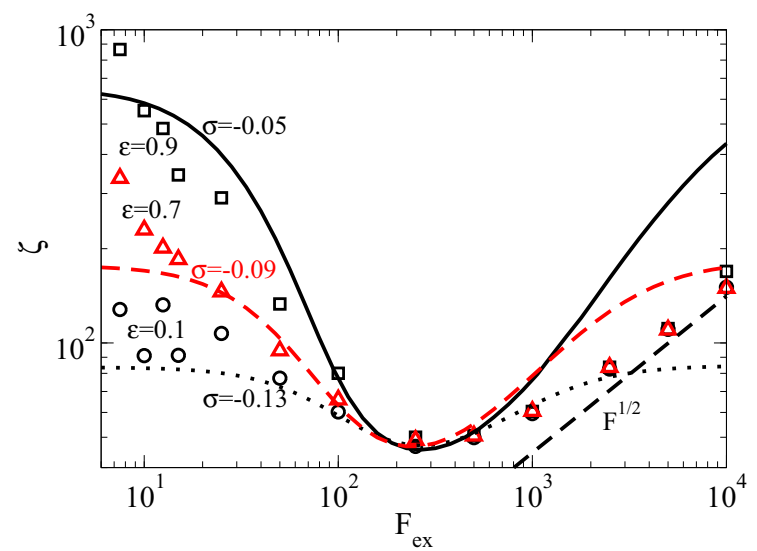

FIG. 3. (Color online) Effective friction of the intruder for different energy dissipation $\varepsilon=0.9,0.7$, and 0.1 at fixed packing fraction $\varphi=0.8$ of the host fluid. Individual data points show the simulation results; curves represent the results from the schematic models. The dashed straight line indicates the slope of $\sqrt{F}$. 
the model shows qualitatively how the increasing friction coefficient can be rationalized within a schematic model. While the schematic model exhibits different limits for varying distances from the glass transition, the simulation data follow the same curve for $F_{\text {ex }} \gtrsim 500$. Between the extreme regimes of large and small pulling forces the friction coefficient exhibits a minimum that is similar for all distances from the glass transition for both the schematic model and the simulation.

While the schematic model may only qualitatively fit the simulation data for the friction coefficient in Fig. 3, in addition to the good agreement of the correlation functions in Fig. 2, the results are also consistent with the predictions from MCT for hard spheres [10-12]. For smaller dissipation, i.e., larger coefficient of restitution $\varepsilon$ in Fig. 3, the data can be described only by choosing points closer to the glass-transition line in the schematic model. Smaller distances to the glass transition given by smaller values of $\sigma$ indicate that for the same density of $\varphi=0.8$ the data for $\varepsilon=0.9$ are much closer to the glass transition than for $\varepsilon=0.1$ with $\varepsilon=0.7$ located in between. This finding is in agreement with the predicted increase of the glass-transition density with decreasing $\varepsilon$ within MCT [10-12].

\section{KINETIC THEORY IN THE LOW-DENSITY LIMIT}

To clarify the origin of the scaling law $\zeta \propto \sqrt{F_{\text {ex }}}$ in the large-force asymptote, we propose a simple kinetic theory in the following. The simulation result from Ref. [15] has shown that the scaling law is independent of packing fraction. Therefore, a potential explanation of the increased friction by jamming or shear thickening seems unlikely. Also, the correlation functions for large pulling forces decay relatively quickly, cf. Fig. 2, also contradicting a buildup of long-time glass-like contributions to the integrals like in Eq. (6). In the following we shall therefore focus on the low-density limit, where exact solutions can be obtained.

The formal solution of $\boldsymbol{v}(t)$ in Eq. (1) can be readily obtained and the corresponding ensemble average of the velocity of the intruder is given by

$$
\langle\boldsymbol{v}(t)\rangle=\frac{\boldsymbol{F}_{\mathrm{ex}}}{\zeta_{0}}\left(1-e^{-\frac{\zeta_{0}}{m} t}\right)+\frac{e^{-\frac{\zeta_{0}}{m} t}}{m} \int_{0}^{t} e^{\frac{\zeta_{0}}{m} t^{\prime}}\left\langle\boldsymbol{f}_{\mathrm{int}}\left(t^{\prime}\right)\right\rangle d t^{\prime},
$$

where we have averaged out the initial velocity and the random force: $\left\langle\boldsymbol{v}_{0}\right\rangle=0$ and $\langle\boldsymbol{\eta}(t)\rangle=0$.

The direct calculation of $\left\langle\boldsymbol{f}_{\text {int }}(t)\right\rangle$ in Eq. (7) is difficult. The key point of our kinetic theory is to introduce the mean free path of the intruder, $l_{0}=\rho^{-1} \sigma_{c r}$, where $\rho=N / V$ is the particle number density and $\sigma_{\mathrm{cr}}$ is the intruder's cross section, which for hard sphere reduces to $\sigma_{\mathrm{cr}}=4 \pi R^{2}$. Let us denote the collision time as $t_{c}$. Between two successive collisions $n t_{c}<t<(n+1) t_{c}$, there is no interaction force in the hard sphere limit, $\left\langle\boldsymbol{f}_{\text {int }}(t)\right\rangle=0$. On average, after $t_{c}$ a collision event causes a momentum transfer from the intruder to its collision partner of the order of the intruder's complete momentum. The velocity of the intruder increases again from almost zero due to the constant pulling force. Statistically, the intruder's velocity exhibits periodic motion. Consider the motion of the probe in the first period: The average velocity reads

$$
\langle\boldsymbol{v}(t)\rangle=\frac{\boldsymbol{F}_{\mathrm{ex}}}{\zeta_{0}}\left(1-e^{-\frac{\zeta_{0}}{m} t}\right), \quad 0 \leqslant t \leqslant t_{c},
$$

and the displacement of the motion satisfies

$$
l_{0}=\left|\int_{0}^{t_{c}}\langle\boldsymbol{v}(t)\rangle d t\right|=\frac{F_{\mathrm{ex}}}{\zeta_{0}}\left[t_{c}-\frac{m}{\zeta_{0}}\left(1-e^{-\frac{\zeta_{0}}{m} t_{c}}\right)\right] .
$$

The average velocity of the probe is given by

$$
\left\langle v_{s}\right\rangle=\frac{1}{t_{c}}\left|\int_{0}^{t_{c}}\langle\boldsymbol{v}(t)\rangle d t\right|=\frac{l_{0}}{t_{c}} .
$$

In general, the friction of the probe $\left\langle v_{s}\right\rangle$ can be calculated by Eqs. (9) and (10) exactly. We first consider the two limiting cases $t_{c} \gg \frac{m}{\zeta_{0}}$ (overdamped limit) and $t_{c} \ll \frac{m}{\zeta_{0}}$ (ballistic regime).

In the overdamped limit, velocity relaxation dominates over collisions and the collision times are large,

$$
t_{c}=\frac{l_{0} \zeta_{0}}{F_{\mathrm{ex}}} \gg \frac{m}{\zeta_{0}}
$$

or equivalently,

$$
\frac{F_{\mathrm{ex}}}{\zeta_{0}^{2}} \ll \frac{l_{0}}{m} .
$$

The average velocity and the friction of the intruder can be obtained by Eq. (10) and definition of the friction itself, yielding

$$
\left\langle v_{s}\right\rangle=F_{\mathrm{ex}} / \zeta_{0}, \quad \zeta=\zeta_{0}
$$

The friction experienced by the intruder is dominated by the effective friction originating from the medium.

In the ballistic limit, collisions dominate over velocity relaxation. Expanding $e^{-\frac{5_{0}}{m} t}$ in Eq. (9) to second order, we get

$$
t_{c}=\sqrt{\frac{2 m l_{0}}{F_{\mathrm{ex}}}} \ll \frac{m}{\zeta_{0}} .
$$

The ballistic limit is given by the presence of pulling forces very large compared to the bare friction,

$$
\frac{F_{\mathrm{ex}}}{\zeta_{0}^{2}} \gg \frac{2 l_{0}}{m} \text {. }
$$

The average velocity and the friction of the probe are

$$
\begin{aligned}
\left\langle v_{s}\right\rangle & =\sqrt{\frac{l_{0} F_{\mathrm{ex}}}{2 m}} \propto \sqrt{F_{\mathrm{ex}}} \\
\zeta & =\sqrt{\frac{2 m F_{\mathrm{ex}}}{l_{0}}} \propto \sqrt{F_{\mathrm{ex}}} .
\end{aligned}
$$

Both the velocity as well as the friction are proportional to the square-root of the external pulling force and independent of the bare friction.

The general solution of Eqs. (9) and (10) can be calculated in parametric form and is given in Fig. 4, where the crossover is shown for the friction coefficient from a constant (linear large-force behavior of the velocity) to the square-root increase (square-root increase of the velocity for large forces). The 


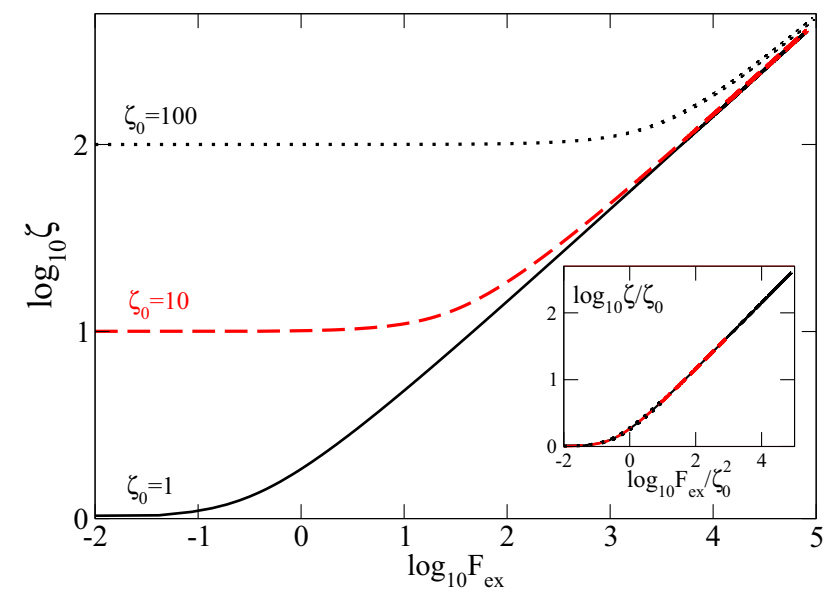

FIG. 4. (Color online) Force-friction relation at large forces for different damping in the low-density limit.

reason why for a driven granular system the friction of the probe increases as $\zeta \propto \sqrt{F_{\mathrm{ex}}}$ in the large-force regime, but for colloidal hard-sphere systems the friction only decreases to a constant value, can be explained as follows. For different bare frictions $\zeta_{0}$, the $F_{\text {ex }}-\zeta$ plots can be rescaled as $F_{\text {ex }} / \zeta_{0}^{2}$ versus $\zeta / \zeta_{0}$, cf. the inset in Fig. 4 . The behavior of the probe in the large-pulling-force regime is determined by the ratio of the collision time scale over the Brownian velocity relaxation time scale, $t_{c} / \frac{m}{\zeta_{0}}$, or equivalently the value of the rescaled force $F_{\text {ex }} m /\left(\zeta_{0}^{2} l_{0}\right)$. In a driven granular system, the bare friction is quite small compared with the one in a Brownian suspension, $\zeta_{0}=1$ in the granular simulation [15] and $\zeta_{0}=50$ in the colloidal one [5]. Indeed, one would also obtain the same asymptotic behavior $\zeta \propto \sqrt{F_{\mathrm{ex}}}$ for Brownian systems for extremely large pulling forces.

\section{CONCLUSION}

We have investigated the microrheology of the driven granular hard-sphere system by a schematic model and a simple kinetic theory. For small and moderate external pulling forces, the schematic model agrees reasonably well with the simulation data, cf. Fig. 3, and implies that the glass-transition density increases with smaller coefficient of restitution $\varepsilon$, confirming predictions from mode-coupling theory [10-12]. For large forces, glassy dynamics becomes irrelevant and a simple kinetic theory clarifies the origin of the scaling of the friction with increasing pulling force. When damping by a surrounding fluid dominates the motion of the intruder at high forces, a second linear regime emerges where the friction becomes constant. When collisions dominate, the friction increases in a square-root law, $\zeta \propto \sqrt{F_{\text {ex }}}$. Such behavior of the drag force, $F_{\mathrm{ex}} \propto v^{2}$, is reminiscent of Bagnold's law for granular rheology where the shear stress is proportional to the square of the shear rate.

While the derivation of the equations above implies the existence of a thermostat to ensure a steady state at the presence of energy dissipation, one may argue that for high enough velocities of the intruder, sufficient energy may be injected into the host fluid to overcome the necessity of an additional thermostat: Consistent with that view, experiments without thermostat but high intruder velocities [19] found for high densities that the average drag force $F$ increased quadratically with the pulling velocity $v$ of the intruder, which is consistent with $\zeta \propto \sqrt{F_{\mathrm{ex}}}$ found above.

In the models presented in this work, the phenomenon of increasing friction $\zeta$ for high pulling forces is not specific to the details of granular dynamics; it may only be more likely to access that high-force regime in granular simulations and experiments for typical control parameters. The elaboration of the microscopic version of the theory similar to the Brownian case [9] shall clarify this issue in the future.

\section{ACKNOWLEDGMENTS}

We acknowledge support from DAAD and DFG within FOR1394. We thank Andrea Fiege, Matthias Fuchs, Till Kranz, Leo Silbert, Thomas Voigtmann, Anoosheh Yazdi, and Peidong Yu for valuable discussions.
[1] T. M. Squires and T. G. Mason, Annu. Rev. Fluid Mech. 42, 413 (2010).

[2] P. Habdas, D. Schaar, A. C. Levitt, and E. R. Weeks, Europhys. Lett. 67, 477 (2004).

[3] A. Meyer, A. Marshall, B. G. Bush, and E. M. Furst, J. Rheol. 50, 77 (2006).

[4] J.-A. Conchello and J. W. Lichtman, Nat. Meth. 2, 920 (2005).

[5] M. V. Gnann, I. Gazuz, A. M. Puertas, M. Fuchs, and T. Voigtmann, Soft Matter 7, 1390 (2011).

[6] D. Winter, J. Horbach, P. Virnau, and K. Binder, Phys. Rev. Lett. 108, 028303 (2012).

[7] D. Winter and J. Horbach, J. Chem. Phys. 138, 12 A512 (2013).

[8] I. Gazuz, A. M. Puertas, T. Voigtmann, and M. Fuchs, Phys. Rev. Lett. 102, 248302 (2009).

[9] I. Gazuz and M. Fuchs, Phys. Rev. E 87, 032304 (2013).

[10] W. T. Kranz, M. Sperl, and A. Zippelius, Phys. Rev. Lett. 104, 225701 (2010).
[11] M. Sperl, W. T. Kranz, and A. Zippelius, Europhys. Lett. 98, 28001 (2012).

[12] W. T. Kranz, M. Sperl, and A. Zippelius, Phys. Rev. E 87, 022207 (2013).

[13] R. Candelier and O. Dauchot, Phys. Rev. Lett. 103, 128001 (2009).

[14] R. Candelier and O. Dauchot, Phys. Rev. E 81, 011304 (2010).

[15] A. Fiege, M. Grob, and A. Zippelius, Granular Matter 14, 247 (2012).

[16] A. Puglisi, A. Baldassarri, and V. Loreto, Phys. Rev. E 66, 061305 (2002).

[17] M. Fuchs and M. E. Cates, Phys. Rev. Lett. 89, 248304 (2002).

[18] W. Götze, Complex Dynamics of Glass-Forming LiquidsA Mode-Coupling Theory (Oxford University Press, Oxford, 2009).

[19] Y. Takehara, S. Fujimoto, and K. Okumura, Europhys. Lett. 92, 44003 (2010). 



\section{Part III.}

\section{Summary and discussion}



In summary, we studied rheological properties of dry granular media. In the following, we recapitulate the key results of the articles.

In section 7.1, we presented a detailed study on the jamming transition of frictional granular media confined in small simulation cells. The main result is a jamming phase diagram of sheared frictional granular media based on a theory for a first-order phase transition. At finite Coulomb friction coefficient, three well separated packing fractions, $\left(\phi_{c}, \phi_{\sigma}, \phi_{\eta}\right)$, describe the rheological behavior:

(i) $\phi_{\mathrm{c}}$ is the onset of rigidity, i.e., a packing fraction above which a finite stress, $\Sigma \neq 0$, can produce shear jammed states,

(ii) $\phi_{\sigma}$ is the lowest packing fraction where a finite yield stress, $\Sigma_{\mathrm{Y}}$, occurs, i.e., for $\phi \geq \phi_{\sigma}$ it holds that $\Sigma(\dot{\gamma} \rightarrow 0, \phi)=\Sigma_{\mathrm{Y}}(\phi)>0$, and

(iii) $\phi_{\eta}$ is the packing fraction where the generalized viscosity, $\eta=\sigma / \dot{\gamma}^{2}$, diverges at zero strain rate and above, for $\phi>\phi_{\eta}$, no inertial flow is possible.

The lowest of the three packing fractions, $\phi_{\mathrm{c}}$, and random loose packing, $\phi_{\mathrm{rlp}}$, are both considered as the onset of rigidity in a frictional packing. We expect $\phi_{\mathrm{c}}$ to equal random loose packing for infinite Coulomb friction parameter Silbert, 2010]. The fact that we can assign a physical meaning to $\phi_{\sigma}$ and $\phi_{\eta}$, is an advancement compared to treating them as "fictitious" or "fitting" parameters for scaling laws Otsuki and Hayakawa 2011 (therein called $\phi_{\mathrm{S}}$ and $\phi_{\mathrm{L}}$, respectively).

Moreover, we observe reentrant flow: Starting from a jammed configuration at finite stress, and then lowering or increasing the imposed stress, leads to flow. Instead of the inertial flow phase at low stress in our reentrance topology, in $\mathrm{Bi}$ et al. 2011, the authors report fragile states, i.e., anisotropic jammed states with force chains, which percolate in only one direction. The difference between the inertial flow phase at low stress and the fragile states stems from the fact that our grains are embedded in a frictionless environment, which allows for ballistic motion and thus inertial flow. In the experiment by Bi et al. 2011, particles, which are subject to quasistatic shear, move on a bottom plate with a finite roughness, i.e., no inertial flow is possible. Fragile states are eliminated when friction with the bottom plate is ruled out Zhang et al. 2015. Another route to fragile states is cohesion between particles Zhang et al., 2015; Irani et al., 2014, 2016. Our simulations neither incorporate friction with a bottom plate nor cohesion, consistent with the absence of fragile states. 
In section 7.2, we studied the rheological response of a frictional granular medium in a large simulation cell. The main result is the existence of strongly time-dependent and heterogeneous states. We showed the existence of unsteady states by prescribing stress in the unstable region of a flow curve. The hydrodynamic model, which is derived in this work, couples hydrodynamics to the evolution of a microstructure variable, which we associate with contributions to the dynamics that stem from friction. The steady state solutions of the hydrodynamic model corresponding to flow equal the flow curves in the previous article, see Grob et al. 2014 and section 7.1. The steady states are investigated with linear stability analysis that is in agreement with the simulation.

In the phase diagram for large granular systems Grob et al., 2016], three packing fractions, $\left(\phi_{\mathrm{c}}, \phi_{\sigma}, \phi_{\eta}\right)$, are necessary to describe the rheology — just as for small simulation cells, as studied in Grob et al. 2014. From the discussion of the phase diagram and the previous study, [Grob et al., 2014], we expect that without the contribution associated with friction, the frictionless scenario is recovered and only steady and homogeneous flow is possible. Unsteady states, either oscillatory or chaotic, have been shown to exist in scalar models Cates et al. 2002. Moreover, experimental findings show unpredictable flow behavior in a potential discontinuous shear thickening regime Hermes et al. 2015. However, this work presents the first example of chaotic flow for a dry granular medium.

Section 7.3 dealt with the characterization of flow states of dense frictional granular matter. It connects the previous studies Grob et al. 2014 and Grob et al. 2016. In both previous studies, we presented a phase diagram. These phase diagrams show an identical sequence of characteristic packing fractions, $\left(\phi_{\mathrm{c}}, \phi_{\sigma}, \phi_{\eta}\right)$. However, the rheology of the frictional granular medium, and thus the phases in these phase diagrams, differ between the two studies. We showed that this difference stems from heterogeneities. The size of the simulation cell allows granular media in only large systems to develop heterogeneities in the unstable region of a flow curve. Heterogeneities imply unsteady flow, which leads to shear thickening in the flow curve. Small systems instead show discontinuous shear thickening in a strain rate controlled setting but jam when driven by an imposed stress in the unstable region of a flow curve. We show that the global stress is related to the width of the nonaffine velocity distribution. This width is maximal in the heterogeneous flow state in which the generalized viscosity is also 
maximal. Stress and the width of the nonaffine velocity distribution follow the same time dependence. Moreover, we report strong spatial correlations between stress and nonaffine velocities. In particular, the strong heterogeneities in the stress fields are accompanied by large gradients in the nonaffine velocity fields.

In addition to the stress, which is measured in an experiment or a simulation, the nonaffine velocity is a key quantity since dissipative contributions to particle interactions are typically velocity dependent. In Olsson [2016], the author points out how rheology is governed by dissipation via the energy balance. In the frictionless system in Olsson, 2016, the width of the velocity distribution increases monotonically as jamming is approached. This increasing width means that the number of particles dominating dissipation decreases. These particles are the fastest particles. Below jamming, we report the fastest particles, which presumably contribute most to dissipation, in the shear thickening regime. Both, flow in the proximity of jamming and shear thickening, are flow regimes that possess a broad nonaffine velocity distribution and, consistently, both are regimes that are governed by heterogeneity and cooperative motion giving rise to enhanced velocities.

In Chapter 8 , the dynamics of an active probe particle near the granular glass transition was probed by numerical simulations and related to analytical results. The steady state behavior of the probe particle provides a velocity-force relation. This velocity-force relation is linear for low forces far from the glass transition. Close to the glass transition, the linear response regime is followed by a superlinear velocity-force dependence. For large forces, we report a sublinear velocity-force relation, independent of the packing fraction. The kinetic theory by Wang and Sperl in Wang et al. 2014 rationalized the sublinear dependence in the granular system.

Experiments show [Habdas et al., 2004] that also colloids display a superlinear velocity-force dependence close to the glass transition. This effect is rationalized by a localization/delocalization transition that takes place when a packing fraction dependent force threshold is crossed [Gazuz and Fuchs, 2013]. The sublinear force thickening regime at large forces, which we observe for granular particles, is in contrast to linear or superlinear regimes in colloidal systems, as observed in experiments Habdas et al., 2004, Wilson et al., 2009, numerical simulations Carpen and Brady, 2005, and predicted by mode coupling theory Gazuz et al. 
2009.

The kinetic theory by Wang and Sperl Wang et al. 2014 also links the microrheology studies and the shear rheology studies together. The theory states that in a collision dominated regime, the friction coefficient grows as a squareroot with the external force: $\zeta \propto F_{\text {ext }}^{1 / 2}$. This implies that the velocity grows as $v \propto F_{\text {ext }}^{1 / 2}$ or $v^{2} \propto F_{\text {ext }}$ - analogous to Bagnold's results for shear rheology studies, where the only relevant time scale of the inertial flow regime is set by the strain rate Bagnold, 1954. In both cases, the inertial flow in shear rheology and the limit of large driving forces in microrheology, the dynamics is governed by collisions of hard grains. Therefore, the only relevant time scale is the external time scale, i.e., $\dot{\gamma}$ in shear rheology or $F_{\text {ext }}$ in microrheology.

The other flow regimes of the shear- and microrheology studies cannot be connected directly. The shear rheology studies are carried out with particles with a finite elasticity, which sets a time scale and allows for plastic flow. This timescale is absent in the microrheology studies since the particles are infinitely hard. If they possessed a large (but finite) stiffness the collisional regime would be followed by elastic-inertial flow at a driving force once particle deformations would become important. The stochastic agitation of the bath particles in the microrheology studies sets a time scale that leads to an effective granular temperature and thus diffusion. This time scale is absent in our shear rheology studies, which exclude flow regimes other than quasistatic or inertial flow at low strain rate.

In essence, this thesis provided (i) a thorough study of the jamming phenomenon of dry frictional particles and (ii) the examination of the dynamics of a probe particle in a granular medium close to the granular glass transition. Both, shear rheology and microrheology, treated transition phenomena of nonequilibrium systems. In the shear rheology study, we presented results that rely on the presence of friction. The incorporation of friction increases the complexity of the jamming scenario but provides a stronger connection to experimental results. We provide a shear thickening and shear jamming scenario of a dry granular system and report, for the first time, unsteady flow states. The microrheology studies revealed the role of the different time scales whose interplay determines the dynamics of a probe particle by which the rheological properties of its surrounding are characterized. Overall, this thesis sheds light on fundamental aspects of the peculiarities of particulate media rheology. 
Part IV.

Outlook 

The work presented in this thesis contributes to a better understanding of the flow of dry frictional particles under simple shear. Three major future directions are directly implied by this work:

(i) the investigation of the microscopic processes,

(ii) the characterization of the chaotic nature of the unsteady flow,

(iii) and the study of frictional particles in Poiseuille flow geometry.

In the following, we discuss the directions (i)-(iii) in detail.

On a microscopic scale, friction opens two new channels of energy dissipation: in addition to normal viscous damping, particles can rotate and dissipate energy during sliding or non-sliding interactions. The role played by different dissipation mechanisms is crucial for the flow of granular media [DeGiuli et al., 2015]. As show in this thesis, the transition from flowing states into jammed states is also highly affected by friction. When a frictional granular medium jams, all the contacts become non-sliding since sliding is a dynamic phenomenon. For infinite friction coefficient, however, all contacts are non-sliding, irrespective of the state of the granular medium. In contrast, in the frictionless limit, all contacts slide but jamming at finite $\Sigma$ below $\phi_{\mathrm{J}}$ is not possible. The role of sliding contacts and dissipation via sliding in the (shear) jamming picture remains elusive. Moreover, the relation between sliding and shear transformations - plastic, dissipative, and irreversible events - is not yet studied.

It is challenging to detect the chaotic nature of the unsteady flow. Several algorithms exist that allow for the computation of Lyapunov exponents of a dynamical system based on a time series of a signal, see, e.g., Sano and Sawada, 1985; Geist et al., 1990; Rosenstein et al., 1993; Kantz, 1994; Small et al., 2013. For an accurate estimate of the Lyapunov exponent the time series needs to be sufficiently long but with high resolution. In addition, a zoo of (technical) parameters needs to be tuned according to the dynamics. The presence of mechanical noise, which stems from the collisions of grains, makes a distinction between mechanical noise and chaotic dynamics a delicate problem. An unusual path in studying the jamming transition is taken in Banigan et al. 2013. There, the authors apply techniques of nonlinear dynamics to molecular dynamics simulations close to the jamming transition and report not only a transition from liquid to solid state near $\phi_{\mathrm{J}}$ but also from chaotic to non-chaotic dynamics when the strain 


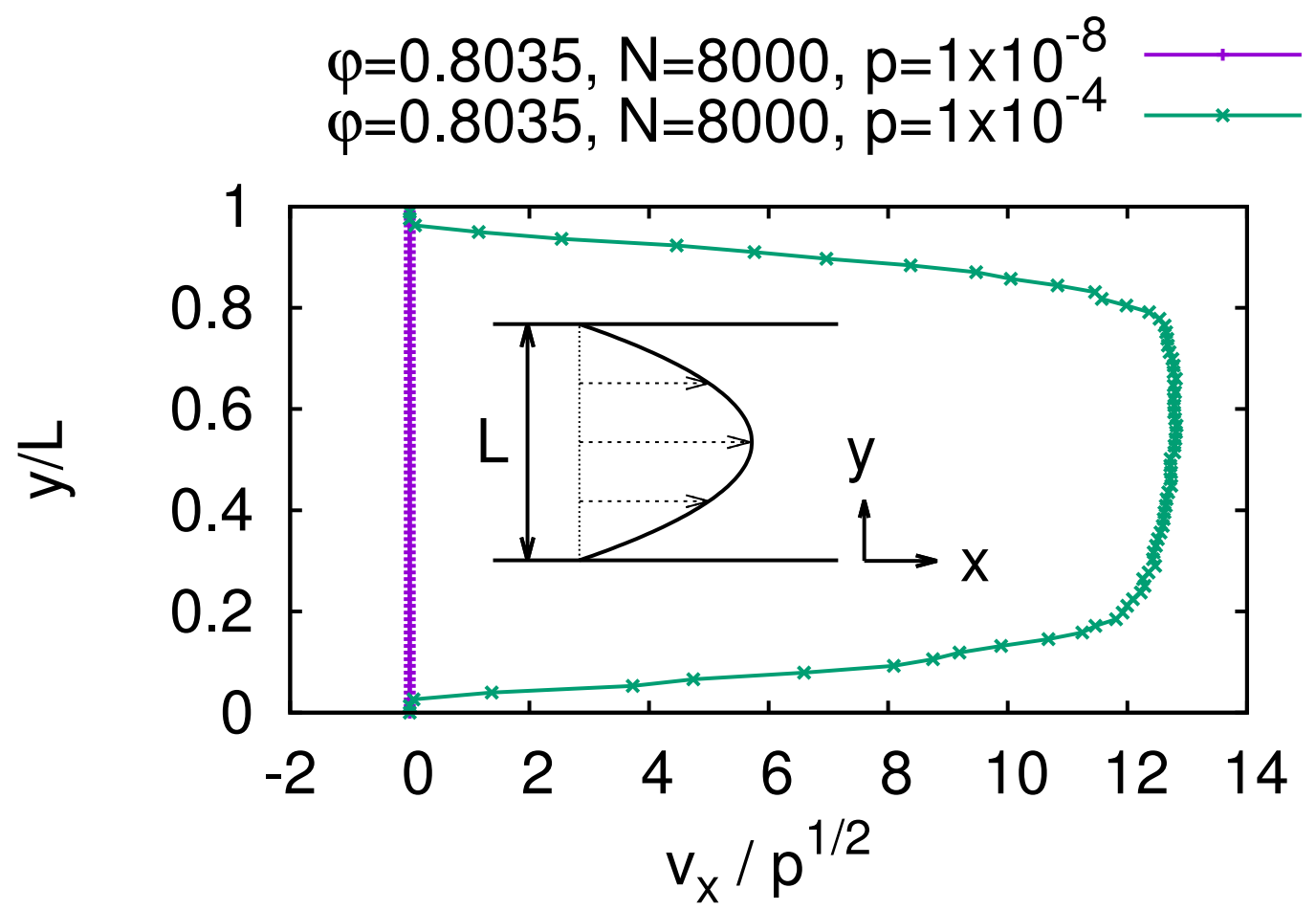

Figure 8.1.: Inset: Poiseuille flow geometry with suggested parabolic velocity profile. Main: Non-parabolic profile of streaming velocity, $v_{x}$, showing flow (large pressure, green) and arrest (low pressure, purple). Units according to Grob et al. 2014.

rate of the system is fixed. The dependence of the largest Lyapunov exponent on the packing fraction suggests that a non-chaotic regime exists at low packing fractions far from the jamming transition, i.e., in the inertial flow regime.

Poiseuille flow, i.e., the flow through a pipe, is a natural step in the direction of the investigation of granular media under shear. Simple shear has the conceptual advantage that hydrodynamics implies homogeneous strain rate and stress profiles in a steady state. In contrast, a Newtonian fluid flowing through a pipe shows a non-homogeneous steady state: The velocity profile is a parabola, see inset of figure 8.1. Therefore, we expect that the strain rate profile and the stress profile in a granular fluid are non-homogeneous, too. Figure 8.1 shows a set of velocity profiles that show non-parabolic velocity fields and a clear distinction between flowing and non-flowing states. These phenomena have already been reported in studies of particulate media, see, e.g., Varnik and Raabe, 2008, Chikkadi 
and Alam, 2009, Chaudhuri and Horbach, 2013, but frictional effects have not yet been taken into account. In simple shear geometry, steady state flow is homogeneous and probes one point in our proposed phase diagrams. In contrast, Poiseuille flow is inherently heterogeneous and samples not only a point in the phase diagram but a line, e.g., at constant packing fraction but ranging over an interval of stress. It would be interesting to study whether Poiseuille flow exhibit several coexisting phases with different stress at the same time, i.e., blobs of shear jammed particles and areas of a flowing phase, either in plastic or inertial flow. 



\section{Part V. \\ Appendix}





\section{A. Details on the hydrodynamic model}

In this Chapter, we detail the stability analysis, of the model that we present in the article "Rheological chaos of frictional grains" in Chapter 7.2. Here, we use the second equation in equations 2 in Grob et al., 2016,

$$
\dot{\gamma}(\sigma, w)=\dot{\gamma}_{0}(\sigma)-w
$$

and formulate the model, equations 2 from Grob et al. 2016], via two equations:

$$
\begin{aligned}
\partial_{t}\left(\dot{\gamma}_{0}(\sigma)-w\right) & =\partial_{y}^{2} \sigma \\
\partial_{t} w & =-\frac{\dot{\gamma}}{\Gamma}\left(w-w^{*}\right)
\end{aligned}
$$

The microstructure variable, $w$, evolves towards a stress dependent value: $w^{*}=$ $w^{*}(\sigma)$. As $w$ describes frictional contributions, $w^{*}$ should be zero at zero stress and it should grow with the stress that is applied. The simplest relation which fulfills these requirements is $w^{*}(\sigma)=b \sigma$.

The model allows for two stationary states, i.e., the left hand sides of equations A.2 are set to zero. In a stationary state, $\left(\sigma_{0}, w_{0}\right)$, the stress is homogeneous and $w$ determines the strain rate via equation A.1. The first solution corresponds to stationary flow:

$$
w_{0}=w^{*}(\sigma) ; \quad \sigma_{0}=\sigma .
$$

The flowing steady state solution A.3 recovers the phenomenological constitutive equation, equation 1 in article Grob et al. 2014, via equation A.1. This implies for the slope

$$
\left.\partial_{\sigma} \dot{\gamma}\right|_{\sigma_{0}}=\left.\partial_{\sigma} \dot{\gamma}_{0}\right|_{\sigma_{0}}-b
$$


A. Details on the hydrodynamic model

The second stationary solution accounts for the shear jammed state:

$$
w_{0}=\dot{\gamma}_{0}\left(\sigma_{0}\right) ; \quad \sigma_{0}=\sigma
$$

In this solution, inserting equation A.5 in equation A.1 leads to zero strain rate.

In the following, we discuss the stability of the solutions in equations A.3 and A.5. We employ linear stability analysis and consider small deviations, $\delta \sigma=\sigma-$ $\sigma_{0}$ and $\delta w=w-w_{0}$, from the steady state solution, $\left(\sigma_{0}, w_{0}\right)$, of the equation A.2.

\section{A.1. The flowing state}

The linearization of the coupled equations A.2 requires the linearization of each term. To linearize around the flowing solution, equation A.3, we linearize the frictionless constitutive relation, $\dot{\gamma}_{0}(\sigma)$, and its derivative: $\dot{\gamma}_{0}\left(\sigma_{0}+\delta \sigma\right)=\dot{\gamma}_{0}\left(\sigma_{0}\right)+$ $\left(\frac{1}{2} a \sigma^{-1 / 2}+2 c \sigma\right) \delta \sigma+\mathcal{O}\left(\delta \sigma^{2}\right)$ and $\partial_{\sigma} \dot{\gamma}_{0}(\sigma)=\partial_{\sigma} \dot{\gamma}_{0}\left(\sigma_{0}\right)-\left(\frac{1}{4} a \sigma_{0}^{-3 / 2}+2 c\right) \delta \sigma+\mathcal{O}\left(\delta \sigma^{2}\right)$, respectively. Up to linear order in the small quantities, $\delta \sigma$ and $\delta w$, or products of these, and with the notation $\tau=\Gamma / \dot{\gamma}\left(\sigma_{0}\right)$ equation A.2 reads:

$$
\begin{aligned}
\partial_{y y} \delta \sigma-\left.\partial_{\sigma} \dot{\gamma}_{0}\right|_{\sigma_{0}} \partial_{t} \delta \sigma+\partial_{t} \delta w & =0 \\
\frac{b}{\tau} \delta \sigma-\frac{1}{\tau} \delta w-\partial_{t} \delta w & =0
\end{aligned}
$$

By Fourier transform, we substitute $\partial_{y} \rightarrow i k$, with wave number $k$, and $\partial_{t} \rightarrow$ $i \omega:=\Omega$, with frequency, $\omega$ or growth rate $\Omega$. The Fourier transform of equations A.6 can be written in matrix notation:

$$
\left(\begin{array}{l}
0 \\
0
\end{array}\right)=\left(\begin{array}{cc}
-k^{2}-\left.\partial_{\sigma} \dot{\gamma}_{0}\right|_{\sigma_{0}} \Omega & \Omega \\
\frac{b}{\tau} & -\frac{1}{\tau}-\Omega
\end{array}\right)\left(\begin{array}{c}
\hat{\delta \sigma} \\
\hat{\delta w}
\end{array}\right) .
$$

The hat indicates the transformed variables. If the determinant of the matrix in equation A.7 vanishes there can be more than the trivial solution $(\delta \sigma=0=\delta w)$. Hence, we have

$$
0=\Omega^{2}+\Omega \frac{1}{\left.\tau \partial_{\sigma} \dot{\gamma}_{0}\right|_{\sigma_{0}}}\left(\tau k^{2}+\left.\partial_{\sigma} \dot{\gamma}_{0}\right|_{\sigma_{0}}-b\right)+\frac{k^{2}}{\left.\tau \partial_{\sigma} \dot{\gamma}_{0}\right|_{\sigma_{0}}} .
$$

The polynomial is solved for its roots given all the constants. The roots lead to 
the dispersion relation $\Omega(k)=\Omega^{\prime}+i \Omega^{\prime \prime}$ with

$$
\begin{aligned}
\Omega_{1 / 2}= & -\frac{1}{2} \frac{1}{\left.\tau \partial_{\sigma} \dot{\gamma}_{0}\right|_{\sigma_{0}}}\left(\tau k^{2}+\left.\partial_{\sigma} \dot{\gamma}\right|_{\sigma_{0}}\right) \\
& \pm \sqrt{\left(\frac{1}{2} \frac{1}{\left.\tau \partial_{\sigma} \dot{\gamma}_{0}\right|_{\sigma_{0}}}\left(\tau k^{2}+\left.\partial_{\sigma} \dot{\gamma}\right|_{\sigma_{0}}\right)\right)^{2}-\frac{k^{2}}{\left.\tau \partial_{\sigma} \dot{\gamma}_{0}\right|_{\sigma_{0}}}}
\end{aligned}
$$

A finite coulomb friction parameter, $\mu>0$, i.e., $b>0$, is necessary for the growth of perturbations and oscillations since only then the growth rate, $\operatorname{Re}(\Omega)$, can become positive due to a negative slope, $\left.\partial_{\sigma} \dot{\gamma}\right|_{\sigma_{0}}$, of the flow curve. In the frictionless case, i.e., when $b=0$, the dispersion relation is always negative giving rise to a negative growth rate and stable flow. When $\tau$ gets small, i.e., infinitely fast relaxation of $w$ to $w^{\star}(\sigma)$, or $k \rightarrow 0$, i.e., infinitely large systems, the dispersion relation becomes proportional to the slope of the frictional flow curve, which makes the frictional contribution to the slope, $-b$, become increasingly important.

\section{A.2. The shear jammed state}

A linearization of the coupled equations A.2 around the shear jammed solution, equation A.5, yields up to linear order

$$
\begin{aligned}
\partial_{y y} \delta \sigma-\left.\partial_{\sigma} \dot{\gamma}_{0}\right|_{\sigma_{0}} \partial_{t} \delta \sigma+\partial_{t} \delta w & =0 \\
-\frac{1}{\Gamma}\left(w_{0}-b \sigma_{0}\right)\left(\left.\partial_{\sigma} \dot{\gamma}_{0}\right|_{\sigma_{0}} \delta \sigma-\delta w\right)-\partial_{t} \delta w & =0 .
\end{aligned}
$$

The Fourier transformed set of equations A.10 can be written in matrix notation:

$$
\left(\begin{array}{l}
0 \\
0
\end{array}\right)=\left(\begin{array}{cc}
-k^{2}-\left.\partial_{\sigma} \dot{\gamma}_{0}\right|_{\sigma_{0}} \Omega & \Omega \\
\left.\partial_{\sigma} \dot{\gamma}_{0}\right|_{\sigma_{0}} & -1+\Omega \frac{\Gamma}{w_{0}-b \sigma_{0}}
\end{array}\right)\left(\begin{array}{c}
\hat{\delta \sigma} \\
\hat{\delta w}
\end{array}\right) .
$$

As above, if the determinant of the matrix in equation A.11 vanishes there can be more than the trivial solution $(\delta \sigma=0=\delta w)$. Hence, we have

$$
0=\Omega^{2}+\Omega k^{2} \frac{1}{\left.\partial_{\sigma} \dot{\gamma}_{0}\right|_{\sigma_{0}}}-k^{2} \frac{w_{0}-b \sigma_{0}}{\left.\Gamma \partial_{\sigma} \dot{\gamma}_{0}\right|_{\sigma_{0}}} .
$$

The solutions of equation A.12 lead to the dispersion relation of the shear jammed 
A. Details on the hydrodynamic model

state:

$$
\Omega_{1 / 2}=-\frac{1}{2} \frac{k^{2}}{\left.\partial_{\sigma} \dot{\gamma}_{0}\right|_{\sigma_{0}}} \pm \sqrt{\left(\frac{1}{2} \frac{k^{2}}{\left.\partial_{\sigma} \dot{\gamma}_{0}\right|_{\sigma_{0}}}\right)^{2}+k^{2} \frac{w_{0}-b \sigma_{0}}{\left.\Gamma \partial_{\sigma} \dot{\gamma}_{0}\right|_{\sigma_{0}}}} .
$$

Below $\phi_{\sigma}$, see Grob et al., 2014, 2016], there is potentially one unstable and one stable mode when $w_{0}-b \sigma_{0}$ is positive and large enough, i.e., $\operatorname{Re}\left(\Omega_{1}\right)>0$ and $\operatorname{Re}\left(\Omega_{2}\right)<0$. Shear jamming is then unstable and a flowing solution is attained. Above $\phi_{\sigma}$, the strain rate gets negative and both solutions are stable when $w_{0}-b \sigma_{0}<0$. This is what we identify with shear jamming. Note that here, in contrast to the flowing state, the parameter $b$ enters via the full flow curve, $w_{0}-b \sigma_{0}=\dot{\gamma}_{0}\left(\sigma_{0}\right)-b \sigma_{0}$, and not via the slope. 


\section{Bibliography}

Aharonov, E. and Sparks, D. Rigidity phase transition in granular packings. Physical Review E 60:6890 (1999). http://dx.doi.org/10.1103/PhysRevE.60.6890

Andreotti, B., Barrat, J.-L., and Heussinger, C. Shear flow of non-Brownian suspensions close to jamming. Physical Review Letters 109:105901 (2012). http://dx.doi.org/10.1103/PhysRevLett.109.105901

Aranson, I. S., Tsimring, L. S., Malloggi, F., and Clément, É. Nonlocal rheological properties of granular flows near a jamming limit. Physical Review E 78:031303 (2008). http://dx.doi.org/10.1103/PhysRevE.78.031303

Argon, A. S. Plastic deformation in metallic glasses. Acta Metallurgica 27:47 (1979). http://dx.doi.org/10.1016/0001-6160(79)90055-5

Bagnold, R. A. Experiments on a Gravity-Free Dispersion of Large Solid Spheres in a Newtonian Fluid under Shear. Proceedings of the Royal Society A: Mathematical, Physical and Engineering Sciences 225:49 (1954). http://dx.doi.org/10.1098/rspa.1954.0186

Banigan, E. J., Illich, M. K., Stace-Naughton, D. J., and Egolf, D. a. The chaotic dynamics of jamming. Nature Physics 9:288 (2013).

http://dx.doi.org/10.1038/nphys2593

Baran, O., Ertaş, D., Halsey, T. C., Grest, G. S., and Lechman, J. B. Velocity correlations in dense gravity-driven granular chute flow. Physical Review E 74:051302 (2006). http://dx.doi.org/10.1103/PhysRevE.74.051302

Barker, T., Schaeffer, D. G., Bohorquez, P., and Gray, J. M. N. T. Well-posed and ill-posed behaviour of the mu(I)-rheology for granular flow. Journal of Fluid Mechanics 779:794 (2015). http://dx.doi.org/10.1017/jfm.2015.412

Bashkirtseva, I. A., Zubarev, A. Y., Iskakova, L. Y., and Ryashko, L. B. On rheophysics of high-concentrated suspensions. Colloid Journal 71:446 (2009). http://dx.doi.org/10.1134/S1061933X09040024 
Bibliography

Berret, J.-F., Lerouge, S., and Decruppe, J.-P. Kinetics of the Shear-Thickening Transition Observed in Dilute Surfactant Solutions and Investigated by Flow Birefringence. Langmuir 18:7279 (2002). http://dx.doi.org/10.1021/la011471h

Berret, J.-F. and Porte, G. Metastable versus unstable transients at the onset of a shear-induced phase transition. Physical review. E 60:4268 (1999). http://dx.doi.org/10.1103/PhysRevE.60.4268

Bi, D., Henkes, S., Daniels, K. E., and Chakraborty, B. The Statistical Physics of Athermal Materials. Annual Review of Condensed Matter Physics 6:63 (2015). http://dx.doi.org/10.1146/annurev-conmatphys-031214-014336

Bi, D., Zhang, J., Chakraborty, B., and Behringer, R. P. Jamming by shear. Nature 480:355 (2011). http://dx.doi.org/10.1038/nature10667

Bocquet, L., Colin, A., and Ajdari, A. Kinetic theory of plastic flow in soft glassy materials. Physical Review Letters 103:036001 (2009). http://dx.doi.org/10.1103/PhysRevLett.103.036001

Bonnoit, C., Lanuza, J., Lindner, A., and Clément, É. Mesoscopic length scale controls the rheology of dense suspensions. Physical Review Letters 105:108302 (2010). http://dx.doi.org/10.1103/PhysRevLett.105.108302

Bouzid, M., Izzet, A., Trulsson, M., Clément, E., Claudin, P., and Andreotti, B. Non-local rheology in dense granular flows. The European Physical Journal E 38:1 (2015). http://dx.doi.org/10.1140/epje/i2015-15125-1

Bouzid, M., Trulsson, M., Claudin, P., Clément, É., and Andreotti, B. Nonlocal rheology of granular flows across yield conditions. Physical Review Letters 111:238301 (2013). http://dx.doi.org/10.1103/PhysRevLett.111.238301

Brilliantov, N. V. and Pöschel, T. Kinetic theory of granular gases. Oxford University Press (2010). ISBN 9780198530381. http://dx.doi.org/10.1093/acprof :oso/9780198530381.001.0001

Brown, E. and Jaeger, H. M. Dynamic jamming point for shear thickening suspensions. Physical Review Letters 103:086001 (2009). http://dx.doi.org/10.1103/PhysRevLett.103.086001

Brown, R. Mikroskopische Beobachtungen über die im Pollen der Pflanzen enthaltenen Partikeln, und über das allgemeine Vorkommen activer Molecüle in 
Bibliography

organischen und unorganischen Körpern. Annalen der Physik 90:294 (1828). http://dx.doi.org/10.1002/andp.18280901016

Campbell, C. S. Granular shear flows at the elastic limit. Journal of Fluid Mechanics 465:261 (2002). http://dx.doi.org/10.1017/S002211200200109X

Campbell, C. S. Granular material flows - An overview. Powder Technology 162:208 (2006).

http://dx.doi.org/10.1016/j.powtec.2005.12.008

Campbell, C. S. Clusters in dense-inertial granular flows. Journal of Fluid Mechanics 687:341 (2011).

http://dx.doi.org/10.1007/s10035-014-0521-5

Candelier, R. and Dauchot, O. Creep Motion of an Intruder within a Granular Glass Close to Jamming. Physical Review Letters 103:128001 (2009). http://dx.doi.org/10.1103/PhysRevLett.103.128001

Candelier, R. and Dauchot, O. Journey of an intruder through the fluidization and jamming transitions of a dense granular media. Physical Review E 81:011304 (2010). http://dx.doi.org/10.1103/PhysRevE.81.011304

Carpen, I. C. and Brady, J. F. Microrheology of colloidal dispersions by Brownian dynamics simulations. Journal of Rheology 49:1483 (2005). http://dx.doi.org/10.1122/1.2085174

Cates, M. E., Head, D., and Ajdari, A. Rheological chaos in a scalar shearthickening model. Physical Review E 66:025202 (2002). http://dx.doi.org/10.1103/PhysRevE.66.025202

Cates, M. E., Wittmer, J., Bouchaud, J.-P., and Claudin, P. Jamming, Force Chains and Fragile Matter. Physical Review Letters 81:1841 (1998). http://dx.doi.org/10.1103/PhysRevLett.81.1841

Chaudhuri, P., Berthier, L., and Sastry, S. Jamming transitions in amorphous packings of frictionless spheres occur over a continuous range of volume fractions. Physical Review Letters 104:165701 (2010). http://dx.doi.org/10.1103/PhysRevLett.104.165701

Chaudhuri, P. and Horbach, J. Onset of flow in a confined colloidal glass under an imposed shear stress. Physical Review E 88:040301 (2013). http://dx.doi.org/10.1103/PhysRevE.88.040301 
Bibliography

Chen, L. B., Zukoski, C. F., Ackerson, B. J., Hanley, H. J. M., Straty, G. C., Barker, J., and Glinka, C. J. Structural changes and orientaional order in a sheared colloidal suspension. Physical Review Letters 69:688 (1992). http://dx.doi.org/10.1103/PhysRevLett.69.688

Chialvo, S., Sun, J., and Sundaresan, S. Bridging the rheology of granular flows in three regimes. Physical Review E 85:021305 (2012). http://dx.doi.org/10.1103/PhysRevE.85.021305

Chikkadi, V. and Alam, M. Slip velocity and stresses in granular Poiseuille flow via event-driven simulation. Physical Review E 80:021303 (2009). http://dx.doi.org/10.1103/PhysRevE.80.021303

Ciamarra, M. P., Pastore, R., Nicodemi, M., and Coniglio, A. Jamming phase diagram for frictional particles. Physical Review E 84:041308 (2011). http://dx.doi.org/10.1103/PhysRevE.84.041308

Cundall, P. A. and Strack, O. D. L. A discrete numerical model for granular assemblies. Géotechnique 29:47 (1979). http://dx.doi.org/10.1680/geot.1979.29.1.47

Da Cruz, F. Ecoulement de grains secs : Frottement et blocage. Ph.D. thesis, Ecole Nationale des Ponts et Chaussees (2004).

Da Cruz, F., Emam, S., Prochnow, M., Roux, J. N., and Chevoir, F. Rheophysics of dense granular materials: Discrete simulation of plane shear flows. Physical Review E 72:021309 (2005). http://dx.doi.org/10.1103/PhysRevE.72.021309

de Gennes, P. Granular matter: a tentative view. Reviews of Modern Physics 71:S374 (1999). http://dx.doi.org/10.1103/RevModPhys.71.S374

DeGiuli, E., McElwaine, J. N., and Wyart, M. A Phase Diagram Unifies Energy Dissipation, Kinetics, and Rheology in Inertial Granular Flows. arXiv (2015). http://arxiv.org/abs/1509.03512

Denn, M. M. and Morris, J. F. Rheology of non-brownian suspensions. Annual review of chemical and biomolecular engineering 5:203 (2014). http://dx.doi.org/10.1146/annurev-chembioeng-060713-040221

Dinkgreve, M., Paredes, J., Michels, M. A. J., and Bonn, D. Universal rescaling of flow curves for yield-stress fluids close to jamming. Physical Review E 92:012305 (2015). http://dx.doi.org/10.1103/PhysRevE.92.012305 
Einstein, A. Über die von der molekularkinetischen Theorie der Wärme geforderte Bewegung von in ruhenden Flüssigkeiten suspendierten Teilchen. Annalen der Physik 322:549 (1905). http://dx.doi.org/10.1002/andp.19053220806

Etsion, I. Revisiting the Cattaneo-Mindlin Concept of Interfacial Slip in Tangentially Loaded Compliant Bodies. Journal of Tribology 132:20801 (2010). http://dx.doi.org/10.1115/1.4001238

Falk, M. L. and Langer, J. S. Dynamics of Viscoplastic Deformation in Amorphous Solids. Physical Review E 57:7192 (1997). http://dx.doi.org/10.1103/PhysRevE.57.7192

Fall, A., Bertrand, F., Hautemayou, D., Mezière, C., Moucheront, P., Lemaître, A., and Ovarlez, G. Macroscopic discontinuous shear thickening versus local shear jamming in cornstarch. Physical Review Letters 114:098301 (2015). http://dx.doi.org/10.1103/PhysRevLett.114.098301

Fall, A., Huang, N., Bertrand, F., Ovarlez, G., and Bonn, D. Shear thickening of cornstarch suspensions as a reentrant jamming transition. Physical Review Letters 100:018301 (2008). http://dx.doi.org/10.1103/PhysRevLett.100.018301

Fernandez, N., Mani, R., Rinaldi, D., Kadau, D., Mosquet, M., Lombois-Burger, H., Cayer-Barrioz, J., Herrmann, H. J., Spencer, N. D., and Isa, L. Microscopic mechanism for shear thickening of non-brownian suspensions. Physical Review Letters 111:108301 (2013). http://dx.doi.org/10.1103/PhysRevLett.111.108301

Fernández, V. V. a., Tepale, N., Álvarez, J. G., Pérez-López, J. H., Macías, E. R., Bautista, F., Pignon, F., Rharbi, Y., Gámez-Corrales, R., Manero, O., Puig, J. E., and Soltero, J. F. a. Rheology of the Pluronic P103/water system in a semidilute regime: Evidence of nonequilibrium critical behavior. Journal of Colloid and Interface Science 336:842 (2009). http://dx.doi.org/10.1016/j.jcis.2009.02.064

Fiege, A., Grob, M., and Zippelius, A. Dynamics of an intruder in dense granular fluids. Granular Matter 14:247 (2012). http://dx.doi.org/10.1007/s10035-011-0309-9

Fischer, P. Time dependent flow in equimolar micellar solutions: transient behaviour of the shear stress and first normal stress difference in shear induced structures coupled with flow instabilities. Rheologica Acta 39:234 (2000). http://dx.doi.org/10.1007/s003970000087 
Bibliography

Gazuz, I. and Fuchs, M. Nonlinear microrheology of dense colloidal suspensions: A mode-coupling theory. Physical Review E 87:032304 (2013). http://dx.doi.org/10.1103/PhysRevE.87.032304

Gazuz, I., Puertas, a. M., Voigtmann, T., and Fuchs, M. Active and nonlinear microrheology in dense colloidal suspensions. Physical Review Letters 102:248302 (2009). http://dx.doi.org/10.1103/PhysRevLett.102.248302

Geist, K., Parlitz, U., and Lauterborn, W. Comparison of Different Methods for Computing Lyapunov Exponents. Progress of Theoretical Physics 83:875 (1990). http://dx.doi.org/10.1143/PTP.83.875

Gerl, F. and Zippelius, A. Coefficient of restitution for elastic disks. Physical Review E 59:2361 (1999). http://dx.doi.org/10.1103/PhysRevE.59.2361

Goodrich, C. P., Dagois-Bohy, S., Tighe, B. P., van Hecke, M., Liu, A. J., and Nagel, S. R. Jamming in finite systems: Stability, anisotropy, fluctuations, and scaling. Physical Review E 90:022138 (2014).

http://dx.doi.org/10.1103/PhysRevE.90.022138

Goodrich, C. P., Liu, A. J., and Nagel, S. R. Finite-Size Scaling at the Jamming Transition. Physical Review Letters 109:095704 (2012). http://dx.doi.org/10.1103/PhysRevLett.109.095704

Goyon, J., Colin, A., Ovarlez, G., Ajdari, A., and Bocquet, L. Spatial cooperativity in soft glassy flows. Nature 454:84 (2008). http://dx.doi.org/10.1038/nature07026

Grob, M., Heussinger, C., and Zippelius, A. Jamming of frictional particles: a nonequilibrium first-order phase transition. Physical Review E 89:050201 (2014). http://dx.doi.org/10.1103/PhysRevE.89.050201

Grob, M., Zippelius, A., and Heussinger, C. Rheological chaos of frictional grains. Physical Review E 93:030901 (2016). http://dx.doi.org/10.1103/PhysRevE.93.030901

Güttler, C., Heißelmann, D., Blum, J., and Krijt, S. Normal Collisions of Spheres: A Literature Survey on Available Experiments. arXiv (2012). http://arxiv.org/abs/1204.0001

Guy, B. M., Hermes, M., and Poon, W. C. K. Towards a Unified Description of the Rheology of Hard-Particle Suspensions. Physical Review Letters 115:088304 
Bibliography

(2015).

http://dx.doi.org/10.1103/PhysRevLett.115.088304

Habdas, P., Schaar, D., Levitt, A. C., and Weeks, E. R. Forced motion of a probe particle near the colloidal glass transition. Europhysics Letters 67:477 (2004). http://dx.doi.org/10.1209/epl/i2004-10075-y

Hatano, T. Critical scaling of granular rheology. Progress of Theoretical Physics Supplement 184:143 (2010).

http://dx.doi.org/10.1143/PTPS.184.143

Head, D., Ajdari, A., and Cates, M. E. Jamming, hysteresis, and oscillation in scalar models for shear thickening. Physical Review E 64:061509 (2001). http://dx.doi.org/10.1103/PhysRevE.64.061509

Head, D., Ajdari, A., and Cates, M. E. Rheological instability in a simple shearthickening model. Europhysics Letters 57:120 (2002). http://dx.doi.org/10.1209/epl/i2002-00550-y

Henkes, S., Quint, D. A., Fily, Y., and Schwarz, J. M. Rigid Cluster Decomposition Reveals Criticality in Frictional Jamming. Physical Review Letters 116:028301 (2016). http://dx.doi.org/10.1103/PhysRevLett.116.028301

Hermes, M., Guy, B. M., Poy, G., Cates, M. E., Wyart, M., and Poon, W. C. K. Unsteady flow and particle migration in dense, non-Brownian suspensions. arXiv (2015). http://arxiv.org/abs/1511.08011

Hertz, H. Ueber die Berührung fester elastischer Körper. Journal fur die Reine und Angewandte Mathematik 1882:156 (1882). http://dx.doi.org/10.1515/crll.1882.92.156

Hess, O., Goddard, C., and Hess, S. From shear-thickening and periodic flow behavior to rheo-chaos in nonlinear Maxwell-model fluids. Physica A 366:31 (2006). http://dx.doi.org/10.1016/j.physa.2005.10.007

Heussinger, C. Shear thickening in granular suspensions: Interparticle friction and dynamically correlated clusters. Physical Review E 88:050201 (2013). http://dx.doi.org/10.1103/PhysRevE.88.050201

Heussinger, C. and Barrat, J.-L. Jamming transition as probed by quasistatic shear flow. Physical Review Letters 102:218303 (2009). http://dx.doi.org/10.1103/PhysRevLett.102.218303 
Bibliography

Heussinger, C., Berthier, L., and Barrat, J.-L. Superdiffusive, heterogeneous, and collective particle motion near the fluid-solid transition in athermal disordered materials. Europhysics Letters 90:20005 (2010). http://dx.doi.org/10.1209/0295-5075/90/20005

Irani, E., Chaudhuri, P., and Heussinger, C. Impact of Attractive Interactions on the Rheology of Dense Athermal Particles. Physical Review Letters 112:188303 (2014). http://dx.doi.org/10.1103/PhysRevLett.112.188303

Irani, E., Chaudhuri, P., and Heussinger, C. Athermal rheology of weakly attractive soft particles. arXiv (2016). http://arxiv.org/abs/1605.01222

Israelachvili, J. Intermolecular and Surface Forces, 3rd Edition. Academic Press (2010). ISBN 978-0-12-375182-9.

Jaeger, H. M., Nagel, S. R., and Behringer, R. P. Granular solids, liquids, and gases. Reviews of Modern Physics 68:1259 (1996). http://dx.doi.org/10.1103/RevModPhys.68.1259

Jiang, W., Xuan, S., and Gong, X. The role of shear in the transition from continuous shear thickening to discontinuous shear thickening. Applied Physics Letters 106:151902 (2015). http://dx.doi.org/10.1063/1.4918344

Johnson, K. L. Contact Mechanics. Cambridge University Press (1985). ISBN 0521347963. http://dx.doi.org/10.1115/1.3261297

Jop, P., Forterre, Y., and Pouliquen, O. A constitutive law for dense granular flows. Nature 441:727 (2006). http://dx.doi.org/10.1038/nature04801

Kadanoff, L. Built upon sand: Theoretical ideas inspired by granular flows. Reviews of Modern Physics 71:435 (1999). http://dx.doi.org/10.1103/RevModPhys.71.435

Kantz, H. A robust method to estimate the maximal Lyapunov exponent of a time series. Physics Letters A 185:77 (1994). http://dx.doi.org/10.1016/0375-9601(94)90991-1

Kawasaki, T., Coslovich, D., Ikeda, A., and Berthier, L. Diverging viscosity and soft granular rheology in non-Brownian suspensions. Physical Review E 91:012203 (2015). http://dx.doi.org/10.1103/PhysRevE.91.012203 
Kranz, W. T., Sperl, M., and Zippelius, A. Glass transition for driven granular fluids. Physical Review Letters 104:225701 (2010). http://dx.doi.org/10.1103/PhysRevLett.104.225701

Kranz, W. T., Sperl, M., and Zippelius, A. Glass transition in driven granular fluids: a mode-coupling approach. Physical Review E 87:022207 (2013). http://dx.doi.org/10.1103/PhysRevE.87.022207

Landau, L. D. and Lifshitz, E. M. Theory of Elasticity, vol. 7. Pergamon Press (1970). ISBN 0080064655.

Lechenault, F., Dauchot, O., Biroli, G., and Bouchaud, J.-P. Critical scaling and heterogeneous superdiffusion across the jamming/rigidity transition of a granular glass. Europhysics Letters 83:46003 (2008). http://dx.doi.org/10.1209/0295-5075/83/46003

Lemaître, A. and Caroli, C. Rate-dependent avalanche size in athermally sheared amorphous solids. Physical Review Letters 103:065501 (2009). http://dx.doi.org/10.1103/PhysRevLett.103.065501

Lin, N. Y. C., Guy, B. M., Hermes, M., Ness, C., Sun, J., Poon, W. C. K., and Cohen, I. Hydrodynamic and Contact Contributions to Continuous Shear Thickening in Colloidal Suspensions. Physical Review Letters 115:228304 (2015). http://dx.doi.org/10.1103/PhysRevLett.115.228304

Liu, A. J. and Nagel, S. R. Nonlinear dynamics: Jamming is not just cool any more. Nature 396:21 (1998). http://dx.doi.org/10.1038/23819

Liu, H., Xie, X., and Xu, N. Finite Size Analysis of Zero-Temperature Jamming Transition under Applied Shear Stress by Minimizing a Thermodynamic-Like Potential. Physical Review Letters 112:145502 (2014). http://dx.doi.org/10.1103/PhysRevLett.112.145502

Lootens, D., Van Damme, H., and Hébraud, P. Giant stress fluctuations at the jamming transition. Physical Review Letters 90:178301 (2003). http://dx.doi.org/10.1103/PhysRevLett.90.178301

Lutz-Bueno, V., Kohlbrecher, J., and Fischer, P. Shear thickening, temporal shear oscillations, and degradation of dilute equimolar CTAB/NaSal wormlike solutions. Rheologica Acta 52:297 (2013). http://link.springer.com/10.1007/s00397-012-0672-4

Maloney, C. E. and Lemaître, A. Amorphous systems in athermal, quasistatic shear. Physical Review E 74:016118 (2006). http://dx.doi.org/10.1103/PhysRevE.74.016118 
Bibliography

Mari, R., Seto, R., Morris, J. F., and Denn, M. M. Shear thickening, frictionless and frictional rheologies in non-Brownian suspensions. Journal of Rheology 58:1693 (2014). http://dx.doi.org/10.1122/1.4890747

Mason, T. and Weitz, D. Optical measurements of frequency-dependent linear viscoelastic moduli of complex fluids. Physical Review Letters 74:1250 (1995). http://dx.doi.org/10.1103/PhysRevLett.74.1250

Michlmayr, G., Cohen, D., and Or, D. Sources and characteristics of acoustic emissions from mechanically stressed geologic granular media - A review. Earth-Science Reviews 112:97 (2012). http://dx.doi.org/10.1016/j.earscirev.2012.02.009

Midi, G. D. R. On dense granular flows. The European Physical Journal E 14:341 (2004). http://dx.doi.org/10.1140/epje/i2003-10153-0

Mindlin, R. D. and Deresiewicz, H. Elastic spheres in contact under varying oblique forces. American Society of Mechanical Engineers - Transactions Journal of Applied Mechanics 20:327 (1953).

Mullin, T. Granular materials. Mixing and de-mixing. Science (New York, N.Y.) 295:1851 (2002). http://dx.doi.org/10.1126/science.1070258

Ness, C. and Sun, J. Shear thickening regimes of dense non-Brownian suspensions. Soft Matter 12:914 (2015). http://dx.doi.org/10.1039/C5SM02326B

Ness, C. and Sun, J. Two-scale evolution during shear reversal in dense suspensions. Physical review. E 93:012604 (2016). http://dx.doi.org/10.1103/PhysRevE.93.012604

O'Hern, C. S., Langer, S. A., Liu, A. J., and Nagel, S. R. Random packings of frictionless particles. Physical Review Letters 88:075507 (2002). http://dx.doi.org/10.1103/PhysRevLett.88.075507

O’Hern, C. S., Silbert, L. E., Liu, A. J., and Nagel, S. R. Jamming at zero temperature and zero applied stress: The epitome of disorder. Physical Review E 68:011306 (2003). http://dx.doi.org/10.1103/PhysRevE.68.011306

Olmsted, P. D. Two-state shear diagrams for complex fluids in shear flow. Europhysics Letters 48:339 (1999). http://arxiv.org/abs/cond-mat/9909016 
Olmsted, P. D. Perspectives on shear banding in complex fluids. Rheologica Acta 47:283 (2008). http://dx.doi.org/10.1007/s00397-008-0260-9

Olsson, P. Dissipation and velocity distribution at the shear-driven jamming transition. Physical Review E 93:042614 (2016). http://dx.doi.org/10.1103/PhysRevE.93.042614

Olsson, P. and Teitel, S. Critical scaling of shear viscosity at the jamming transition. Physical Review Letters 99:178001 (2007). http://dx.doi.org/10.1103/PhysRevLett.99.178001

Olsson, P. and Teitel, S. Critical scaling of shearing rheology at the jamming transition of soft-core frictionless disks. Physical Review E 83:030302 (2011). http://dx.doi.org/10.1103/PhysRevE.83.030302

Olsson, P. and Teitel, S. Herschel-Bulkley shearing rheology near the athermal jamming transition. Physical Review Letters 109:108001 (2012). http://dx.doi.org/10.1103/PhysRevLett.109.108001

Otsuki, M. and Hayakawa, H. Universal scaling for the jamming transition. Progress of Theoretical Physics 121:9 (2008). http://dx.doi.org/10.1143/PTP.121.647

Otsuki, M. and Hayakawa, H. Critical behaviors of sheared frictionless granular materials near the jamming transition. Physical Review E 80:011308 (2009). http://dx.doi.org/10.1103/PhysRevE.80.011308

Otsuki, M. and Hayakawa, H. Critical scaling near jamming transition for frictional granular particles. Physical Review E 83:051301 (2011). http://dx.doi.org/10.1103/PhysRevE.83.051301

Otsuki, M., Hayakawa, H., and Luding, S. Behavior of Pressure and Viscosity at High Densities for Two-Dimensional Hard and Soft Granular Materials. Progress of Theoretical Physics Supplement 184:110 (2010). http://dx.doi.org/10.1143/PTPS.184.110

Pan, Z., de Cagny, H., Weber, B., and Bonn, D. S-shaped flow curves of shear thickening suspensions: direct observation of frictional rheology. Physical Review E 92:032202 (2015). http://dx.doi.org/10.1103/PhysRevE.92.032202

Picano, F., Breugem, W. P., Mitra, D., and Brandt, L. Shear thickening in nonBrownian suspensions: An excluded volume effect. Physical Review Letters 111:098302 (2013). http://dx.doi.org/10.1103/PhysRevLett.111.098302 
Bibliography

Pouliquen, O. Velocity correlations in dense granular flows. Physical Review Letters 93:248001 (2004).

http://dx.doi.org/10.1103/PhysRevLett.93.248001

Puertas, A. M. and Voigtmann, T. Microrheology of colloidal systems. Journal of physics: Condensed matter 26:243101 (2014). http://dx.doi.org/10.1088/0953-8984/26/24/243101

Reichhardt, C. and Reichhardt, C. J. O. Aspects of jamming in two-dimensional athermal frictionless systems. Soft matter 10:2932 (2014). http://dx.doi.org/10.1039/c3sm53154f

Rosenstein, M. T., Collins, J. J., and De Luca, C. J. A practical method for calculating largest Lyapunov exponents from small data sets. Physica D: Nonlinear Phenomena 65:117 (1993). http://dx.doi.org/10.1016/0167-2789(93)90009-P

Royer, J. R., Blair, D. L., and Hudson, S. D. Rheological Signature of Frictional Interactions in Shear Thickening Suspensions. Physical Review Letters 116:188301 (2016). http://dx.doi.org/10.1103/PhysRevLett.116.188301

Sano, M. and Sawada, Y. Measurement of the lyapunov spectrum from a chaotic time series. Physical Review Letters 55:1082 (1985). http://dx.doi.org/10.1103/PhysRevLett.55.1082

Schäfer, J., Dippel, S., and Wolf, D. E. Force Schemes in Simulations of Granular Materials. Journal de Physique I 6:5 (1996). http://dx.doi.org/10.1051/jp1:1996129

Schall, P. and van Hecke, M. Shear Bands in Matter with Granularity. Annual Review of Fluid Mechanics 42:67 (2010). http://dx.doi.org/10.1146/annurev-fluid-121108-145544

Schwager, T. and Pöschel, T. Coefficient of restitution and linear-dashpot model revisited. Granular Matter 9:465 (2007). http://dx.doi.org/10.1007/s10035-007-0065-z

Seto, R., Mari, R., Morris, J. F., and Denn, M. M. Discontinuous shear thickening of frictional hard-sphere suspensions. Physical Review Letters 111:218301 (2013). http://dx.doi.org/10.1103/PhysRevLett.111.218301

Silbert, L. E. Temporally heterogeneous dynamics in granular flows. Physical Review Letters 94:098002 (2005). http://dx.doi.org/10.1103/PhysRevLett.94.098002 
Silbert, L. E. Jamming of frictional spheres and random loose packing. Soft Matter 6:2918 (2010). http://dx.doi.org/10.1039/c001973a

Silbert, L. E., Ertaş, D., Grest, G. S., Halsey, T. C., Levine, D., and Plimpton, S. J. Granular flow down an inclined plane: Bagnold scaling and rheology. Physical Review E 64:051302 (2001). http://dx.doi.org/10.1103/PhysRevE.64.051302

Small, M., Walker, D. M., Tordesillas, A., and Tse, C. K. Characterizing chaotic dynamics from simulations of large strain behavior of a granular material under biaxial compression. Chaos (Woodbury, N.Y.) 23:013113 (2013). http://dx.doi.org/10.1063/1.4790833

Sollich, P., Lequeux, F., Hébraud, P., and Cates, M. E. Rheology of Soft Glassy Materials. Physical Review Letters 78:2020 (1997). http://dx.doi.org/10.1103/PhysRevLett.78.2020

Song, C., Wang, P., and Makse, H. A. A phase diagram for jammed matter. Nature 453:629 (2008). http://dx.doi.org/10.1038/nature06981

Sperl, M., Kranz, W. T., and Zippelius, A. Single-particle dynamics in dense granular fluids under driving. Europhysics Letters 98:28001 (2012). http://dx.doi.org/10.1209/0295-5075/98/28001

Squires, T. M. and Mason, T. G. Fluid Mechanics of Microrheology. Annual Review of Fluid Mechanics 42:413 (2010). http://dx.doi.org/10.1146/annurev-fluid-121108-145608

Sriram, I., Furst, E. M., DePuit, R. J., and Squires, T. M. Small amplitude active oscillatory microrheology of a colloidal suspension. Journal of Rheology 53:357 (2009). http://dx.doi.org/10.1122/1.3058438

Sutherland, W. A dynamical theory of diffusion for non-electrolytes and the molecular mass of albumin. Philosophical Magazine 9:781 (1905). http://dx.doi.org/10.1080/14786440509463331

Tewari, S., Tithi, B., Ferguson, A., and Chakraborty, B. Growing length scale in gravity-driven dense granular flow. Physical Review E 79:011303 (2009). http://dx.doi.org/10.1103/PhysRevE.79.011303

Vågberg, D., Olsson, P., and Teitel, S. Glassiness, rigidity, and jamming of frictionless soft core disks. Physical Review E 83:031307 (2011). http://dx.doi.org/10.1103/PhysRevE.83.031307 
Bibliography

Vågberg, D., Olsson, P., and Teitel, S. Dissipation and Rheology of Sheared SoftCore Frictionless Disks Below Jamming. Physical Review Letters 112:208303 (2014). http://dx.doi.org/10.1103/PhysRevLett.112.208303

Varnik, F. and Raabe, D. Profile blunting and flow blockage in a yield-stress fluid: A molecular dynamics study. Physical Review E 77:011504 (2008). http://dx.doi.org/10.1103/PhysRevE.77.011504

Volfson, D., Tsimring, L. S., and Aranson, I. S. Partially fluidized shear granular flows: continuum theory and molecular dynamics simulations. Physical Review E 68:021301 (2003). http://dx.doi.org/10.1103/PhysRevE.68.021301

Wagner, N. J. and Brady, J. F. Shear thickening in colloidal dispersions. Physics Today 62:27 (2009). http://dx.doi.org/10.1063/1.3248476

Wang, T., Grob, M., Zippelius, A., and Sperl, M. Active microrheology of driven granular particles. Physical Review E 89:042209 (2014). http://dx.doi.org/10.1103/PhysRevE.89.042209

Wilson, L. G., Harrison, A. W., Schofield, A. B., Arlt, J., and Poon, W. C. K. Passive and active microrheology of hard-sphere colloids. The journal of physical chemistry. B 113:3806 (2009).

http://dx.doi.org/10.1021/jp8079028

Wyart, M. and Cates, M. E. Discontinuous shear thickening without inertia in dense non-brownian suspensions. Physical Review Letters 112:098302 (2014). http://dx.doi.org/10.1103/PhysRevLett.112.098302

Zhang, L., Zheng, J., and Zhang, J. On the route to shear jamming, are fragile states real? arXiv (2015). http://arxiv.org/abs/1505.00300 


\section{Author contribution}

\section{Jamming of frictional particles: A nonequilibium first-order phase transition}

Grob, M., Heussinger, C., and Zippelius, A.

Jamming of frictional particles: a nonequilibrium first-order phase transition.

Physical Review E 89:050201 (2014).

http://dx.doi.org/10.1103/PhysRevE.89.050201

The article covers molecular dynamics studies of frictional grains and the analysis of a phenomenological model. I developed the code of the simulation, conducted all the numerical simulations, analyzed the model, and created all the figures and the supplemental material. The study was designed by me, Claus Heussinger, and Annette Zippelius. The article was mainly written by Annette Zippelius.

\section{Rheological chaos of frictional grains}

Grob, M., Zippelius, A., and Heussinger, C.

Rheological chaos of frictional grains.

Physical Review E 93:030901 (2016).

http://dx.doi.org/10.1103/PhysRevE.93.030901

The article covers molecular dynamics studies of frictional grains and the analysis of a hydrodynamic model. I developed the code of the simulation, conducted all the numerical simulations, derived and analyzed the model, and created all the figures and the supplemental material. The study was designed by me, Claus Heussinger, and Annette Zippelius. The articles was written by me, Claus Heussinger, and Annette Zippelius. 
Author contribution

\section{Unsteady rheology and heterogeneous flow of dry frictional grains}

The manuscript covers molecular dynamics studies of frictional grains. I developed the code of the simulation, conducted all the numerical simulations, and created all the figures. The study was designed by me, Claus Heussinger, and Annette Zippelius. The manuscript was written by me with revisions from Claus Heussinger and Annette Zippelius.

\section{Active microrheology of driven granular particles}

Wang, T., Grob, M., Zippelius, A., and Sperl, M.

Active microrheology of driven granular particles.

Physical Review E 89:042209 (2014).

http://dx.doi.org/10.1103/PhysRevE.89.042209

The article covers a study that was designed by Ting Wang and Matthias Sperl. I contributed the molecular dynamics results. Ting Wang and Matthias Sperl wrote the article with support by me and revisions from Annette Zippelius. 


\section{Acknowledgments}

Meiner Betreuerin Prof. Dr. Annette Zippelius möchte ich einen tiefen Ausdruck von Dank entgegenbringen. Sie hat mich während der letzten Jahre stets gefördert und gefordert, viele interessante Dienstreisen wahrnehmen lassen und war stets bereit Ratschläge und Meinungen in sämtlichen Belangen zu äußern. Herrn Dr. Claus Heussinger, ebenfalls mein Betreuer, gilt auch großer Dank. Er hat produktive Denkanstöße gegeben und war für inhaltliche und auch technische Probleme immer ein hilfreicher Ansprechpartner. Prof. Dr. Reiner Kree danke ich für die Übernahme des Zweitgutachtens.

Weiter möchte ich den Mitarbeitern der Gruppen Zippelius und Heussinger danken. Allen voran Elham und Ehsan, Till, Florian, Ronny, Andrea und Iraj.

Special thanks go to the group of Prof. R.P. Behringer at Duke University, Durham, NC, USA. He and his (former) group members Jonathan Barès and Dong Wang gave me hospitality in the probably most-established laboratory for granular materials.

Großer Dank gilt auch der Verwaltung des Instituts. Durch Frau Schubert und Frau Glormann und meine Rolle als Gleichstellungsbeauftragter habe ich viel über administrative Vorgänge im Institut lernen können.

Technische Unterstützung kam vor allem von Dr. Jürgen Holm und Thomas Köhler, die die Hard- und Software im Institut pflegen. Die vielen CUDA-Rechner wären ohne Andre Galuschko nicht ins Institut gekommen.

Weiter möchte ich mich bei Freunden und Familie bedanken. Allen voran geht hier Knut Heidemann, mit dem ich seit dem Studium freundschaftliche und fachliche Kontakte pflege. Besonders bemerkenswert ist sein Engagement vor der Fertigstellung meiner Dissertation. Meiner lieben Friederike möchte ich danken, dass sie des Öfteren mit lieben Worten und Taten für Ausgleich zur Arbeit sorgen konnte. Japser Cirkel möchte ich für weiteres Korrekturlesen danken. Elterliche Unterstützung hätte ich nicht missen wollen. 



\section{List of Publications}

\section{Publications related to this thesis}

- Grob, M., Zippelius, A., and Heussinger, C.

Rheological chaos of frictional grains.

Physical Review E 93:030901 (2016).

http://dx.doi.org/10.1103/PhysRevE.93.030901

- Grob, M., Heussinger, C., and Zippelius, A.

Jamming of frictional particles: a nonequilibrium first-order phase transition.

Physical Review E 89:050201 (2014).

http://dx.doi.org/10.1103/PhysRevE.89.050201

- Wang, T., Grob, M., Zippelius, A., and Sperl, M.

Active microrheology of driven granular particles.

Physical Review E 89:042209 (2014).

http://dx.doi.org/10.1103/PhysRevE.89.042209

\section{Further publications}

- Fiege, A., Grob, M., and Zippelius, A.

Dynamics of an intruder in dense granular fluids.

Granular Matter 14:247 (2012).

http://dx.doi.org/10.1007/s10035-011-0309-9 